\title{
Finite size effects near the onset of the oscillatory instability
}

\author{
Carlos Martel and José M Vega \\ ETSI Aeronáuticos, Universidad Politéenica de Madrid, 28040-Madrid. Spain
}

\begin{abstract}
A system of two complex Ginzhurg-Landau equations is considered that applies at the onset of the oseillatory instability in spatial domains whose size is large (but finite) in one direction; the dependent variables are the slowly modulated complex amplitudes of two counterpropagating wavetrains. In order to obtain a well posed problem, four boundary conditions must be imposed at the boundaries. Two of them were already known, and the other two are first derived in this paper. In the generic case when the group velocity is of order unity, the resulting problem has terns that are not of the same order of magnitude. This fact allows us to consider two distinguished linits and to derive two associated (simpler) sub-models, that are briefly discussed. Our results predict quite a rich variety of complex dynamics that is due to both the modulational instability and finite size effects.
\end{abstract}

AMS classification scheme numbers: $35 \mathrm{~B} 25,35 \mathrm{~B} 32,35 \mathrm{Q} 35,76 \mathrm{E} 30$

\section{Introduction}

The oscillatory instability is one of the few generic universal ways [1] in which nearly uniform steady states of physical systems in large spatial domains (i.e. large compared with the appropriate inherent characteristic length) may lose stability. Experimental results in fluid systems showed that a large variety of complex spatio-temporal structures appear near the onset of that instability. These experiments were concerned with binary fluid convection [2-5], isothermal double diffusive convection [6], a secondary instability of rolls in pure Rayleigh-Benard convection [7-9], spiral waves in the Taylor-Couette system [10.11], transversal waves in thermocapillary flows [12-14]. electrolydrodynamic convection in nematic liquid crystals [2, 15-18] and several combustion systems [19-23]. A remarkable feature is that these structures were essentially one-dimensional for a wide range of the parameters; this was sometimes (but not always) due to anisotropy properties of the underlying plysical problem.

A systematic weakly nonlinear analysis near the onset of this instability has been undertaken in recent years. If the governing equations are invariant under spatial translations and reflection. then the marginally stable modes associated with this instability consist of a pair of counterpropagating wavetrains. The amplitude equations accounting for weakly nonlinear interaction of the counterpropagating waves were first derived by Coullet $e t$ al [24]. who also obtained some significant particular time-dependent solutions and analysed their stability (see also [25,26]). These amplitude equations are the following coupled 
complex Ginzburg-Landau equations

$$
\begin{aligned}
& A_{t}=c A_{x x}+\left(b_{0}+\mu b_{1}\right) A_{x}+A\left[\left(d_{0}+\mu d_{1}\right) \mu+e_{1}|A|^{2}+e_{2}|B|^{2}\right] \\
& B_{t}=c B_{x x}-\left(b_{0}+\mu b_{1}\right) B_{x}+B\left[\left(d_{0}+\mu d_{1}\right) \mu+e_{1}|B|^{2}+e_{2}|A|^{2}\right] .
\end{aligned}
$$

Here $A$ and $B$ are the complex amplitudes of the two wavetrains, and depend weakly on the space and time variables $x$ and $t$. The control parameter $\mu$ is real and such that $|\mu| \ll 1$. The group velocity $b_{0}$ is real while the remaining coefficients, $b_{1}, c, d_{0} . d_{1}, e_{1}$ and $e_{2}$, are complex in general. Notice that we are considering a second approximation of the coefficients of $A_{x}, B_{x}, \mu A$ and $\mu B$; this is required by the asymptotic consistency of the underlying perturbation analysis, as will be seen below. Also the weakly nonlinear level of this approach requires (essentially) that

$$
\begin{aligned}
& |\mu| \ll 1 \quad\left|A_{t}\right| \ll|A| \ll 1 \quad\left|B_{t}\right| \ll|B| \ll 1 \\
& \left|A_{x, x}\right| \ll\left|A_{x}\right| \ll|A| \quad\left|B_{x, x}\right| \ll\left|B_{x}\right| \ll|B|
\end{aligned}
$$

while $\left|b_{i}\right|,\left|b_{1}\right|_{.}|c| .\left|d_{0}\right|,\left|d_{1}\right|,\left|e_{1}\right|$ and $\left|e_{2}\right|$ remain of order unity. In particular, the group velocity $b_{0}$ is a bounded away from zero except in the neighbourhood of a certain codimension-2 manifold of the parameter space of the underlying physical problem. and this fact will be essential in this paper. Let us mention here that the amplitude equations (1.1), (1.2), with the coefficients, $c, e_{1}$ and $e_{2}$ purely imaginary (then (1.1) and (1.2) are nonlinear Schrödinger equations) appear also when studying wave propagation in conservative systems. invariant under reflection symmetry: two examples among several are: capillary waves (see [27-29] for recent weakly nonlinear analyses through the amplitude equations) and electromagnetic waves in optical fibres [30].

If the spatial domain of the plysical system leading to (1.1) and (1.2) is large but finite, then (1.1) and (1.2) must be considered in a finite interval $-L / 2<x<L / 2$. with $L \gg 1$, and four boundary conditions must be imposed at the ends of the interval, $x=-L / 2$ and $L / 2$. Two of them were first introduced by Cross [31-34]:

$$
B=r A \quad \text { at } x=-L / 2 \quad A=r B \quad \text { at } x=L / 2
$$

and account for linear reflection of the wavetrains at the ends of the interval. The (complex in general) reflection coefficient $r$ may be calculated from matching conditions between the solution of the (linearized) problem governing the underlying physical system in two boundary layers where $|x \pm L / 2| \sim 1$, and the solution in the bulk, where $|x \pm L / 2| \gg$ I (see [34]).

The other two boundary conditions will be derived in section 2 in a particular case. and in appendix $\mathrm{A}$ in a more general setting, by higher order matching between the solution in the bulk and in the boundary layers near $x=-L / 2$ and $L / 2$. These new conditions are

$$
\begin{array}{ll}
b_{0}\left(B_{x}+r A_{x}\right)=\left(e_{1}-e_{2}\right) r\left(|r|^{2}-1\right) A|A|^{2} & \text { at } x=-L / 2 \\
b_{0}\left(A_{x}+r B_{x}\right)=\left(e_{2}-e_{1}\right) r\left(|r|^{2}-1\right) B|B|^{2} & \text { at } x=L / 2 .
\end{array}
$$

Notice that these conditions are nonlinear if $\left(e_{1}-e_{2}\right) r\left(|r|^{2}-1\right)$ is non-zero, as may be assumed to be the case generically; nevertheless, the (nongeneric without further restrictions) case $|r|=1$, corresponding to perfectly reflecting boundaries, deserves some attention (this will be paid below, for example in section 4). Also, as will be seen in section 5 , any other pair of boundary conditions (different from (1.5) and (1.6)) would lead to inconsistencies in the weakly nonlinear description of the instability. 
If the spatial domain has no boundaries (e.g. if it is an annulus, as in the experiment in [35]) then conditions (1.4) and (1.5) must be replaced by the new ones:

$A(x+L, t)=\mathrm{e}^{\mathrm{it} t} A(x, t) \quad B(x+L, t)=\mathrm{e}^{-t \nu} B(x, t) \quad$ for all $x$

that account for spatial periodicity. The length of the domain. $L \gg 1$, and the phase shift, $v$, are given constants. The resulting problem, (1.1), (1.2). (1.4). will be discussed in section 6 .

The assumption $\left|b_{0}\right|^{-1}=\mathrm{O}(1)$ is essential in the derivation of $(1.5),(1.6)$. On the other hand, this assumption means that, when conveniently re-scaled (see section 3 ), equations (1.1) and (1.2) contain terms that are not of the same order. This fact may be seen as a difficulty (see [34]). In fact, we will need to consider two distinguished limits in section 3 (depending on the relative values of the small parameters $L^{-1}$ and $\mu$ ) which lead to two submodels of (1.1), (1.2). (1.4)-(1.6) that are essentially different. But, although the derivation and analysis of these submodels require some subtleties. they are significantly simpler than (1.1). (1.2), (1.4)-(1.6); in particular, they are more amenable to purely analytical treatment. Notice that nothing is wrong with considering equations such as (1.1), (1.2), that contain terms not of the same order, as long as the consistency conditions (1.3) are not violated.

The basic steady state, $A \equiv B \equiv 0$, becomes unstable as $\mu>\mu_{c}=-2 L^{-1} b_{0}\left(d_{0}+\right.$ $\left.\bar{d}_{0}\right)^{-1} \log |r|+\mathrm{O}\left(L^{-2}\right)$, where overbars stand hereafter for the complex conjugate; this shift in the instability limit is due to the presence of non-perfectly reflecting boundaries, as was first pointed out by Cross [31] to explain some experimental results. Then, two distinguished limits must be considered when analysing (1.1), (1.2), (1.4)-(1.6) as $L \rightarrow \infty$ and $\mu \rightarrow 0$,

$$
\begin{aligned}
\left|\mu-\mu_{c}\right| & \sim L^{-2} & |A| & \sim|B| \sim L^{-1} \\
\left|\mu-\mu_{c}\right| & \sim L^{-1} & |A| & \sim|B| \sim L^{-1 / 2} .
\end{aligned}
$$

The limit (1.7) will be considered in section 4 , where we shall need to consider one spatial scale, $x \sim L$ and two time scales, $t \sim L$ and $t \sim L^{2}$. Then we shall obtain a complex Ginzburg-Landau equation with a non-local, spatially averaged term, for the evolution of the amplitudes in the slower time scale. Non-local equations of this type seem to have first been derived systematically, in the analysis of counterpropagating pairs of waves, by Chikwendu and Kevorkian [36]. More recently these equations were obtained in the analysis of the oscillatory instability of 1D spatially constant steady states [37] and 2D planar wavefronts [38], by means of two-timing scales methods (see also [39] for a rigorous derivation and $[30,40,41]$ for some further analysis concerning this equation); in fact, in [38] the equations appeared, as a particular limit, in a weakly nonlinear stability analysis of a 2D wavefront of a reaction-diffusion system arising in combustion theory. But the derivation in [37-39] was made under the assumption of perfectly reflecting boundaries. i.e. $|r|=1$. while here we allow arbitrary values of the reflection coefficient. Also. we shall obtain a somewhat more general equation that exhibits some essentially new behaviour, as will be explained at the end of section 4 . A more complete analysis of the complex averaged Ginzburg-Landau equation is given elsewhere [41.42].

In the limit (1.8) we are led to consider two spatial scales, $x \sim L^{1 / 2}$ and $x \sim L$, and two time scales, $t \sim L^{1 / 2}$ and $t \sim L$. In section 5 we shall analyse the stability of the solutions depending only on the larger space and time scales under perturbations depending on the shorter spatial scale. When the latter does not come into play, the evolution of the amplitudes is governed by a pair of real nonlinear wave equations (RNWE) that depend only on the size of the reflection coefficient $|r|$. the ratio of the real parts of $e_{1}$ and $e_{2}$, and the re-scaled control parameter, $\mu L$. For a fairly complete analysis of these RNWE, 
see $[41,43,44]$. Let us mention here that the RNWE explain quantitatively (with surprising accuracy) experimental results $[8,9]$ in pure Rayleigh-Benard convection (see $[41,44]$ ).

Here we are not considering the limit $b_{0} \rightarrow 0$. In this limit Cross [3 I-34] introduced (by phenomenological arguments) some linear boundary conditions with adjustable coefficients. By applying these conditions to (1.1). (1.2) some experimental observations may be explained qualitatively.

Finally, let us mention that a different approach [45-50]. based on an averaging procedure, leads to a pair of ODEs to describe the evolution of the amplitudes; the effect of the end-walls may be somehow taken into account in this model. Although it seems obvious that this approach cannot give the complete and detailed spatio-temporal picture (as we expect our results do) it has the advantage of providing a much simpler model, without ignoring completely the qualitative effect of the main physical mechanisms involved.

\section{The complete set of boundary conditions}

In this section and in appendix A we shall obtain the boundary conditions (1.4)-(1.6) and show that they apply to a large variety of physical problems. For the sake of clarity we first obtain those conditions for one of the simplest problems that may exhibit the oscillatory instability, and relegate to appendix A the derivation in a more general setting. We consider the $1 \mathrm{D}$ reaction-diffusion system

$$
\begin{aligned}
& u_{i}=D u_{x x}+f(u, \mu) \quad \text { in }-L / 2<x<L / 2 \\
& C u \pm E u_{x}=W \quad \text { at } x= \pm L / 2
\end{aligned}
$$

where $u \in \mathbb{R}^{N}(N>1), C, D$ and $E$ are constant $N \times N$ matrices, $\mu$ is the control parameter, $f$ is the nonlinear reaction term, and $W$ is a vector of $\mathbb{R}^{N}$.

We shall consider the limit

$$
|\mu| \ll 1 \quad L \gg 1
$$

and assume that, for all sufficiently small values of $\mu,(2.1),(2.2)$ has a steady state that is spatially uniform in first approximation except perhaps in two boundary layers near $x= \pm L / 2$ (see figure 1). If (without loss of generality) the uniform value of $u$ in the bulk is assumed to vanish, then we have

$$
f(0, \mu) \equiv 0
$$

and the nonlinearity $f$ may be expanded around $(u, \mu)=(0,0)$ as

$$
f(u, \mu)=\left(F_{1}+\mu F_{2}+\mu^{2} F_{3}\right) u+\mathcal{B}(u, u)+\mathcal{C}(u, u, u)+\mathrm{O}\left(|\mu|^{3}+|\mu u|+|u|^{3}\right)|u|
$$

as $u \rightarrow 0$ and $\mu \rightarrow 0$. where $F_{1}=f_{u}(0,0), F_{2}=f_{u \mu}(0,0)$ and $F_{3}=f_{u \mu \mu}(0,0) / 2$ are $N \times N$ matrices, while $\mathcal{B}=f_{u t}(0,0) / 2$ and $\mathcal{C}=f_{u t u}(0,0) / 6$ are the multilinear symmetric

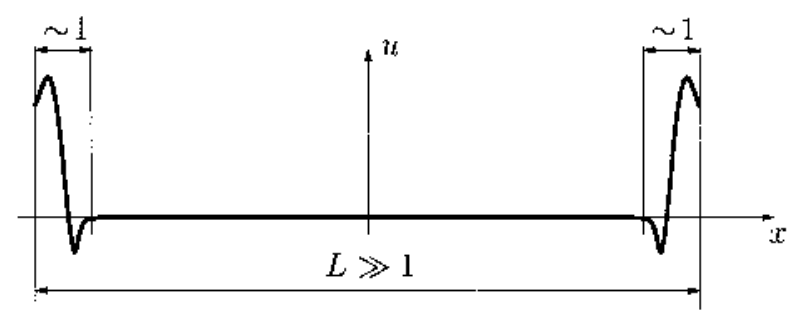

Figure 1. Sketch of the basic steady state. 
operators, associated with the second- and third-order terms in the Taylor expansion of $f(u, 0)$. Some additional assumptions will be made below when needed.

We now consider the solution of (2.1), (2.2) both in the bulk and in the boundary layers and apply matching conditions to obtain the appropriate boundary conditions for the solution in the bulk: the ideas below are the standard ones in the method of matched astmptotic expansions [51.52] (see also [53] for a discussion on the application of the method to bifurcation problems in large domains). A somewhat close look at the weakly nonlinear description of the solution will be necessary in order to derive the boundary conditions (1.4)-(1.6). Notice that we are not introducing at this stage re-scalings relating the several small variables and parameters ( $A, B$ and their derivatives, $|\mu|$ and $L^{-1}$ ) because we intend to obtain general boundary conditions (not depending on particular re-scalings).

\subsection{The solution of (2.1), (2.2) in the bulk, $-L / 2<x<L / 2,|x \pm L / 2| \gg 1$}

We assume that the steady state $u=0$ exhibits the oscillatory instability at $\mu=0$. More precisely, if the ansatz

$$
u=U \exp (\Omega 2 t+\mathrm{i} k x)+\text { c.c. }+\mathrm{o}(|U|) \quad \text { with }|U| \ll 1
$$

is inserted into (2.1). the resulting linearized eigenvalue problem possesses two pairs of algebraically simple, complex conjugate eigenvalues. $\Omega_{ \pm}$and $\bar{\Omega}_{ \pm}$(hereafter. overbars and c.c. stand for the complex conjugate), such that

$\Omega_{ \pm}=\mathrm{i} \omega \pm \mathrm{i}\left(b_{0}+\mu b_{1}\right)\left(k \mp k_{0}\right)-c\left(k \mp k_{0}\right)^{2}+\left(d_{0}+\mu d_{1}\right) \mu+\mathrm{O}\left(|\mu|+\left|k \mp k_{0}\right|\right)^{3}$

as $k \rightarrow \pm k_{0}$ and $\mu \rightarrow 0$, where $\omega>0, k_{0}>0$ and $b_{0} \neq 0$ are real constants. while $b_{1}$, $c . d_{0}$ and $d_{1}$ are complex constants such that the real parts of $c$ and $d_{0}$ are strictly positive (see figure 2): the remaining part of the spectnum is assumed to have a strictly negative real part, and to be at a non-zero distance from the imaginary axis. In fact. we only need to assume that the dispersion relation has one of the branches (2.5): the other one readily exists because (2.1) is invariant under spatial reflection, $x \rightarrow-x$. The coefficients $b_{0}, b_{1}$. $c, d_{0}$ and $d_{1}$ may be calculated by expanding the eigenvector associated with $\Omega_{+}$as

$U=U_{0}+\left(k-k_{0}\right) U_{1}+\left(k-k_{0}\right)^{2} U_{2}+\mu U_{3}+\mu\left(k-k_{0}\right) U_{4}+\mu^{2} U_{5}+\mathrm{O}\left(|\mu|+\left|k-k_{0}\right|\right)^{3}$

and inserting this expansion, (2.4) and (2.5) into the linearized equation

$$
\left[f_{u}(0, \mu)-k^{2} D-\Omega_{+} I\right] U=0
$$

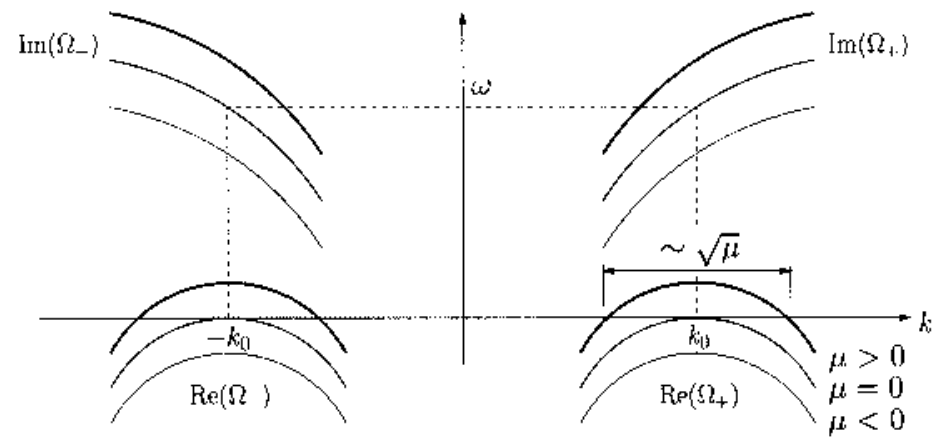

Figure 2. Dispersion relation near criticality. 
to obtain

$$
\begin{aligned}
& \left(F_{1}-k_{0}^{2} D-\mathrm{i} \omega I\right) U_{0}=0 \quad U_{0} \neq 0 \\
& \left(F_{1}-k_{0}^{2} D-\mathrm{i} \omega I\right) U_{1}=\left(2 k_{0} D+\mathrm{i} b_{0} I\right) U_{0} \\
& \left(F_{1}-k_{0}^{2} D-\mathrm{i} \omega I\right) U_{2}=(D-c I) U_{0}+\left(2 k_{0} D+\mathrm{i} b_{0} I\right) U_{1} \\
& \left(F_{1}-k_{0}^{2} D-\mathrm{i} \omega I\right) U_{3}=\left(d_{0} I-F_{2}\right) U_{0} \\
& \left(F_{1}-k_{0}^{2} D-\mathbf{i} \omega I\right) U_{4}=\mathrm{i} b_{1} U_{0}+\left(d_{0} I-F_{2}\right) U_{1}+\left(\mathrm{i} b_{0} I+2 k_{0} D\right) U_{3} \\
& \left(F_{1}-k_{0}^{2} D-\mathrm{i} \omega I\right) U_{5}=\left(d_{1} I-F_{3}\right) U_{0}+\left(d_{0} I-F_{2}\right) U_{3}
\end{aligned}
$$

where $I$ is the $N \times N$ unit matrix. Then, the solvability conditions of (2.7)-(2.11) yield

$$
b_{0}=2 \mathrm{i} k_{j} \bar{U}_{0}^{* T} D U_{0} \quad c=\bar{U}_{0}^{* T} D U_{0}+\bar{U}_{0}^{* T}\left(2 k_{0} D+\mathbf{i} b_{0} I\right) U_{1} \quad d_{0}=\bar{U}_{0}^{* T} F_{2} U_{0}
$$

$b_{1}=\bar{U}_{0}^{* T}\left(\mathbf{i} d_{0} I-\mathbf{i} F_{2}\right) U_{1}+\bar{U}_{0}^{* T}\left(2 \mathrm{i} k_{0} D-b_{0} I\right) U_{3} \quad d_{1}=\bar{U}_{0}^{* T} F_{3} U_{0}+\bar{U}_{0}^{* T}\left(F_{2}-d_{0} I\right) U_{3}$

where the superscript $T$ stands for the transpose and $U_{u}^{*}$ is a normalized eigenvector of the adjoint linearized problem

$$
\left(F_{1}-k_{0}^{2} D+\mathbf{i} \omega I\right)^{T} U_{0}^{*}=0 \quad \bar{U}_{0}^{* T} U_{0}=1 .
$$

Notice that the constants, $b_{0}, b_{1}, c, d_{0}$ and $d_{1}$ do not depend on the particular solutions of (2.6)-(2.11) that are selected, and that (recall that the eigenvalue $\mathrm{i} \omega$ of (2.6) is algebraically simple) the nontrivial solutions of the first equation in (2.14) are such that $\bar{U}_{0}^{* T} U_{0} \neq 0$.

The weakly nonlinear evolution of $u$ is obtained by considering the ansatz

$$
u=U_{0}\left(A \mathrm{e}^{\mathrm{i} \omega t+\mathrm{i} k_{0} x}+B \mathrm{e}^{\mathrm{i} \omega t-\mathrm{i} k_{0} x}\right)+\text { c.c. }+ \text { higher order terms }
$$

where the complex variables $A$ and $B$ are small and depend weakly on $x$ and $t$. Notice that $A$ and $B$ are the complex amplitudes of two wavetrains that are travelling (with phase velocities $\mp \omega / k_{0}$ ) to the left and to the right, respectively. The evolution equations for $A$ and $B$ may be found. for example, by (a multiple scales analysis consisting of) introducing (2.15) into (2.1) and requiring $u$ to be bounded (i.e. eliminating secular terms) in the time scale $t \sim 1$ (see [51] or [54]). When applying the method it is useful to take into account that non-resonant forcing terms (i.e. those terms depending on the fast space and time variables as $\exp \left(i m \omega t+\mathrm{i} n k_{0} x\right)$. with $m^{2} \neq 1$ or $n^{2} \neq 1$ ) do not have a contribution on secular terms because they provide a bounded evolution in the fast time scale, depending on the fast space and time variables precisely as $\exp \left(i m \omega t+\mathrm{i} n k_{0} x\right)$; resonant forcing terms instead provide an unbounded evolution in the fast time scale, depending on the fast space and time variables as $t \exp \left( \pm \mathrm{i} \omega t \pm \mathrm{i} k_{0} x\right)$ unless two (one for each counterpropagating wavetrain) appropriate solvability conditions are satisfied at each asymptotic order. When collecting those solvability conditions the following evolution equations result for the complex amplitudes $A$ and $B$

$$
\begin{aligned}
& A_{t}=\left(b_{0}+\mu b_{1}\right) A_{x}+c A_{x x}+A\left(d_{0} \mu+d_{1} \mu^{2}+e_{1}|A|^{2}+e_{2}|B|^{2}\right)+\text { HORTI } \\
& B_{f}=-\left(b_{0}+\mu b_{1}\right) B_{x}+c B_{x x}+B\left(d_{0} \mu+d_{1} \mu^{2}+e_{1}|B|^{2}+e_{2}|A|^{2}\right)+\text { HORT2 }
\end{aligned}
$$

while $u$ is seen to be given by

$$
\begin{aligned}
u=\left[\left(A U_{0}-\right.\right. & \mathrm{i} A_{x}\left(U_{1}+\mu U_{4}\right)-A_{x x} U_{2}+\mu A\left(U_{3}+\mu U_{5}\right)+A|A|^{2} U_{8} \\
& \left.\left.+A|B|^{2} U_{9}+\mathrm{HORT} 1\right) \mathrm{e}^{\mathrm{i} \omega t+i k_{0} x}+\mathrm{c.c} .\right] \\
& +\left[\left(B U_{0}+\mathrm{i} B_{x}\left(U_{1}+\mu U_{4}\right)-B_{x x} U_{2}+\mu B\left(U_{3}+\mu U_{5}\right)+B|B|^{2} U_{8}\right.\right.
\end{aligned}
$$




$$
\begin{aligned}
& \left.\left.+B|A|^{2} U_{9}+\text { HORT2 }\right) \mathrm{e}^{\mathrm{i} \omega t-i k_{0} x}+\text { c.c. }\right] \\
& +\left[\left(A^{2} \mathrm{e}^{2 i k_{0} x}+B^{2} \mathrm{e}^{-2 i k_{0} x}\right) U_{6} \mathrm{e}^{2 \mathrm{i} \omega t}+A B W_{1} \mathrm{e}^{2 \mathrm{i} \omega t}+A \bar{B} W_{2} \mathrm{e}^{2 i k_{0} x}+\text { c.c. }\right] \\
& +\left(|A|^{2}+|B|^{2}\right) U_{7}+\text { HONRT }
\end{aligned}
$$

where HONRT (higher order non-resonant terms) stands for non-resonant terms that are of higher order than those displayed. while HORTI and HORT2 (higher order resonant terms) stand for

$$
\text { HORTI }=\mathrm{O}\left[\left(\mu^{2}+|A|^{2}+|B|^{2}\right)\left(\left|A_{x}\right|+|\mu A|\right)+\left|\mu A_{x x}\right|+\left|A_{x x x}\right|+|A|\left(|A|^{2}+|B|^{2}\right)^{2}\right]
$$

HORT2 $=\mathrm{O}\left[\left(\mu^{2}+|A|^{2}+|B|^{2}\right)\left(\left|B_{x}\right|+|\mu B|\right)+\left|\mu B_{x x}\right|+\left|B_{x x x}\right|+|B|\left\{|A|^{2}+|B|^{2}\right)^{2}\right]$.

The coefficients $b_{0}, b_{1}, c, d_{0}$ and $d_{1}$ and the vectors $U_{0}, \ldots, U_{5}$ are as obtained above (i.e. are given by $(2.6)-(2.13)$ ), while the remaining vectors in $(2.18)$ are given by

$\left(F_{1}-4 k_{0}^{2} D-2 \mathrm{i} \omega I\right) U_{6}=-\mathcal{B}\left(U_{0}, U_{0}\right)$

$F_{1} U_{7}=-2 \mathcal{B}\left(\bar{U}_{0}, U_{0}\right)$

$\left(F_{1}-2 \mathrm{i} \omega I\right) W_{1}=-2 \mathcal{B}\left(U_{0}, U_{0}\right)$

$\left(F_{1}-4 k_{0}^{2} D\right) W_{2}=-2 \mathcal{B}\left(\bar{U}_{0}, U_{0}\right)$

$\left(F_{1}-k_{0}^{2} D-\mathrm{i} \omega I\right) U_{8}=e_{1} U_{0}-2 \mathcal{B}\left(U_{7}, U_{0}\right)-2 \mathcal{B}\left(U_{6}, \bar{U}_{0}\right)-3 \mathcal{C}\left(U_{0}, U_{0}, \bar{U}_{0}\right)$

$\left(F_{1}-k_{i}^{2} D-\mathrm{i} \omega I\right) U_{9}=e_{2} U_{0}-2 \mathcal{B}\left(U_{7}+W_{2}, U_{0}\right)-2 \mathcal{B}\left(W_{1}, \bar{U}_{0}\right)-6 \mathcal{C}\left(U_{0}, U_{0}, \bar{U}_{0}\right)$.

Now. $U_{6}, U_{7} . W_{1}$ and $W_{2}$ are uniquely determined by $(2.21)-(2.24)$ because the $N \times N$ matrices in the left-hand sides of these equations are nonsingular (otherwise some of the points $(\Omega, k)=\left(2 \mathrm{i} \omega, 2 k_{0}\right),(0,0),\left(0,2 k_{0}\right)$ or $(2 \mathrm{i} \omega, 0)$ would satisfy the dispersion relation of the linearized eigenvalue problem for $\mu=0$, and this cannot happen, according to the assumption made right after (2.5)). Equations (2.25) and (2.26) instead possess a solution if and only if

$$
\begin{aligned}
& e_{1}=\bar{U}_{0}^{* T}\left[2 \mathcal{B}\left(U_{7}, U_{0}\right)+2 \mathcal{B}\left(U_{6}, \bar{U}_{0}\right)+3 \mathcal{C}\left(U_{0}, U_{0}, \bar{U}_{0}\right)\right] \\
& e_{2}=\bar{U}_{0}^{* T}\left[2 \mathcal{B}\left(U_{7}+W_{2}, U_{0}\right)+2 \mathcal{B}\left(W_{1}, \bar{U}_{0}\right)+6 \mathcal{C}\left(U_{0}, U_{0}, \bar{U}_{0}\right)\right]
\end{aligned}
$$

where $U_{0}^{*}$ is given by (2.14). These conditions determine the complex coefficients $e_{1}$ and $e_{2}$ in the amplitude equations (2.16). (2.17).

\subsection{The solution of (2.1), (2.2) in the boundary layers $|x \pm L / 2| \sim 1$}

Since the analysis of both boundary layers is completely similar, we only consider that one near $x=-L / 2$, where

$$
0<\xi=x+L / 2 \sim 1 .
$$

The steady state in this boundary layer is

$$
u=u^{0}(\xi)+\mu u^{1}(\xi)+\cdots
$$

where $u^{0}$ and $u^{1}$ are given by (see (2.1), (2.2))

$$
\begin{aligned}
& D u_{\xi \xi}^{0}+f\left(u^{0}, 0\right)=D u_{\xi \xi}^{1}+f_{u}\left(u^{0}, 0\right) u_{1}+f_{\mu}\left(u^{0}, 0\right)=0 \quad \text { in } 0<\xi<\infty \\
& C u^{0}-E u_{\xi}^{0}-W=C u^{1}-E u_{\xi}^{1}=0 \quad \text { at } \xi=0 \\
& \left|u^{0}\right|+\left|u^{1}\right| \rightarrow 0 \quad \text { as } \xi \rightarrow \infty .
\end{aligned}
$$


We assume that (2.31)-(2.33) uniquely determines $u^{0}$ (otherwise, (2.1), (2.2) either has no steady state that vanishes in the bulk, or it has more than one such steady state) and that the spectnum of the linearized eigenvalue problem

$$
\begin{aligned}
& D U_{\xi \xi}+f_{u}\left(u^{0}, 0\right) U=\Omega U \quad \text { in } 0<\xi<\infty \\
& C U-E U_{\xi}=0 \quad \text { at } \xi=0 \\
& U \rightarrow 0 \quad \text { as } \xi \rightarrow \infty
\end{aligned}
$$

has a strictly negative real part except for the spectral values $\Omega= \pm \mathrm{i} \omega$. that are associated with the oscillatory behaviour in the bulk (otherwise either the steady state (2.30) is exponentially unstable in the time scale $t \sim 1$ and the evolution of $u$ in the boundary layer is fully nonlinear, or the weakly nonlinear description in the boundary layer is more complex than that below). Then (2.31)-(2.33) uniquely determines $u^{1}$. Also, according to the assumption made at the beginning of section 2.1, the $N \times N$ matrix $f_{u}(0,0)+\lambda^{2} D$ is nonsingular whenever the complex constant $\lambda$ is purely imaginary: then the convergence (2.33) is exponential (see [55]).

The weakly nonlinear evolution of the solution of (2.1). (2.2) in the boundary layer is given by

$$
\begin{aligned}
u=u^{0}+\mu u^{1} & +\left[a U^{0}+a \mu U^{1}+a|a|^{2} U^{4}+a_{t} U^{5}+\text { HORT }\right] \mathrm{e}^{\mathrm{i} \omega t} \\
& +a^{2} U^{2} \mathrm{e}^{2 \mathrm{i} \omega t}+\text { c.c. }+|a|^{2} U^{3}+\text { HONRT }
\end{aligned}
$$

where HONRT (higher order non-resonant terms) stands for terms whose fast oscillatory part is of the type $\exp (\mathrm{im} \omega t)$ for some integer $m$ such that $m \neq \pm \mathrm{I}$. that are of higher order than those displayed, HORT (higher order resonant terms) stands for

$$
\text { HORT }=\mathrm{O}\left[\left(|\mu|+|a|^{2}\right)\left(\left|a_{t}\right|+|\mu a|+|a|^{3}\right)+\left|a_{t}\right|\right]
$$

and the complex amplitude $a$ is small and depends weakly on time. The functions $U^{0}, \ldots, U^{5}$ are given by

$D U_{\xi \xi}^{0}-\mathrm{i} \omega U^{0}+f_{u}\left(u^{0}, 0\right) U^{0}=0$

$D U_{\xi \xi}^{1}-\mathrm{i} \omega U^{1}+f_{u}\left(u^{0}, 0\right) U^{1}=-f_{u \mu}\left(u^{0}, 0\right) U^{0}-2 \mathcal{B}_{1}\left(u^{1}, U^{0}\right)$

$D U_{\xi \xi}^{2}-2 \mathbf{i} \omega U^{2}+f_{u}\left(u^{0}, 0\right) U^{2}=-\mathcal{B}_{1}\left(U^{0}, U^{0}\right)$

$D U_{\xi \xi}^{3}+f_{u}\left(u^{0}, 0\right) U^{3}=-2 \mathcal{B}_{1}\left(U^{0}, \bar{U}^{0}\right)$

$D U_{\xi \xi}^{4}-\mathrm{i} \omega U^{4}+f_{u}\left(u^{0}, 0\right) U^{4}=-2 \mathcal{B}_{1}\left(U^{3}, U^{0}\right)-2 \mathcal{B}_{1}\left(U^{2}, \bar{U}^{0}\right)-3 \mathcal{C}_{1}\left(U^{0}, U^{0}, \bar{U}^{0}\right)$

$D U_{\xi \xi}^{5}-i \omega U^{5}+f_{u}\left(u^{0}, 0\right) U^{5}=U^{0} \quad$ in $0<\xi<\infty$

$C U^{j}-E U_{\xi}^{j}=0 \quad$ at $\xi=0$

$\left|U^{j}\right|$ diverges at most algebraically as $\xi \rightarrow \infty$

for $j=1, \ldots, 5$, where the multilinear (symmetric) operators $\mathcal{B}_{1}$ and $\mathcal{C}_{1}$ are

$$
\mathcal{B}_{1} \equiv f_{u M}\left(u^{0}, 0\right) / 2 \quad \mathcal{C}_{1}=f_{u a u}\left(u^{0}, 0\right) / 6 .
$$

Also, since the convergence (2.33) is exponential, we have

$$
\left|u^{0}\right|+\left|u^{1}\right| \rightarrow 0 \quad\left|f_{u}\left(u^{0}, 0\right)-F_{1}\right|+\left|f_{\mu u}\left(u^{0}, 0\right)-F_{2}\right| \rightarrow 0
$$$$
\left|\mathcal{B}_{1}-\mathcal{B}\right| \rightarrow 0 \text { and }\left|\mathcal{C}_{1}-\mathcal{C}\right| \rightarrow 0 \quad \text { exponentially as } \xi \rightarrow \infty \text {. }
$$

where the matrices $F_{1}$ and $F_{2}$ and the multilinear operators $B$ and $\mathcal{C}$ were defined in (2.4). 
Let us first consider the linear equation (2.36). Its general solution is given by

$$
U^{0}=\sum_{k=1}^{N}\left[\alpha_{k}^{+} V_{+}^{k}(\xi)+\alpha_{k}^{-} V_{-}^{k}(\xi)\right]
$$

where, for $k=1, \ldots, N, \alpha_{k}^{ \pm}$are arbitrary constants. Also, since $u^{0} \rightarrow 0$ exponentially as $\xi \rightarrow \infty$, general results in, [55] imply that the linearly independent solutions $V_{ \pm}^{1} \ldots \ldots V_{ \pm}^{N}$ may be selected to behave asymptotically as $2 \mathrm{~N}$ prescribed solutions of the asymptotic problem $D U_{\xi \xi}-\mathbf{i} \omega U+f_{u}(0,0) U=0$. In particular. they may be selected such that

$$
\left|V_{ \pm}^{k}(\xi)-W_{ \pm}^{k}(\xi) \mathrm{e}^{ \pm \lambda_{k} \xi}\right|=\mathrm{o}\left(\left|W_{ \pm}^{k}(\xi) \mathrm{e}^{ \pm \lambda_{k} \xi}\right|\right) \quad \text { as } \xi \rightarrow \infty
$$

where $\left|W_{ \pm}^{k}\right|$ diverges at most algebraically as $\xi \rightarrow \infty$ and $\pm \lambda_{k}$ are the solutions of the characteristic equation

$$
\operatorname{det}\left[\lambda^{2} D-\mathbf{i} \omega I+f_{u}(0,0)\right]=0
$$

with $\operatorname{Re}\left(\lambda_{k}\right) \geqslant 0$ for $k=1, \ldots, N$. But. according to the assumption at the beginning of (2.1). two of those solutions are $\pm \lambda_{1}= \pm \mathrm{i} k_{0}$. while the remaining ones have a non-zero real part. Then, the solution (2.45) satisfies (2.43) only if $\alpha_{k}^{+}=0$ for $k=2, \ldots, N$. Thus (2.45) may be rewritten as

$$
U^{0}=\alpha_{1}^{+} V_{+}^{1}(\xi)+\alpha_{1}^{-} V_{-}^{1}(\xi)+\sum_{k=2}^{N} \alpha_{k}^{-} V_{-}^{k}(\xi)
$$

and

$\left|V_{+}^{1}-U_{\omega} \mathrm{e}^{\mathrm{i} k_{0} \xi}\right|+\left|V_{-}^{1}-U_{\omega} \mathrm{e}^{-1 k_{0} \xi}\right|+\sum_{k=2}^{N}\left|V_{-}^{k}(\xi)\right| \rightarrow 0$ exponentially, as $\xi \rightarrow \infty$

where the vector $U_{0}$ may be chosen to satisfy the last condition in (2.14).

Finally, we must impose the boundary conditions (2.42). We assume that

$$
\operatorname{dim} \operatorname{span}\left\{W^{0}, W^{2}, \ldots, W^{N}\right\}=\operatorname{dim} \operatorname{span}\left\{W^{1}, W^{2}, \ldots, W^{N}\right\}=N
$$

where $W^{0}=-E\left(\mathrm{~d} V_{+}^{1}(0) / \mathrm{d} \xi\right)+C V_{+}^{1}(0)$ and $W^{k}=-E\left(\mathrm{~d} V_{-}^{k}(0) / \mathrm{d} \xi\right)+C V_{-}^{k}(0)$ for $k=1, \ldots, N$; then by imposing $(2.42)$ to $(2.46)$ we obtain the following relations:

$$
\alpha_{k}^{-} / \alpha_{1}^{+}=r_{k} \quad \text { for } k=1, \ldots, N
$$

where $r_{1} \ldots r_{N}$ are given complex constants, and the solution (2.46) may be rewritten as

$$
U^{0}=V_{+}^{1}(\xi)+r_{1} V_{-}^{1}(\xi)+\sum_{k=2}^{N} r_{k} V_{-}^{k}(\xi)
$$

up to a constant factor. Now, according to (2.47),

$$
U^{0}(\xi)=U_{0}\left(\mathrm{e}^{i k_{0} \xi}+r_{1} \mathrm{e}^{-i k_{0} \xi}\right)+\text { EST } \quad \text { as } \xi \rightarrow \infty
$$

where EST stands for exponentially small terms.

The remaining linear problems (2.37)-(2.43) are seen to have a unique solution (modulo a solution of (2.36) in the case of the singular problems $(2.37),(2.40)-(2.43))$; for the sake of brevity we do not give here the (straightforward but somewhat tedious) proof of this assertion. 
For matching purposes we only need the asymptotic behaviour of $U^{1} \ldots, U^{5}$ as $\xi \rightarrow \infty$. When taking into account (2.44), (2.49) and using the same ideas as above, such behaviour is found to be as given by

$$
\begin{aligned}
& U^{1}= {\left[b_{0}^{-1} d_{0}\left(-\xi U_{0}+\mathrm{i} U_{1}\right)+U_{3}\right] \mathrm{e}^{\mathrm{i} k_{0} \xi}+r_{1}\left[b_{0}^{-1} d_{0}\left(\xi U_{0}+\mathrm{i} U_{1}\right)+U_{3}\right] \mathrm{e}^{-i k_{0} \xi}+\text { EST } } \\
& U^{2}= U_{6}\left(\mathrm{e}^{2 \mathrm{i}_{0} \xi}+r_{1}^{2} \mathrm{e}^{-2 k_{0} \xi}\right)+r_{1} W_{1}+\text { EST } \\
& U^{3}=\bar{r}_{1} W_{2} \mathrm{e}^{21 k_{0} \xi}+\text { c.c. }+\left(1+\left|r_{1}\right|^{2}\right) U_{7}+\text { EST } \\
& U^{4}=\left[b_{0}^{-1}\left(e_{1}+\left|r_{1}\right|^{2} e_{2}\right)\left(-\xi U_{0}+\mathrm{i} U_{1}\right)+U_{8}+\left|r_{1}\right|^{2} U_{9}\right] \mathrm{e}^{\mathrm{i} k_{0} \xi} \\
& \quad \quad+r_{1}\left[b_{0}^{-1}\left(e_{1}\left|r_{1}\right|^{2}+e_{2}\right)\left(\xi U_{0}+\mathrm{i} U_{1}\right)+\left|r_{1}\right|^{2} U_{8}+U_{9}\right] \mathrm{e}^{-\mathrm{i} k_{0} \xi}+\text { EST } \\
& U^{5}=b_{0}^{-1}\left(\xi U_{0}-\mathrm{i} U_{1}\right) \mathrm{e}^{\mathrm{i} k_{0} \xi}-r_{1} b_{0}^{-1}\left(\xi U_{0}+\mathrm{i} U_{1}\right) \mathrm{e}^{-1 k_{0} \xi}+\text { EST }
\end{aligned}
$$

where the vectors $U_{j}$ (for $\left.j=0, \ldots, 9\right), W_{1}$ and $W_{2}$ are solutions of $(2.6)-(2.11),(2.21)-$ (2.26) and the constants $b_{0}, c, d_{0}, e_{1}$ and $e_{2}$ are given by (2.12), (2.13) and (2.27), (2.28).

\subsection{Matching between the solutions in the bulk and in the boundary layers}

The boundary conditions to be applied to (2.16), (2.17) are obtained here from matching conditions between the solutions in the bulk and in the boundary layers. To this end, we first introduce the spatial variable $(2.29)$ and consider the limit

$$
1 \ll \xi \ll \lambda
$$

where $\lambda \gg I$ is the shorter space scale in the bulk. i.e. $\lambda$ is such that

$$
\lambda\left|A_{x}\right|=O(|A|) \text { and } \lambda\left|B_{x}\right|=O(|B|) \quad \text { in }-L / 2<x<L / 2 \text {. }
$$

In this limit, the solution in the bulk (see (2.18)) is given by

$$
\begin{aligned}
& u=\left[\left(A_{0}+A_{0 x} \xi\right) U_{0}-\mathbf{i} A_{0 x} U_{1}-A_{0 x x} U_{2}+\mu A_{j} U_{3}+A_{0}\left(\left|A_{j}\right|^{2} U_{8}\right.\right. \\
& \left.+\left|B_{0}\right|^{2} U_{9}\right)+ \text { HORTI] } \mathrm{e}^{\mathrm{i} \omega t+1 k_{0} \xi-1 k_{0} L / 2} \\
& +\left[\left(B_{0}+B_{0 x} \xi\right) U_{0}+\mathrm{i} B_{0 x} U_{1}-B_{0 x x} U_{2}+\mu B_{0} U_{3}+B_{0}\left(\left|B_{0}\right|^{2} U_{8}\right.\right. \\
& \left.\left.+\left|A_{0}\right|^{2} U_{9}\right)+ \text { HORT2 }\right] \mathrm{e}^{\mathrm{i} \omega t-i k_{0} \xi+i_{0} L / 2}+\text { c.c. }+ \text { NRT }
\end{aligned}
$$

and the solution in the boundary layer is (see (2.34), (2.49)-(2.54))

$$
\begin{aligned}
u=\left[a U_{0}+b_{0}^{-1}\left(a_{t}-d_{0} \mu a-\left(e_{1}+\left|r_{1}\right|^{2} e_{2}\right) a|a|^{2}\right)\left(\xi U_{0}-\mathrm{i} U_{1}\right)+a \mu U_{3}\right. \\
+a|a|^{2}\left(U_{8}+\left|r_{1}\right|^{2} U_{9}\right)+\text { HORT } \mathrm{e}^{\mathrm{i} \omega t+\mathrm{i} k_{0} \xi} \\
+r_{1}\left[a U_{0}+b_{0}^{-1}\left(d_{0} \mu a+\left(e_{1}\left|r_{1}\right|^{2}+e_{2}\right) a|a|^{2}-a_{t}\right)\left(\xi U_{0}+\mathrm{i} U_{1}\right)+a \mu U_{3}\right. \\
+a|a|^{2}\left(\left|r_{1}\right|^{2} U_{8}+U_{9}\right)+\text { HORT } \mathrm{e}^{\mathrm{k}\left(u t-\mathrm{i} k_{0} \xi\right.}+\text { c.c. }+\mathrm{NRT}
\end{aligned}
$$

where HORT1, HORT2 and HORT are as defined in (2.19), (2.20) and (2.35), NRT stands for non-resonant terms (i.e. terms whose fast oscillatory part is of the type $\exp \left(\mathrm{i} m \omega t+\mathrm{i} n k_{0} \xi\right)$, with $\left.\left|m^{2}-1\right|+\left|n^{2}-1\right| \neq 0\right)$, and

$$
A_{0}=A(-L / 2, t) \quad B_{0}=B(-L / 2, t) \quad A_{0 x}=A_{x}(-L / 2, t), \ldots
$$

Now. by identifying (2.57) and (2.58) we get. at $x=-L / 2$

$$
\begin{array}{ll}
A \mathrm{e}^{-\mathrm{i} k_{0} L / 2}=a+\text { HOT } & A_{x} \mathrm{e}^{-\mathrm{i} k_{0} L / 2}=b_{0}^{-1}\left[a_{t}-d_{0} \mu a-\left(e_{1}+\left|r_{1}\right|^{2} e_{2}\right) a|a|^{2}\right]+\text { HOT } \\
B \mathrm{e}^{\mathrm{i} k_{0} L / 2}=r_{1} a+\text { HOT } & B_{x} \mathrm{e}^{\mathrm{i} k_{0} L / 2}=b_{0}^{-1} r_{1}\left[d_{0} \mu a+\left(e_{1}\left|r_{1}\right|^{2}+e_{2}\right) a|a|^{2}-a_{t}\right]+\text { HOT }
\end{array}
$$


where

$$
\begin{aligned}
\text { HOT }= & \text { O[ }\left(|\mu|+|A|^{2}+|B|^{2}\right)\left(\left|A_{x}\right|+\left|B_{x}\right|+|\mu A|+|\mu B|\right) \\
& \left.+(|A|+|B|)\left(|\mu|+|A|^{2}+|B|^{2}\right)^{2}\right]+O\left[\left|A_{x x x}\right|+\left|B_{x x x}\right|+\left|A_{t t}\right|+\left|B_{t t}\right|\right] .
\end{aligned}
$$

Then

$B=r A+$ HOT $\quad b_{0}\left(B_{x}+r A_{x}\right)=r\left(|r|^{2}-1\right)\left(e_{1}-e_{2}\right) A|A|^{2}+$ HOT at $x=-L / 2$

where

$$
r=r_{1} \mathrm{e}^{-\imath k_{0} L}
$$

and the derivation of the boundary conditions (1.4), (1.5) at $x=-L / 2$ is complete. The boundary conditions at $x=L / 2$ are obtained, in a similar way, to be

$A=r B+$ HOT $\quad b_{0}\left(A_{x}+r B_{x}\right)=r\left(|r|^{2}-1\right)\left(e_{2}-e_{1}\right) B|B|^{2}+$ HOT $\quad$ at $x=L / 2$

where HOT and $r$ are given again by (2.60) and (2.62).

\section{The two asymptotic regimes for the amplitude equations}

We now consider the amplitude equations (2.16). (2.17) with the boundary conditions (2.61) and $(2.63)$ where as assumed earlier, the real parts of $c$ and $d_{0}$ are positive. In addition, we assume that the real part of the coefficient $e_{1}$, is negative. This is a supercriticality assumption for if the real part of $e_{1}$ is positive (resp., vanish). then we conjecture (although we were unable to prove it) that the problem $(2.16),(2.17),(2.61),(2.63)$ possesses solutions that blow up in finite time (resp., as $t \rightarrow \infty$ ); a further supercriticality assumption (namely, the real part of $e_{1}+e_{2}$ is negative) will be imposed below, in due course.

After replacing $A$ by $A \exp \left[\left(\mu\left(d_{0}-\bar{d}_{0}\right)+\mu^{2}\left(d_{1}-\bar{d}_{1}\right)\right) t / 2\right]$ and $B$ by $B \exp \left[\left(\mu\left(d_{0}-\right.\right.\right.$ $\left.\bar{d}_{0}\right)+\mu^{2}\left(d_{1}-\bar{d}_{1}\right) t t / 2$ ], respectively, and re-scaling $A, B, x, \mu$ and $L,(2.16),(2.17),(2.61)$ and (2.63) may be rewritten as (if $b_{0}>0$ ).

$$
\begin{aligned}
& A_{i}=\left(\mathrm{I}+\mathrm{i} \alpha_{2}\right) A_{x x}+\left[\mathrm{I}+\mu\left(\alpha_{6}+\mathrm{i} \alpha_{7}\right)\right] A_{x}+A\left[\mu+\alpha_{8} \mu^{2}-\left(1+\mathrm{i} \alpha_{3}\right)|A|^{2}\right. \\
& \left.-\left(\alpha_{1}+\mathrm{i} \alpha_{4}\right)|B|^{3}\right]+ \text { HORT1 } \quad \text { in }-L / 2<x<L / 2 \\
& B_{t}=\left(1+\mathrm{i} \alpha_{2}\right) B_{x x}-\left[1+\mu\left(\alpha_{6}+\mathrm{i} \alpha_{7}\right)\right] B_{x}+B\left[\mu+\alpha_{8} \mu^{2}-\left(1+\mathrm{i} \alpha_{3}\right)|B|^{2}\right. \\
& \left.-\left(\alpha_{1}+\mathrm{i} \alpha_{4}\right)|A|^{2}\right]+ \text { HORT } 2 \quad \text { in }-L / 2<x<L / 2 \\
& B=\rho \mathrm{e}^{1 \alpha_{s}} A+\text { HOT } \\
& B_{x}+\rho \mathrm{e}^{\mathrm{i} \alpha_{s}} A_{x}=\rho \mathrm{e}^{\mathrm{i} \alpha_{s}}\left(1-\rho^{2}\right)\left[1-\alpha_{1}+\mathrm{i}\left(\alpha_{3}-\alpha_{4}\right)\right] A|A|^{2}+\text { HOT } \\
& \text { at } x=-L / 2 \\
& A=\rho \mathrm{e}^{\mathrm{i} \alpha} B+\text { HOT } \\
& A_{x}+\rho \mathrm{e}^{\mathrm{i} \alpha_{\alpha}} B_{x}=\rho \mathrm{e}^{1 \alpha_{s}}\left(\mathrm{I}-\rho^{2}\right)\left[\alpha_{1}-\mathrm{I}+\mathrm{i}\left(\alpha_{4}-\alpha_{3}\right)\right] B|B|^{2}+\text { HOT } \\
& \text { at } x=L / 2
\end{aligned}
$$

where $\alpha_{1}, \ldots, \alpha_{8}$ are real,

$$
\rho=|r|>0
$$

and HORT1, HORT2 and HOT are as given in (2.19), (2.20) and (2.60). If the group velocity (at $\mu=0$ ) $b_{0}$ is negative. then (3.1). (3.4) are still obtained after replacing $A$ by $B$, $B$ by $A$ and $\rho$ by $\rho^{-1}$ everywhere; therefore. $\rho$ mav be larger than $I$ in (3.3), (3.4), even if 


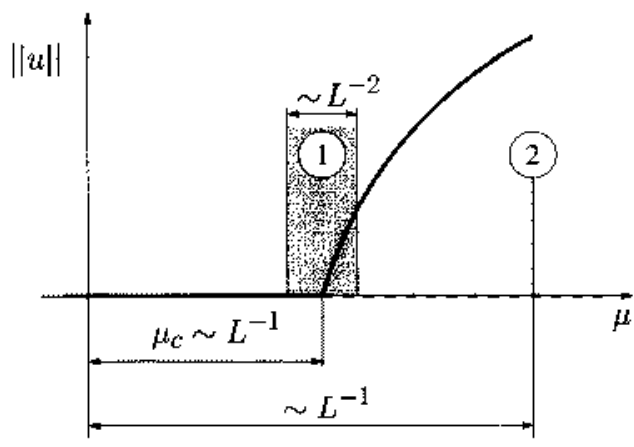

Figure 3. The two distinguished linits (3.7) and (3.8).

we are analysing the (frequent) case of absorbing boundaries (i.e. the reflection coefficient at the walls, $r$, such that $|r|<1$ ), and the group velocity is negative (then $\rho=|r|^{-1}>1$ ).

Now the trivial solution $(A=B=0)$ of $(3.1)-(3.4)$ is linearly stable if and only if

$$
\mu<\mu_{c} \equiv-L^{-1} \log \rho+\mathrm{O}\left(L^{-2}\right)
$$

as is readily seen. Notice that the fact that the spatial domain is finite and the wavetrains are reflected at the end-walls yields a shift in the instability limit of the basic state. that may be explained as follows. If the group velocitv $b_{0}$ is positive and $\rho>\mathbf{I}$ (resp., $\rho<\mathbf{1}$ ) then the wavetrains are amplified (resp.. absorbed) at the end-walls and this has a destabilizing (resp.. stabilizing) effect that must be compensated in the bulk: if, instead. the group velocity is negative then amplifying and absorbing boundary conditions have stabilizing and destabilizing effects respectively. According to (3.6) we are led to consider the distinguished sub-limits (see figure 3)

$$
\begin{aligned}
& |A|^{2} \sim|B|^{2} \sim\left|\mu-\mu_{c}\right| \sim L^{-2} \\
& |A|^{2} \sim|B|^{2} \sim\left|\mu-\mu_{c}\right| \sim L^{-1}
\end{aligned}
$$

of the limit $|\mu| \ll 1, L \gg 1$, with $A$ and $B$ satisfying (1.3). Now if $L \gg 1$ is kept fixed and the bifurcation parameter $\mu$ is varied then the first limit is concerned with a quite narrow range near the instability limit (figure 3 ), while the second limit accounts for a wider range and thus has a wider scope. If instead $\mu-\mu_{c}$ is kept fixed (and such that $\left|\mu-\mu_{c}\right| \ll 1$ ) then the first limit is concerned with smaller domains $\left(L \sim\left|\mu-\mu_{c}\right|^{-1 / 2}\right)$ and the second limit to larger domains $\left(L \sim\left|\mu-\mu_{c}\right|^{-1} \sim|\mu|^{-1}\right)$, and they may be called small and large domain limits (or regimes), respectively. These two limits are considered below. in sections 4 and 5 .

\section{The averaged Ginzburg-Landau equation in the small domain regime (3.7)}

In the limit (3.7) we shall obtain a non-local Ginzburg-Landau equation (NLGLE) giving the slow evolution of the complex amplitudes in first approximation. After obtaining the NLGLE by means of a two-timing scales method (in section 4.1), we shall give some preliminary properties of the model (in section 4.2), along with the results of some numerical calculations.

\subsection{Asymptotic derivation of the model equation}

In order to remove the reflection coefficient from the first boundary conditions in (3.3)(3.4). and to introduce the appropriate scaling in the limit (3.7), we define the new complex 
amplitudes, $Y$ and $Z$, the re-scaled space and time variables, $\xi$ and $T$, the new bifurcation parameter $\lambda$ and the small parameter $\varepsilon$, as

$$
\begin{aligned}
& A=\varepsilon \rho^{\xi+1 / 2} Y \exp \left[\mathrm{i} \alpha_{5}(\xi+T+1 / 2)+\mathrm{i} \varepsilon\left(\left(\alpha_{2}-\alpha_{7}\right)(\log \rho)^{2}+\alpha_{5}\left(2-\alpha_{6}\right) \log \rho-\alpha_{2} \alpha_{5}^{2}\right) T\right] \\
& B=\varepsilon \rho^{-\xi+1 / 2} Z \exp \left[\mathrm{i} \alpha_{5}(-\xi+T+1 / 2)+\mathrm{i} \varepsilon\left(\left(\alpha_{2}-\alpha_{7}\right)(\log \rho)^{2}\right.\right. \\
& \left.\left.\quad \quad+\alpha_{5}\left(2-\alpha_{6}\right) \log \rho-\alpha_{2} \alpha_{5}^{2}\right) T\right] \\
& \begin{array}{l}
\xi=\varepsilon x \quad T=\varepsilon t \\
\mu
\end{array}=-\varepsilon \log \rho+\varepsilon^{2}\left[\lambda+\alpha_{5}^{2}+\alpha_{5}\left(2 \alpha_{2}-\alpha_{7}\right) \log \rho-\left(1+\alpha_{8}-\alpha_{6}\right)(\log \rho)^{2}\right] \\
& \varepsilon=1 / L \ll \mathbf{1}
\end{aligned}
$$

to write $(3.1)-(3.4)$ as

$$
\begin{aligned}
& Y_{T}=\varepsilon\left(1+\mathrm{i} \alpha_{2}\right) Y_{\xi \xi}+\left[1+\varepsilon\left(\alpha_{6}^{\prime}+\mathrm{i} \alpha_{5}^{\prime}\right)\right] Y_{\xi}+\varepsilon Y\left[\lambda-\left(1+\mathrm{i} \alpha_{3}\right) \rho^{1+2 \xi}|Y|^{2}\right. \\
& \left.\quad-\left(\alpha_{1}+\mathrm{i} \alpha_{4}\right) \rho^{1-2 \xi}|Z|^{2}\right]+\mathrm{O}\left(\varepsilon^{2}\right) \\
& Z_{T}=\varepsilon\left(1+\mathrm{i} \alpha_{2}\right) Z_{\xi \xi}-\left[1+\varepsilon\left(\alpha_{6}^{\prime}+\mathrm{i} \alpha_{5}^{\prime}\right)\right] Z_{\xi}+\varepsilon Z\left[\lambda-\left(1+\mathrm{i} \alpha_{3}\right) \rho^{1-\frac{2 \xi}{\xi}}|Z|^{2}\right. \\
& \left.\quad-\left(\alpha_{1}+\mathrm{i} \alpha_{4}\right) \rho^{1+2 \xi}|Y|^{2}\right]+\mathrm{O}\left(\varepsilon^{2}\right) \quad \text { in }-1 / 2<\xi<1 / 2 \\
& Y=Z+O\left(\varepsilon^{2}\right) \\
& Y_{\xi}+Z_{\xi}= \pm \varepsilon\left(\rho^{2}-1\right)\left[1-\alpha_{1}+\mathrm{i}\left(\alpha_{3}-\alpha_{4}\right)\right] Y|Y|^{2}+\mathrm{O}\left(\varepsilon^{2}\right) \quad \text { at } \xi= \pm 1 / 2
\end{aligned}
$$

where

$$
\alpha_{5}^{\prime}=\left(2 \alpha_{2}-\alpha_{7}\right) \log \rho+2 \alpha_{5} \quad \alpha_{6}^{\prime}=\left(2-\alpha_{6}\right) \log \rho-2 \alpha_{2} \alpha_{5} .
$$

Notice that the factors $\rho^{\xi+1 / 2}$ and $\rho^{-\xi+1 / 2}$ have been introduced in (4.1), (4.2) for the resulting problem to exhibit perfectly reflecting boundary conditions; this will allow us to apply a reflection principle and extend the spatial domain to $-\infty<\xi<\infty$ (thus the characteristics of the leading hyperbolic problem are straight lines and the application of solvability conditions will be much facilitated). The other factors in (4.1), (4.2) are introduced to eliminate the phase shift in the reflecting boundary conditions. and to obtain equations on the new variables that are as simple as posible.

Now, to the leading order, $Y$ and $Z$ satisfy linear, first-order, wave equations $\left(Y_{T}-Y_{\xi}=\right.$ $Z_{T}+Z_{\xi}=0$ in $\left.-1 / 2<\xi<1 / 2\right)$ with perfectly reflecting boundaries $(Y=Z$ at $\xi= \pm 1 / 2$ ). and thus exhibit a linear, undamped, hyperbolic behaviour. Diffusion. dispersion and nonlinearity come into play in a second, still slower, time scale

$$
\tau=\varepsilon T \quad\left(=\varepsilon^{2} t\right)
$$

and their effect is analysed by considering higher order terms

$$
Y=Y_{0}+\varepsilon Y_{1}+\cdots \quad Z=Z_{0}+\varepsilon Z_{1}+\cdots
$$

in (4.5)-(4.7). Then the following problems result:

$$
\begin{aligned}
Y_{0 T}-Y_{0 \xi}= & Z_{0 T}+Z_{0 \xi}=0 \\
Y_{1 T}-Y_{1 \xi}= & -Y_{0 \tau}+\left(1+\mathrm{i} \alpha_{2}\right) Y_{0 \xi \xi}+\left(\alpha_{6}^{\prime}+\mathrm{i} \alpha_{5}\right) Y_{0 \xi}+\lambda Y_{0} \\
& -Y_{0}\left[\left(1+\mathrm{i} \alpha_{3}\right) \rho^{1+2 \xi}\left|Y_{0}\right|^{2}+\left(\alpha_{1}+\mathrm{i} \alpha_{4}\right) \rho^{1-2 \xi}\left|Z_{j}\right|^{2}\right] \\
Z_{1 T}+Z_{1 \xi}= & -Z_{0 \tau}+\left(1+\mathrm{i} \alpha_{2}\right) Z_{0 \xi \xi}-\left(\alpha_{6}^{\prime}+\mathrm{i} \alpha_{\xi}^{\prime}\right) Z_{0 \xi}+\lambda Z_{0} \\
& -Z_{j}\left[\left(1+\mathrm{i} \alpha_{3}\right) \rho^{1-2 \xi}\left|Z_{j}\right|^{2}+\left(\alpha_{1}+\mathrm{i} \alpha_{4}\right) \rho^{1+2 \xi}\left|Y_{j}\right|^{2}\right] \quad \text { in }-1 / 2<\xi<1 / 2
\end{aligned}
$$

$Y_{0}-Z_{0}=Y_{0 \xi}+Z_{0 \xi}=Y_{1}-Z_{1}=0$

$Y_{1 \xi}+Z_{1 \xi}=\mp\left(\mathbf{l}-\rho^{2}\right)\left[1-\alpha_{1}+\mathrm{i}\left(\alpha_{3}-\alpha_{4}\right)\right] Y_{0}\left|Y_{i}\right|^{2} \quad$ at $\xi= \pm \mathbf{I} / 2$. 
Notice that the second and fourth pairs of boundary conditions in (4.14) need not be imposed because they readily follow from (4.11)-(4.13) and the remaining boundary conditions. Equations (4.11)-(4.13) are invariant under space reflection $\xi \rightarrow-\xi, Y_{0} \leftrightarrow Z_{0}$, $Y_{1} \leftrightarrow Z_{1}$. and the boundary conditions (4.14) are appropriate to apply a reflection principle at $\xi= \pm 1 / 2$ to extend (4.11)-(4.14) to the whole line. $-\infty<\xi<\infty$. with a spatial periodicity condition (as it is convenient to solve (4.11)-(4.14) in the faster time scale). In order to do that we introduce the new dependent variables $w_{0}$ and $w_{1}$. defined in $-\infty<\xi<\infty$ as

$$
\begin{array}{lc}
w_{j}(\xi, T, \tau)=Z_{j}(-1-\xi, T, \tau) & \text { if }-3 / 2 \leqslant \xi<-1 / 2 \\
w_{j}(\xi, T, \tau)=Y_{j}(\xi, T, \tau) \quad \text { if }-1 / 2 \leqslant \xi<1 / 2 \\
w_{j}(\xi+2, T, \tau)=w_{j}(\xi, T, \tau) & \text { if }-\infty<\xi<\infty
\end{array}
$$

for $j=0$ and 1 , to rewrite $(4.11)-(4.14)$ as

$$
\begin{aligned}
w_{0 T}-w_{0 \xi}= & 0 \\
w_{1 T}-w_{1 \xi}= & -w_{0 \tau}+\left(1+\mathrm{i} \alpha_{2}\right) w_{0 \xi \xi}+\left(\alpha_{6}^{\prime}+\mathrm{i} \alpha_{5}^{\prime}\right) w_{0 \xi}+\lambda w_{0}-w_{0}\left[\left(1+\mathrm{i} \alpha_{3}\right) \varphi(\xi)\left|w_{0}\right|^{2}\right. \\
& \left.+\left(\alpha_{1}+\mathrm{i} \alpha_{4}\right) \varphi(-\xi)\left|w_{0}(-1-\xi, T, \tau)\right|^{2}\right]
\end{aligned}
$$

where

$$
\begin{array}{lr}
\varphi(\xi)=\rho^{1+2 \xi} & \text { if }-1 / 2 \leqslant \xi \leqslant 1 / 2 \\
\varphi(\xi+1)=\varphi(\xi) & \text { if }-\infty<\xi<\infty
\end{array}
$$

Notice that if $0<\rho \neq 1$ then $\varphi$ exhibits jumps at $\xi-1 / 2=$ integer and thus $w_{1 \xi}$ is not smooth at these values of $\xi$. These jumps in $w_{1 \xi}$ were to be expected and they are precisely such that the boundary conditions (4.14) are satisfied, as is readily seen when taking into account the reflection principle (4.15). Now by integrating (4.17), (4.18) in the faster time scale we obtain

$$
\begin{aligned}
w_{0}=W(\eta, \tau) & \\
w_{1}=F(\eta, \tau)+ & (\zeta / 2)\left[-W_{\tau}+\left(1+\mathrm{i} \alpha_{2}\right) W_{\eta \eta}+\left(\alpha_{6}^{i}+\mathrm{i} \alpha_{5}^{i}\right) W_{\eta}+\lambda W\right] \\
& +\left(1+\mathrm{i} \alpha_{3}\right) W|W|^{2} \int_{0}^{(\eta-\zeta) / 2} \varphi(z) \mathrm{d} z \\
& +\left(\alpha_{1}+\mathrm{i} \alpha_{4}\right) W \int_{0}^{(\eta-\zeta) / 2} \varphi(-z)|W(\eta-2 z-1, \tau)|^{2} \mathrm{~d} z
\end{aligned}
$$

in terms of the characteristic variables

$$
\eta=T+\xi \quad \text { and } \quad \xi=T-\xi
$$

where the function $F$ remains undetermined at this stage in the perturbation process. Finally, by eliminating secular terms, i.e. by requiring $\omega_{1}$ to be bounded as $T \rightarrow \infty$ for each fixed value of $\eta$, we get

$$
\begin{aligned}
& W_{\tau}=\left(1+\mathrm{i} \alpha_{2}\right) W_{\eta \eta}+\left(\alpha_{6}^{\prime}+\mathrm{i} \alpha_{5}^{\prime}\right) W_{\eta}+\lambda W-\left[\left(\rho^{2}-\mathbf{1}\right) /(2 \log \rho)\right] W \\
& \times\left[\left(\mathbf{1}+\mathbf{i} \alpha_{3}\right)|W|^{2}+\left(\alpha_{1}+\mathbf{i} \alpha_{4}\right) \int_{-1 / 2}^{1 / 2} \phi(z)|W(\eta-2 z-1, \tau)|^{2} \mathrm{~d} z\right] \\
& W(\eta+2, \tau)=W(\eta, \tau) \quad \text { if }-\infty<\eta<\infty
\end{aligned}
$$

where

$$
\phi(z)=(2 \log \rho) \rho^{1-2 z} /\left(\rho^{2}-1\right) .
$$


To obtain (4.23) we have taken into account that, as $T \rightarrow \infty$ with $\eta$ fixed, $\zeta \rightarrow \infty$ and

$$
\begin{aligned}
& \int_{0}^{(\eta-\zeta) / 2} \varphi(z) \mathrm{d} z=-\left(\rho^{2}-1\right) \zeta /(4 \log \rho)+\mathrm{O}(1) \\
& \int_{0}^{(\eta-\zeta) / 2} \varphi(-z)|W(\eta-2 z-1, \tau)|^{2} \mathrm{~d} z=-(\zeta / 2) \int_{-1 / 2}^{1 / 2} \rho^{1-2 z}|W(\eta-2 z-1, \tau)|^{2} \mathrm{~d} z+\mathrm{O}(1)
\end{aligned}
$$

as readily seen when taking into account (4.16), (4.19) and (4.20).

Finally, by using the new variables $v$ and $y$, and the new bifurcation parameter $\beta$, defined as

$$
\begin{aligned}
& v=W\left[\left(\rho^{2}-1\right) /(2 \log \rho)\right]^{1 / 2} \exp \left[-\mathrm{i} \delta \eta+\mathrm{i} \delta\left(\delta \alpha_{2}-\alpha_{6}^{\prime}\right) \tau\right] \\
& y=\eta+\left(\alpha_{6}^{\prime}-2 \delta \alpha_{2}\right) \tau \quad \beta=\lambda-\delta^{2}-\delta \alpha_{5}^{\prime}
\end{aligned}
$$

where

$$
\left.\delta=-\pi \text { [integer part of }\left(\alpha_{5}^{\prime} / \pi\right)\right]
$$

equation (4.23) may be somewhat simplified to

$$
\begin{aligned}
& v_{\tau}=\left(1+\mathbf{i} \alpha_{2}\right) v_{y y}+\mathbf{i} \alpha_{5}^{\prime \prime} v_{y} \\
& +v\left[\beta-\left(1+\mathrm{i} \alpha_{3}\right)|v|^{2}-\left(\alpha_{1}+\mathbf{i} \alpha_{4}\right) \int_{-1 / 2}^{1 / 2} \phi(z)|v(y-2 z-1, \tau)|^{2} \mathrm{~d} z\right]
\end{aligned}
$$

$v(y+2, \tau)=v(y, \tau) \quad$ in $-\infty<y<\infty$

with

$$
\left.\left.\alpha_{5}^{\prime \prime}=2 \pi \text { [fractional part of }\left(\left(\alpha_{5}^{\prime}+\pi\right) / 2 \pi\right)\right)\right]-\pi .
$$

The averaged Ginzburg-Landau equation (4.27) depends on the bifurcation parameter $\beta$ and on the real parameters

$0<\rho<\infty \quad-\infty<\alpha_{j}<\infty$ for $j=1, \ldots, 4 \quad-\pi \leqslant \alpha_{5}^{\prime \prime}<\pi$.

Each solution of (4.27). (4.28) yields (a first approximation of) an evolution of the complex amplitudes $A$ and $B$. that may be calculated in terms of the time scales $T=\varepsilon t$ and $\tau=\varepsilon^{3} t$, when taking into account (4.1)-(4.4), (4.10), (4.15), (4.16), (4.20) and (4.26).

Notice that in the particular case of perfectly reflecting boundaries, $\rho=1$, a removable singularity appears in (4.23) and (4.25). If the analysis above is repeated for that particular case (not considered independently for the sake of brevity) then it is readily seen that everything stands after replacing $2(\log \rho) /\left(\rho^{2}-1\right)$ by 1 ; then the weight function $\phi$ appearing in the averaged term becomes identically equal to 1 .

The averaged term in (4.27) comes from the nonlinear cross-terms in (3.1). (3.2), that account for the effect of each wavetrain on the other (counterpropagating) one. It may be explained as follows. In the short time scale. $T=\varepsilon t \sim 1$. both counterpropagating wavetrains exhibit only linear propagation and reflection at the walls. The propagating velocity is large (of the order of $\varepsilon^{-1}$ ) in the slower time scale, $\tau=\varepsilon^{2} t \sim 1$. Thus, in the slower time scale each wavetrain 'sees' the other one travelling very fast in the opposite direction, and reflecting many times at the walls; only a weighted, averaged effect is felt in this time scale, the weight coming from the fact that the wavetrains are amplified (if $\rho<1$ ) or reduced (if $\rho>1$ ) as they travel, in order to counterbalance the instantaneous reduction (if $\rho<1$ ) or amplification (if $\rho>1$ ) at the walls.

A complete parametric description of the attractors (as $\tau \rightarrow \infty$ ) of (4.27). (4.28) is expected to be extremely complex due to both the presence of diffusion, dispersion, 
nonlinearity and mean-field effects, and the large number of parameters involved. Instead, some remarkable features will be outlined now. For a more complete analysis of (4.27), (4.28), see [42].

\subsection{Some properties of the averaged Ginzburg-Landau model}

A rigorous derivation of the averaged Ginzburg-Landau problem (4.27), (4.28) for the particular case of perfectly reflecting boundaries, $\rho=1$, was given in [39]. along with several global properties of the solutions. The ideas in [39] may be extended to the general case $\rho \neq 1$, to prove, in particular, that if initial data is prescribed. then (4.27), (4.28) define a unique solution, that is uniformly bounded for all $\tau \geqslant 0$ if $\alpha_{1}>-1$. If $\alpha_{1}<-1$ (resp.. $\alpha_{1}=-1$ ) then $(4.27),(4.28)$ possess solutions that blow up in finite time (resp.. as $\tau \rightarrow \infty$ ), as is readily seen when considering spatially uniform solutions, that are such that $V=|v|$ satisfy the following ODE:

$$
\mathrm{d} V / \mathrm{d} \tau=\left[\beta-\left(1+\alpha_{1}\right) V^{2}\right] V .
$$

Then, if $\alpha_{1}<-1$ (resp., $\alpha_{1}=-1$ ) local bifurcation at the onset of the oscillatory instability is sub-critical (resp., critical) and the analysis of the large-time dynamics of the underlying physical system requires that we consider the fully nonlinear model (resp.. requires that we consider higher order nonlinear terms in the amplitude equations). In what follows, we shall consider only the super-critical case, $\alpha_{1}>-1$.

The simplest solutions of $(4.27),(4,28)$ are such that $|v|$ is constant, and are given by

$$
v=R_{0} \exp \left(\mathrm{i} q_{0} \tau+\mathrm{i} p_{0} \pi y\right)
$$

where the real parameters $R_{0}>0$ and $q_{0}$ are given by

$$
\begin{aligned}
& R_{j}^{2}=\left[\beta-\left(p_{0} \pi\right)^{2}-\alpha_{5}^{\prime \prime}\left(p_{0} \pi\right)\right] /\left(1+\alpha_{1}\right) \\
& q_{0}=-\alpha_{2}\left(p_{0} \pi\right)^{2}-\left(\alpha_{3}+\alpha_{4}\right) R_{0}^{2}
\end{aligned}
$$

and $p_{0}$ is an arbitrary integer. Notice that (i) there are infinitely many branches of these solutions that bifurcate (through pitchfork bifurcations) from the basic steady state, $v=0$; (ii) these solutions are independent of the reflection coefficient $\rho$; and (iii) the associated solutions of the amplitude equations are fairly simple. such that $|A|=\varepsilon R_{0} \rho^{\xi+1 / 2}$ and $|B|=\varepsilon R_{\mathcal{U}} \rho^{-\xi+1 / 2}$ to a first approximation (see (4.1)-(4.4), (4.10). (4.15), (4.16). (4.20) and (4.26)). The (straightforward but tedious) linear stability analysis of these solutions provides some insight into the large-time dynamics of (4.27), (4.28) (see [42]). According to the consistency conditions (1.3), only a large but finite number of the infinitely many branches mentioned above correspond to approximate solutions of the underlying physical problem. These solutions are associated with the different possible values of the phase shifts of the complex amplitudes (of the counterpropagating wavetrains) between the end-walls, and (somehow) account for the discrete distribution of wavenumbers due to the fact that the spatial domain is large but finite.

Let us first consider the particular case of perfectly reflecting boundaries. $\rho=1$. If $1+\alpha_{2} \alpha_{3}<0$ and $\beta>0$ is not too small then all solutions (4.31) are unstable (they exhibit the modulational instability, as the simplest solutions of the standard Ginzburg-Landau equation do). Then the dynamics of (4.27), (4.28) is expected to be fairly complex, as shown in figure 4 , where representative results are given that were obtained by numerical integration of (4.27), (4.28) in the particular case $\rho=1, \alpha_{1}=3, \alpha_{2}=-1, \alpha_{3}=5, \alpha_{4}=\alpha_{5}^{\prime \prime}=0$, for several values of the bifurcation parameter $\beta$. In order to appreciate the spatial structure of $|v|$ to some extent. we have plotted $\|v\|=\left[\int_{-1}^{1}|v(y, \tau)|^{2} \mathrm{dy} / 2\right]^{1 / 2} \cdot|v(0, \tau)|$ and $|v(1, \tau)|$ in 

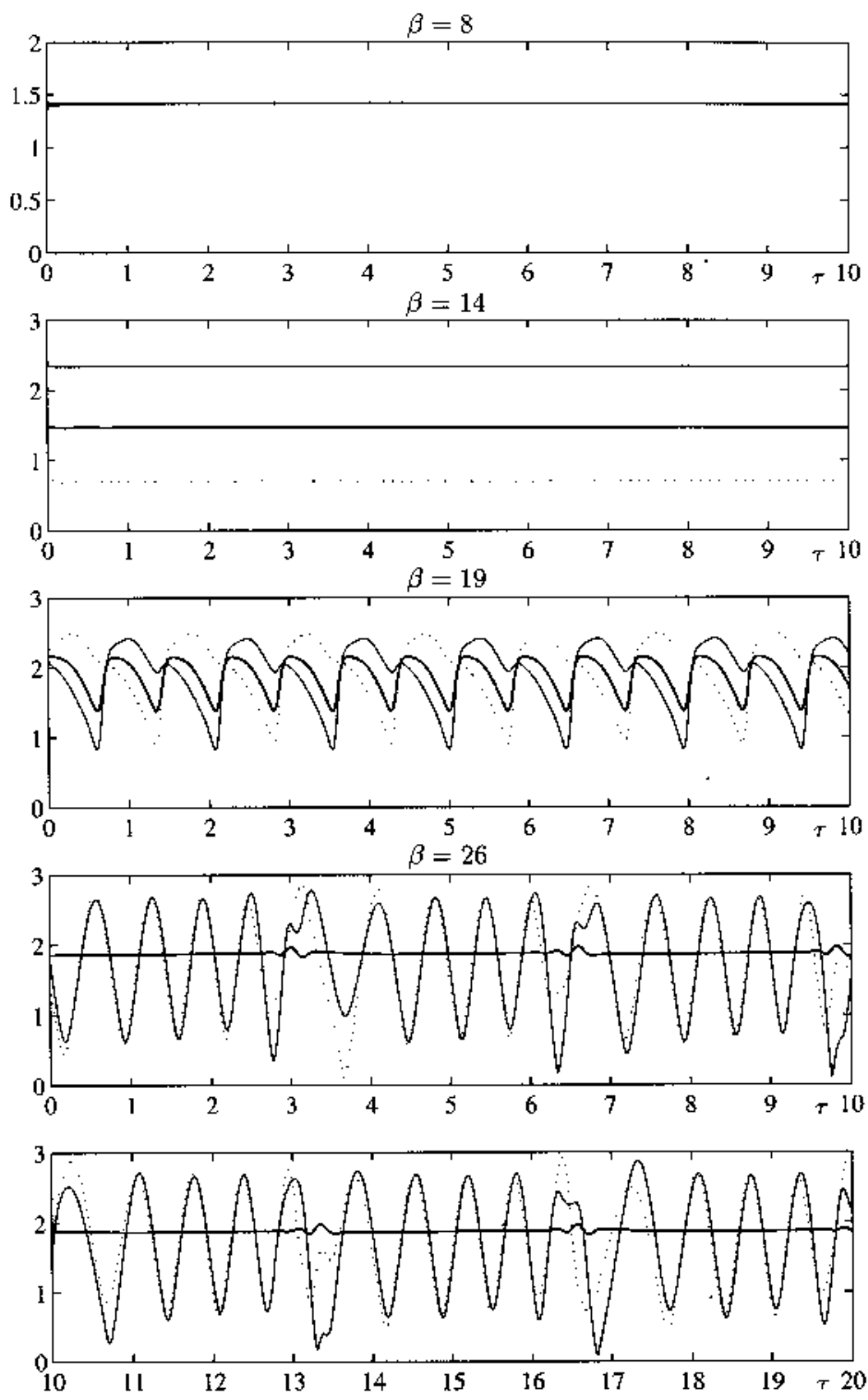

Figure 4. $\|v\|$ (thick full line), $|v(0, \tau)|$ (full curve) and $|v(1, \tau)|$ (dotted curve) versus $\tau$ as obtained by numerical integration of the NLGLE for $\rho=1, \alpha_{1}=3, \alpha_{2}=-1, \alpha_{3}=5$, $\alpha_{4}=\alpha_{5}^{\prime \prime}=0$ and the indicated values of $\beta$.

terms of $\tau$. For $\beta=8, v$ evolves to one of the solutions (4.31) as $\tau \rightarrow \infty$. For $\beta=14$, v evolves to a steady solution such that $|v|$ is not spatially uniform; when taking into account the changes of variables made in section 4.1 , it is seen that the associated solutions of the amplitude equations (3.1) and (3.2) is such that $A$ and $B$ alternatively (in the short time scale, $T=\varepsilon t \sim 1$ ) dominates in a part of the spatial domain $0<\xi<1$, and this corresponds to a 'blinking state', as first pointed out in [37]. For $\beta=19, v$ evolves to a limit cycle; the associated solution of the amplitude equations corresponding also to the blinking state 
whose structure now periodically evolves also in the slower time scale, $\tau=\varepsilon^{2} t \sim 1$. For $\beta=26$ the solution exhibits an intermittent behaviour as $\tau \rightarrow \infty$. After an interval of time when the profile behaves as a travelling wavetrain (the associated solutions of the amplitude equations corresponding to slowly modulated blinking states. as above), sudden excursions to more complex behaviour take place (at $\tau \simeq 3,6.5,9.5, \ldots$ ) in an aperiodic way. Such intermittent behaviour has also been obtained for the standard Ginzburg-Landau equation [56]; as in [56], for very large values of $\beta$, the solution of (4.27), (4.28) is expected to exhibit spatio-temporal intermittency, that will be considered in [42]. If $I+\alpha_{2} \alpha_{3}>0$. then the steady states (4.31) exhibit again instabilities for appropriate values of the remaining parameters, that are not considered here for the sake of brevity; if, in addition, $\beta$ is large, then the solutions of $(4.27),(4.28)$ are expected to exhibit a simple behaviour as suggested by the solutions in the second regime that will be considered in section 5 .

If $\rho \neq 1$, then the analysis of the linear stability of the solutions of $(4.31)$ is quite tedious. Here we only point out a remarkable feature concerning the limits $\rho \rightarrow 0$ and $\rho \rightarrow \infty$, that may be easily explained. If either $\rho \gg 1$ or $\rho \ll 1$, then the weight function $\phi$ appearing in the averaged term of (4.27) is close to a Dirac delta function, and (4.27) becomes the following standard Ginzburg-Landau equation:

$$
v_{\tau}=\left(\mathbf{I}+\mathrm{i} \alpha_{2}\right) v_{y y}+\mathrm{i} \alpha_{5}^{\prime \prime} v_{y}+v\left[\beta-\left(\mathbf{I}+\alpha_{1}+\mathbf{i}\left(\alpha_{3}+\alpha_{4}\right)\right)|v|^{2}\right] .
$$

For this approximation to be valid, the bifurcation parameter $\beta$ cannot be too large if $\rho$ remains fixed (for, if $\beta$ is large then $\left|v_{y}\right|$ is also large and the local approximation of the non-local averaged term fails). Then if $1+\alpha_{2}\left(\alpha_{3}+\alpha_{4}\right) /\left(1+\alpha_{1}\right)<0$ and $\beta>0$ is neither too large nor too small, then the solutions (4.31) exhibit the modulational instability; consequently, a complex dynamic behaviour is to be expected for intermediate values of $\beta$. Numerical integrations of (4.27), (4.28) show that this is in fact the case, as shown in figure 5, for the particular case $\rho=100, \alpha_{1}=3, \alpha_{2}=-1, \alpha_{3}=0, \alpha_{4}=6$ and $\alpha_{5}^{\prime \prime}=0$. As in figure 4, $\|v\|,|v(0, \tau)|$ and $|v(1, \tau)|$ are plotted in terms of $\tau$ for several values of the bifurcation parameter $\beta$.

\section{The real nonlinear wave system in the large domain regime (3.8)}

In the limit (3.8) we re-scale the amplitudes, the space and time variables and the bifurcation parameter as

$A=\varepsilon^{1 / 2} Y \quad B=\varepsilon^{1 / 2} Z \quad x=\xi / \varepsilon \quad t=T / \varepsilon \quad \mu=\varepsilon \lambda$

in terms of the small parameter

$$
\varepsilon=1 / L \ll 1
$$

to re-write $(3.1)-(3.4)$ as

$$
\begin{aligned}
& Y_{T}=\varepsilon\left(1+\mathrm{i} \alpha_{2}\right) Y_{\xi \xi}+Y_{\xi}+Y\left[\lambda-\left(1+\mathrm{i} \alpha_{3}\right)|Y|^{2}-\left(\alpha_{1}+\mathrm{i} \alpha_{4}\right)|Z|^{2}\right]+\text { HOT } \\
& Z_{T}=\varepsilon\left(1+\mathrm{i} \alpha_{2}\right) Z_{\xi \xi}-Z_{\xi}+Z\left[\lambda-\left(1+\mathrm{i} \alpha_{3}\right)|Z|^{2}-\left(\alpha_{1}+\mathrm{i} \alpha_{4}\right)|Y|^{2}\right]+\text { HOT } \\
& \quad \text { in }-1 / 2<\xi<1 / 2 \\
& Z=\rho \mathrm{e}^{\mathrm{i} \alpha_{s}} Y+\varepsilon \text { HOT at } \xi=-1 / 2 \quad Y=\rho \mathrm{e}^{\mathrm{i} \alpha_{5}} Z+\varepsilon \text { HOT } \quad \text { at } \xi=1 / 2 \\
& Z_{\xi}+\rho \mathrm{e}^{\mathrm{i} \alpha_{s}} Y_{\xi}=\rho \mathrm{e}^{\mathrm{i} \alpha_{s}}\left(1-\rho^{2}\right)\left[1-\alpha_{1}+\mathrm{i}\left(\alpha_{3}-\alpha_{4}\right)\right] Y|Y|^{2}+\text { HOT } \quad \text { at } \xi=-1 / 2 \\
& Y_{\xi}+\rho \mathrm{e}^{\mathrm{i} \alpha_{s}} Z_{\xi}=\rho \mathrm{e}^{i \alpha_{s}}\left(\rho^{2}-1\right)\left[1-\alpha_{1}+\mathrm{i}\left(\alpha_{3}-\alpha_{4}\right)\right] Z|Z|^{2}+\text { HOT } \quad \text { at } \xi=1 / 2
\end{aligned}
$$

where

$\mathrm{HOT}=\mathrm{O}\left[\varepsilon\left(\lambda+|Y|^{2}-|Z|^{2}\right)\left(\left|Y_{\xi}\right|+\left|Z_{\xi}\right|\right)+\varepsilon\left(\lambda+|Y|^{2}+|Z|^{2}\right)^{2}(|Y|+|Z|)\right]$. 

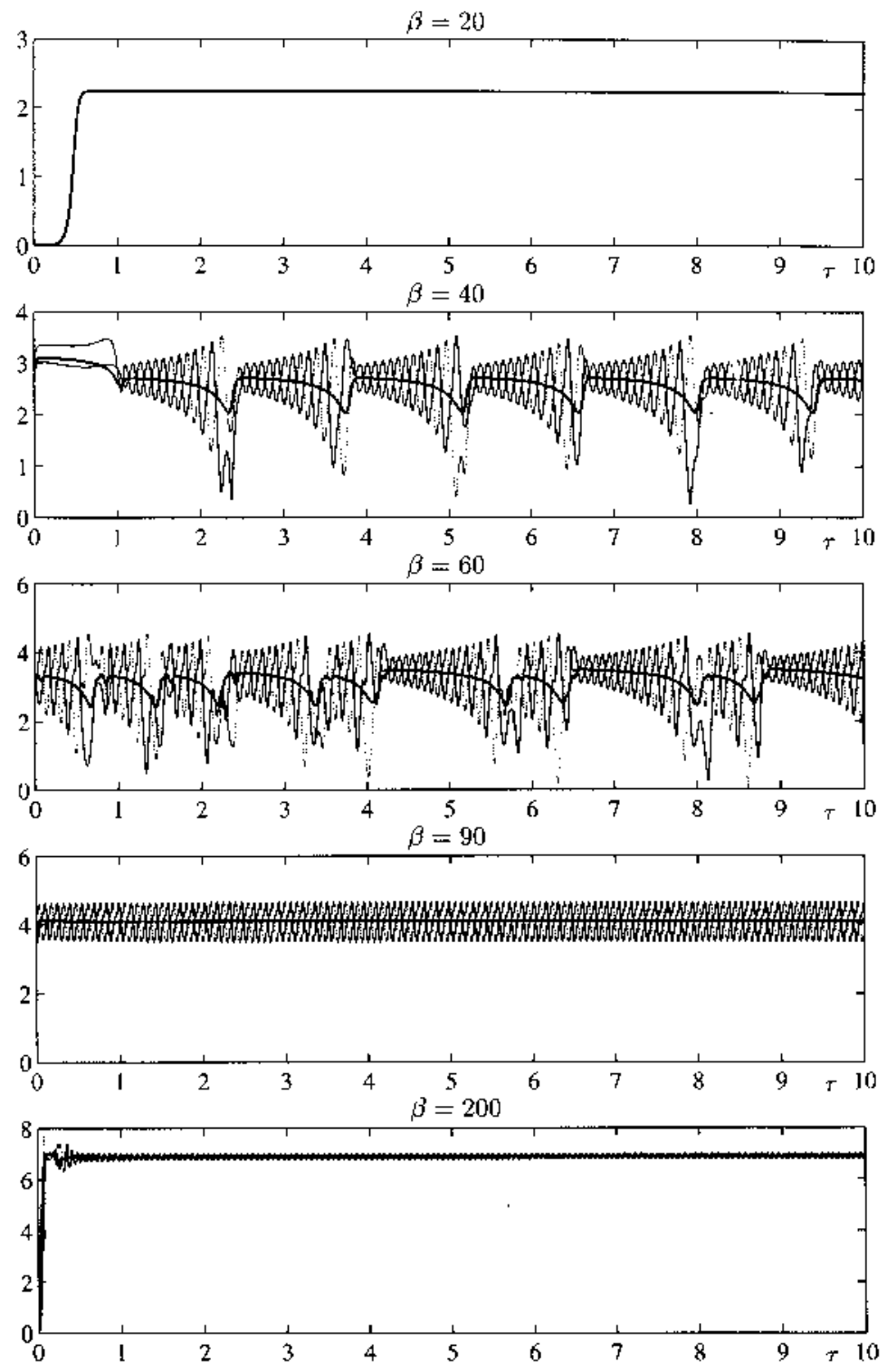

Figure 5. As in figure 3 but for $\rho=100, \alpha_{1}=3, \alpha_{2}=-1, \alpha_{3}=0, \alpha_{4}=6, \alpha_{5}^{\prime \prime}=0$ and the indicated values of $\beta$.

The solutions of $(5.3)-(5.7)$ such that

$$
\left|\varepsilon Y_{\xi \xi}\right| \ll 1 \quad \text { and } \quad\left|\varepsilon Z_{\xi \xi}\right| \ll 1
$$

for all $T \geqslant 0 . T \sim 1$. depend only on the spatial scale $\xi \sim 1$ and may be obtained by seeking for an expansion of the form

$Y=Y_{0}(\xi, T)+\varepsilon Y_{1}(\xi, T)+\cdots \quad Z=Z_{0}(\xi, T)+\varepsilon Z_{1}(\xi, T)+\cdots$

where the leading order terms are readily found to be given by the hyperbolic system

$Y_{0 T}=Y_{0 \xi}+Y_{0}\left(\lambda-\left(1+\mathrm{i} \alpha_{3}\right)\left|Y_{0}\right|^{2}-\left(\alpha_{1}+\mathrm{i} \alpha_{4}\right)\left|Z_{0}\right|^{2}\right)$ 


$$
\begin{aligned}
& Z_{0 T}=-Z_{0 \xi}+Z_{0}\left(\lambda-\left(1+\mathrm{i} \alpha_{3}\right)\left|Z_{0}\right|^{2}-\left(\alpha_{1}+\mathrm{i} \alpha_{4}\right)\left|Y_{0}\right|^{2}\right) \quad \text { in }-1 / 2<\xi<1 / 2 \\
& Z_{0}=\rho \mathrm{e}^{\mid \alpha_{s}} Y_{0} \quad \text { at } \xi=-1 / 2 \quad Y_{0}=\rho \mathrm{e}^{\mid \alpha_{s}} Z_{0} \quad \text { at } \xi=1 / 2 .
\end{aligned}
$$

That system will be considered in section 5.2. Here we only point out that althougth the boundary conditions (5.6), (5.7) cannot be imposed on $Y_{0}$ and $Z_{0}$, they are precisely such that every solution of $(5.10)$, (5.11) satisfies them for all $T \geqslant 0$ provided that these conditions hold at $T=0$. Then no boundary layer is necessary near $\xi= \pm 1 / 2$ to impose (5.6). (5.7). This fact is essential for the consistency of our weakly nonlinear description. If the boundary conditions (5.6), (5.7) were not satisfied by the solutions of (5.10). (5.11), then two boundary layers would be necessary near $\xi= \pm 1 / 2$; the characteristic size of those layers should be such that $\left|Y_{\xi \xi}\right| \sim\left|Y_{\xi}\right|$ and $\left|Z_{\xi \xi}\right| \sim\left|Z_{\xi}\right|$. i.e. $|\xi \pm 1 / 2| \sim \varepsilon$, or $|x \pm L / 2| \sim 1$; then the boundary layers would be precisely those considered in section 2.2 (where (5.3), (5.4) do not apply, see section 2.2) that allowed us to obtain the boundary conditions (5.6), (5.7).

Notice that conditions (5.8) mean that the smaller spatial scales are large as compared to $\varepsilon^{1 / 2}=L^{-1 / 2}$ in the $\xi$-variable (or to $L \varepsilon^{1 / 2}=L^{1 / 2}$ in the original $x$-variable). If condition (5.8) does not hold, then $Y$ and $Z$ depend on smaller spatial scales (than those of the size of the domain. $\xi \sim 1$ or $x \sim L$ ) and the approximation (5.9) breaks down. That case will be considered elsewhere [44]. Here. we only derive sufficient conditions for (5.8) to hold in the time scale $T \sim \mathrm{I}$ provided that it holds at $T=0$. To do that. in section 5. I we shall analyse the linear evolution of an small perturbation (added to a solution of the type (5.9)) not satisfying (5.8). If all such perturbations are damped out exponentially as $T \rightarrow \infty$, then we conclude that the approximation (5.9) makes sense for appropriate initial conditions.

The size of the small scales mentioned above is of the order of $L^{1 / 2}$ in the original $x$-variable; then these scales are in between that of the basic smallest scale, $x \sim 1$, and the size of the domain, $x \sim L$, and will be called intermediate scales in the remainder of this paper.

\subsection{Linear evolution of incipient intermediate scales}

In order to analyse the linear evolution of small perturbations containing intermediate scales (i.e. not satisfying (5.8)) around a solution without those scales (i.e. satisfying (5.8)), we first anticipate that those scales are such that $\varepsilon\left|Y_{\xi \xi}\right| \sim|Y| \sim 1, \varepsilon\left|Z_{\xi \xi}\right| \sim|Z| \sim 1,\left|Y_{\xi}\right| \sim\left|Y_{T}\right|$ and $\left|Z_{\xi}\right| \sim\left|Z_{T}\right|$. Then we introduce the new short space and time variables

$$
\eta=\varepsilon^{-1 / 2} \xi \quad \sigma=\varepsilon^{-1 / 2} T
$$

and consider a solution of $(5.3)-(5.7)$ of the form

$$
\begin{aligned}
& Y(\xi, T ; \eta, \sigma)=\left[Y_{0}(\xi, T)+\cdots\right][1+y(\xi, T ; \eta, \sigma)+\cdots] \\
& Z(\xi, T ; \eta, \sigma)=\left[Z_{0}(\xi, T)+\cdots\right][1+z(\xi, T ; \eta, \sigma)+\cdots]
\end{aligned}
$$

where $Y_{0}$ and $Z_{0}$ is a given solution of $(5.10)-(5.12)$ and

$$
\varepsilon \ll|y| \ll 1 \quad \varepsilon \ll|z| \ll 1 .
$$

By inserting (5.14) into (5.3)-(5.7) and taking into account (5.13) and (5.15), we get the following linear problem giving the evolution of $y$ and $z$ :

$$
\begin{gathered}
y_{\sigma}-y_{\eta}=\varepsilon^{1 / 2}\left[-y_{T}+y_{\xi}+\left(1+\mathbf{i} \alpha_{2}\right) y_{m}-\left(1+\mathbf{i} \alpha_{3}\right)\left|Y_{0}\right|^{2}(y+\bar{y})\right. \\
\left.-\left(\alpha_{1}+\mathrm{i} \alpha_{4}\right)\left|Z_{j}\right|^{2}(z+\bar{z})+\text { HOT }\right]
\end{gathered}
$$




$$
\begin{gathered}
z_{\sigma}+z_{\eta}=\varepsilon^{1 / 2}\left[-z_{T}-z_{\xi}+\left(1+\mathrm{i} \alpha_{2}\right) z_{\eta \eta}-\left(1+\mathrm{i} \alpha_{3}\right)\left|Z_{0}\right|^{2}(z+\bar{z})\right. \\
\left.-\left(\alpha_{1}+\mathrm{i} \alpha_{4}\right)\left|Y_{\omega}\right|^{2}(y+\bar{y})+\text { HOT }\right]
\end{gathered}
$$

in $-1 / 2<\xi<1 / 2,-\varepsilon^{-1 / 2} / 2<\eta<\varepsilon^{1 / 2} / 2$, with boundary conditions

$z=y+\varepsilon$ HOT

$y_{\eta}+z_{\eta}=-\varepsilon^{1 / 2}\left[y_{\xi}+z_{\xi} \pm \rho^{-2 \xi}\left(\rho^{2}-1\right)\left(1-\alpha_{1}+\mathbf{i}\left(\alpha_{3}-\alpha_{4}\right)\right)\left|Y_{0}\right|^{2}(y+\bar{y})+\right.$ HOT $]$

at $\xi= \pm 1 / 2 . \eta= \pm \varepsilon^{-1 / 2} / 2$. where HOT $=\mathrm{O}\left(\varepsilon+|y|^{2}+|z|^{2}\right)$. In fact. only the first pair of boundary conditions (5.18) will be imposed below; the second pair is readily seen to hold, up to the order $\varepsilon^{1 / 2}$, as a consequence of the first pair and (5.16), (5.17) (notice that $y_{m}=z_{m}$ to the leading order at the boundaries, see (5.19) and (5.20) below). The solution of the complex linear problem ( 5.16$)-(5.18)$ may be written as

$y=\int_{0}^{\infty}\left[y_{k}^{+}(\xi, T) \mathrm{e}^{i k(\eta+\sigma)}+y_{k}^{-}(\xi, T) \mathrm{e}^{-i k(\eta+\sigma)}\right] \mathrm{d} k+\varepsilon^{1 / 2} y_{1}(\xi, T ; \eta, \sigma)+\cdots$

$z=\int_{0}^{\infty}\left[z_{k}^{+}(\xi, T) \mathrm{e}^{\mathrm{i} k(\eta-\sigma)}+z_{k}^{-}(\xi, T) \mathrm{e}^{-i k(\eta-\sigma)}\right] \mathrm{d} k+\varepsilon^{1 / 2} z_{1}(\xi, T ; \eta, \sigma)+\cdots$

where $v_{1}$ and $z_{1}$ satisfy

$$
\begin{aligned}
y_{1 \sigma}-y_{1_{\eta}}= & \int_{0}^{\infty}\left[-y_{k T}^{+}+y_{k \xi}^{+}-k^{2}\left(1+\mathrm{i} \alpha_{2}\right) y_{k}^{+}-\left(\mathbf{I}+\mathrm{i} \alpha_{3}\right)\left|Y_{0}\right|^{2}\left(y_{k}^{+}+\bar{y}_{k}^{-}\right)\right] \mathrm{e}^{\mathrm{i} k(\eta+\sigma)} \mathrm{d} k \\
& +\int_{0}^{\infty}\left[-y_{k T}^{-}+y_{k \xi}^{-}-k^{2}\left(1+\mathrm{i} \alpha_{2}\right) y_{k}^{-}-\left(1+\mathrm{i} \alpha_{3}\right)\left|Y_{0}\right|^{2}\left(y_{k}^{-}+\bar{y}_{k}^{+}\right)\right] \mathrm{e}^{-\mathrm{i} k(\eta+\sigma)} \mathrm{d} k \\
& +\int_{0}^{\infty} \mathrm{NRT} \mathrm{d} k \\
z_{1 \sigma}+z_{1 \eta}= & \int_{0}^{\infty}\left[-z_{k T}^{+}-z_{k \xi}^{+}-k^{2}\left(\mathbf{I}+\mathrm{i} \alpha_{2}\right) z_{k}^{+}-\left(1+\mathrm{i} \alpha_{3}\right)\left|Z_{0}\right|^{2}\left(z_{k}^{+}+\bar{z}_{k}^{-}\right)\right] \mathrm{e}^{\mathrm{i} k(\eta-\sigma)} \mathrm{d} k \\
& +\int_{0}^{\infty}\left[-z_{k T}^{-}-z_{k \xi}^{-}-k^{2}\left(1+\mathrm{i} \alpha_{2}\right) z_{k}^{-}-\left(\mathbf{1}+\mathrm{i} \alpha_{3}\right)\left|Z_{0}\right|^{2}\left(z_{k}^{-}+\bar{z}_{k}^{+}\right)\right] \mathrm{e}^{-i k(\eta-\sigma)} \mathrm{d} k \\
& +\int_{0}^{\infty} \mathrm{NRT} \mathrm{d} k .
\end{aligned}
$$

Here overbars and NRT stand for the complex conjugate and non-resonant terms (i.e. terms not proportional to $\exp [ \pm \mathrm{i} k(\eta-\sigma)]$ and to $\exp [ \pm \mathrm{i} k(\eta+\sigma)]$ in (5.21) and (5.22), respectively). Notice that $y_{k}^{\mp}$ and $z_{k}^{\mp}$ account for the linear evolution of the intermediate scale with a wavenumber equal to $k$. By eliminating secular terms in (5.21) and (5.22) (i.e. by requiring $y_{1}$ and $z_{1}$ to be bounded in the time scale $\sigma \sim 1$ ) we get. for all $k>0$,

$y_{k T}^{ \pm}=y_{k \xi}^{ \pm}-k^{2}\left(1+\mathbf{i} \alpha_{2}\right) y_{k}^{ \pm}-\left(\mathbf{I}+\mathrm{i} \alpha_{3}\right)\left|Y_{i}\right|^{2}\left(y_{k}^{ \pm}+\bar{y}_{k}^{\mp}\right)$

$z_{k T}^{ \pm}=-z_{k \xi}^{ \pm}-k^{2}\left(1+\mathbf{i} \alpha_{2}\right) z_{k}^{ \pm}-\left(1+\mathbf{i} \alpha_{3}\right)\left|Z_{0}\right|^{2}\left(z_{k}^{ \pm}+z_{k}^{\mp}\right) \quad$ in $-1 / 2<\xi<1 / 2$

while the first boundary conditions (5.18) yield

$$
v_{k}^{+}=z_{k}^{-} \mathrm{e}^{\mp \mathrm{i} r_{k}} \quad v_{k}^{-}=z_{k}^{+} \mathrm{e}^{ \pm \mathrm{i} \mathrm{r}_{k}} \quad \text { at } \xi= \pm 1 / 2
$$

where $\nu_{k}=2 \pi \operatorname{fract}(k \sqrt{L} / 2 \pi)$. with fract $(x) \equiv$ fractional part of $x$.

In order to obtain equations and boundary conditions with real coefficients (and to eliminate the phase $v_{k}$ ) we may use the new variables

$$
\begin{array}{ll}
u_{k}^{+}=\left(y_{k}^{+}+\bar{y}_{k}^{-}\right) \mathrm{e}^{\mathrm{i} v_{k}(\xi+T)} & v_{k}^{+}=\mathrm{i}\left(\bar{y}_{k}^{-}-y_{k}^{+}\right) \mathrm{e}^{\mathrm{i} v_{k}(\xi+T)} \\
u_{k}^{-}=\left(\bar{z}_{k}^{+}+z_{k}^{-}\right) \mathrm{e}^{\mathrm{i} \nu_{k}(-\xi+T)} & v_{k}^{-}=\mathbf{i}\left(\bar{z}_{k}^{+}-z_{k}^{-}\right) \mathrm{e}^{\nu_{k}(-\xi+T)}
\end{array}
$$


to write $(5.23)-(5.25)$ as

$$
\begin{aligned}
& u_{k T}^{+}-u_{k \xi}^{+}=-\left(k^{2}+2\left|Y_{0}\right|^{2}\right) u_{k}^{+}+\alpha_{2} k^{2} v_{k}^{+} \\
& v_{k T}^{+}-v_{k \xi}^{+}=-\left(\alpha_{2} k^{2}+2 \alpha_{3}\left|Y_{j}\right|^{2}\right) u_{k}^{+}-k^{2} v_{k}^{+} \\
& u_{k T}^{-}+u_{k \xi}^{-}=-\left(k^{2}+2\left|Z_{0}\right|^{2}\right) u_{k}^{-}+\alpha_{2} k^{2} v_{k}^{-} \\
& v_{k T}^{-}+v_{k \xi}^{-}=-\left(\alpha_{2} k^{2}+2 \alpha_{3}\left|Z_{0}\right|^{2}\right) u_{k}^{-}-k^{2} v_{k}^{-} \quad-1 / 2<\xi<1 / 2 \\
& u_{k}^{+}=u_{k}^{-} \quad v_{k}^{+}=v_{k}^{-} \quad \text { at } \xi= \pm 1 / 2 \text {. }
\end{aligned}
$$

Now, by collecting the results of the formal analysis above we may state the following properties concerning the stability of the solutions of $(5.10)-(5.12)$ under small perturbations containing the intermediate scales. Take a solution of $(5.10)-(5.12), Y_{0}=Y_{0}(\xi, T)$ and $Z_{0}=Z_{0}(\xi, T)$.

Property 1. If for each $k>0$, every solution of (5.27)-(5.31) is such that

$$
u_{k}^{ \pm} \rightarrow 0 \quad \text { and } \quad v_{k}^{ \pm} \rightarrow 0 \text { exponentially as } T \rightarrow \infty
$$

then any small perturbation of $\left(Y_{0}, Z_{0}\right)$ containing the intermediate scales is damped out exponentially as $T \rightarrow \infty$. In this case we conclude that $\left(Y_{0}, Z_{0}\right)$ is a good approximation of a solution of $(5.3)-(5.7)$ in the time scale $T \sim 1$.

Property 2. If, for some $k>0$ there is a solution of (5.27)-(5.31) that diverges exponentially as $T \rightarrow \infty$, i.e. such that there is a sequence $\left\{\left(\xi_{n}, T_{n}\right)\right\}$ satisfying $-1 / 2 \leqslant$ $\xi_{n} \leqslant 1 / 2, T_{n} \geqslant n$ for all $n$ and

$\left|u_{k}^{+}\left(\xi_{n}, T_{n}\right)\right|+\left|u_{k}^{-}\left(\xi_{n}, T_{n}\right)\right|+\left|v_{k}^{+}\left(\xi_{n}, T_{n}\right)\right|+\left|v_{k}^{-}\left(\xi_{n}, T_{n}\right)\right| \rightarrow \infty$ exponentially as $n \rightarrow \infty$.

Then there are small perturbations of $\left(Y_{0}, Z_{0}\right)$, containing the intermediate scales, that do not remain small for all $T>0$. In this case, we conclude that $\left(Y_{0}, Z_{0}\right)$ cannot be considered a good approximation of a solution of (5.3)-(5.7) in the time scale $T \sim 1$.

Notice that although $u_{k}^{ \pm}$and $v_{k}^{ \pm}$are complex, their real and imaginary parts also satisfy (5.27)-(5.31) (because the coefficients in the equations and boundary conditions are real). Then we may consider $u_{k}^{ \pm}$and $v_{k}^{ \pm}$as real functions when analysing whether they satisfy one of the conditions (5.32) or (5.33).

The linear problem (5.27)-(5.31) is considered in appendix B, where sufficient conditions are obtained for properties 1 and 2 to apply (see theorem B.5 at the end of the appendix). Several remarks concerning the results in this section and those in appendix B are in order:

(a) The problem (5.27)-(5.31), giving the linear evolution of the intermediate scales, depends explicitly only on $\alpha_{2}$ and $\alpha_{3}$ (of course there is an implicit dependence on $\rho, \lambda$ and $\alpha_{1}$ through the functions $\left|Y_{0}\right|$ and $\left.\left|Z_{i}\right|\right)$. 

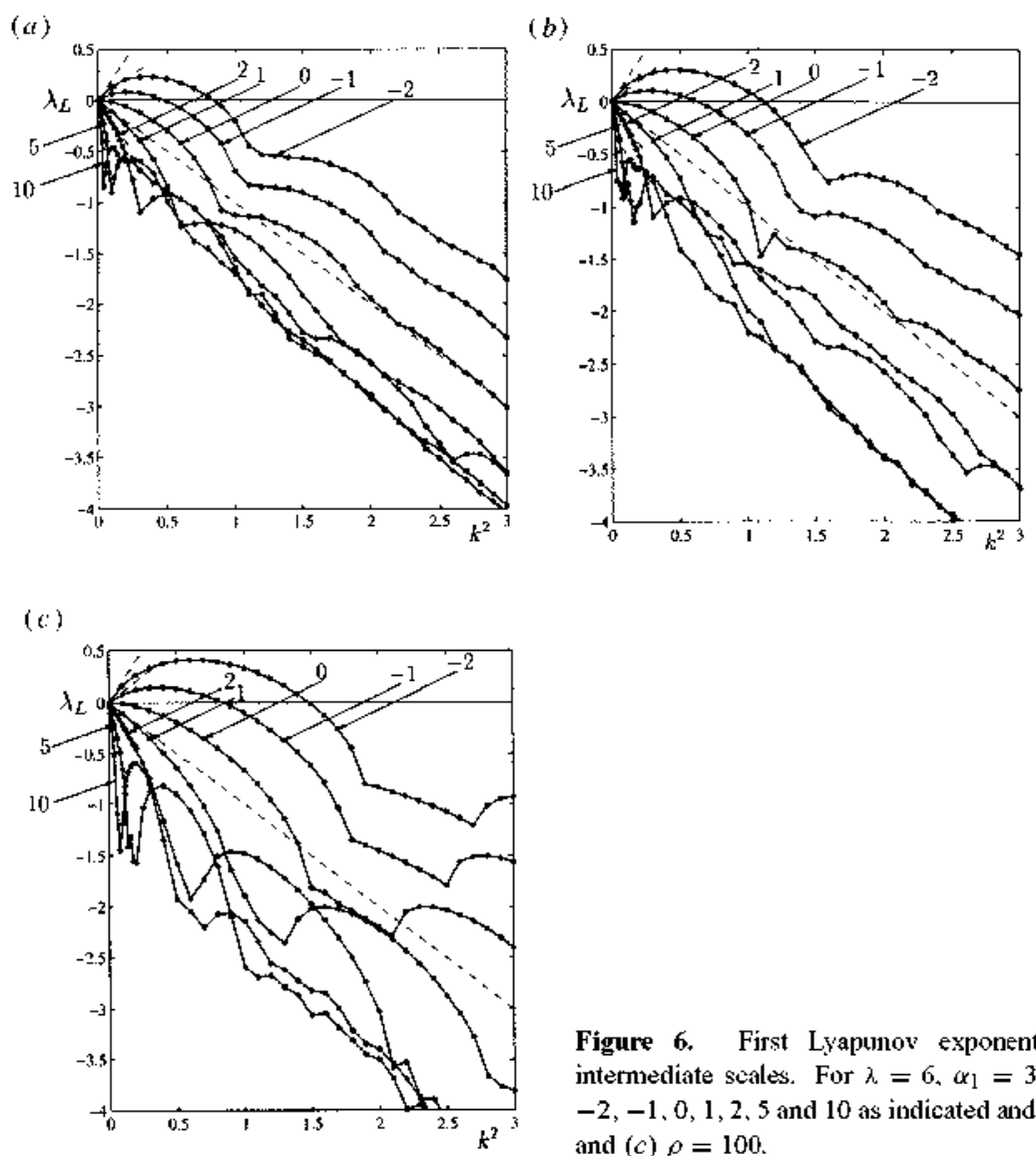

Figure 6. First Lyapunov exponent associated with the intermediate seales. For $\lambda=6, \alpha_{1}=3, \alpha_{2}=1,1+\alpha_{2} \alpha_{3}=$ $-2,-1,0,1,2,5$ and 10 as indicated and $(a) \rho=.01,(b) \rho=1$ and $(c) \rho=100$.

(b) Theorem B.5 in appendix B gives sufficient conditions, for stability or instability under perturbations dependent on the intermediate scales. that do not depend on the basic solution $\left|Y_{0}\right|,\left|Z_{0}\right|$. The following conjecture was suggested by several numerical calculations (such as those in figure 7): 'Property $\mathbf{I}$ applies if $1+\alpha_{2} \alpha_{3}>0$. and property 2 does if $1+\alpha_{2} \alpha_{3}<0$. Unfortunately. we were able to prove the conjecture only in the case where $\left|Y_{0}\right|$ and $\left|Z_{0}\right|$ are spatially uniform and constant (this requires the boundaries to be perfectly reflecting. i.e. $\rho$ to be equal to one), that is considered in lemma B.I. in appendix $B$. If this conjecture were true the appearance of the intermediate scales could be anticipated very easily. At the moment, if $\alpha_{2} \alpha_{3} \geqslant 1$, in order to conclude that the intermediate scales do not come into play, one must integrate (5.27)-(5.31) for many values of $k$ in the interval $0<k \leqslant\left(\mu F^{*}\right)^{1 / 2}$ ( $\mu$ and $F^{*}$ as in lemma B.3, in appendix B) to ensure that the intermediate scales with these wavelengths are damped out as $T \rightarrow \infty$ (if $-1<\alpha_{2} \alpha_{3}<1$ then the result is true for all $k$, according to lemma B.2, in appendix B). As an example, the maximum Lyapunov exponent associated with the large time behaviour of the system $(5.27)-(5.31)$, defined as

$\lambda_{L}=\limsup \log \int_{-1 / 2}^{1 / 2}\left(\left|u_{k}^{+}\right|^{2}+\left|v_{k}^{+}\right|^{2}+\left|u_{k}^{-}\right|^{2}+\left|v_{k}^{-}\right|^{2}\right) \mathrm{d} \xi /(2 T) \quad$ as $T \rightarrow \infty$

is plotted in figure 6 for a generic solution of (5.27)-(5.31), in terms of the re-scaled intermediate scales wavenumber $k^{2}$. for $\alpha_{1}=3, \lambda=6, \alpha_{2}=-1$ and the indicated values 

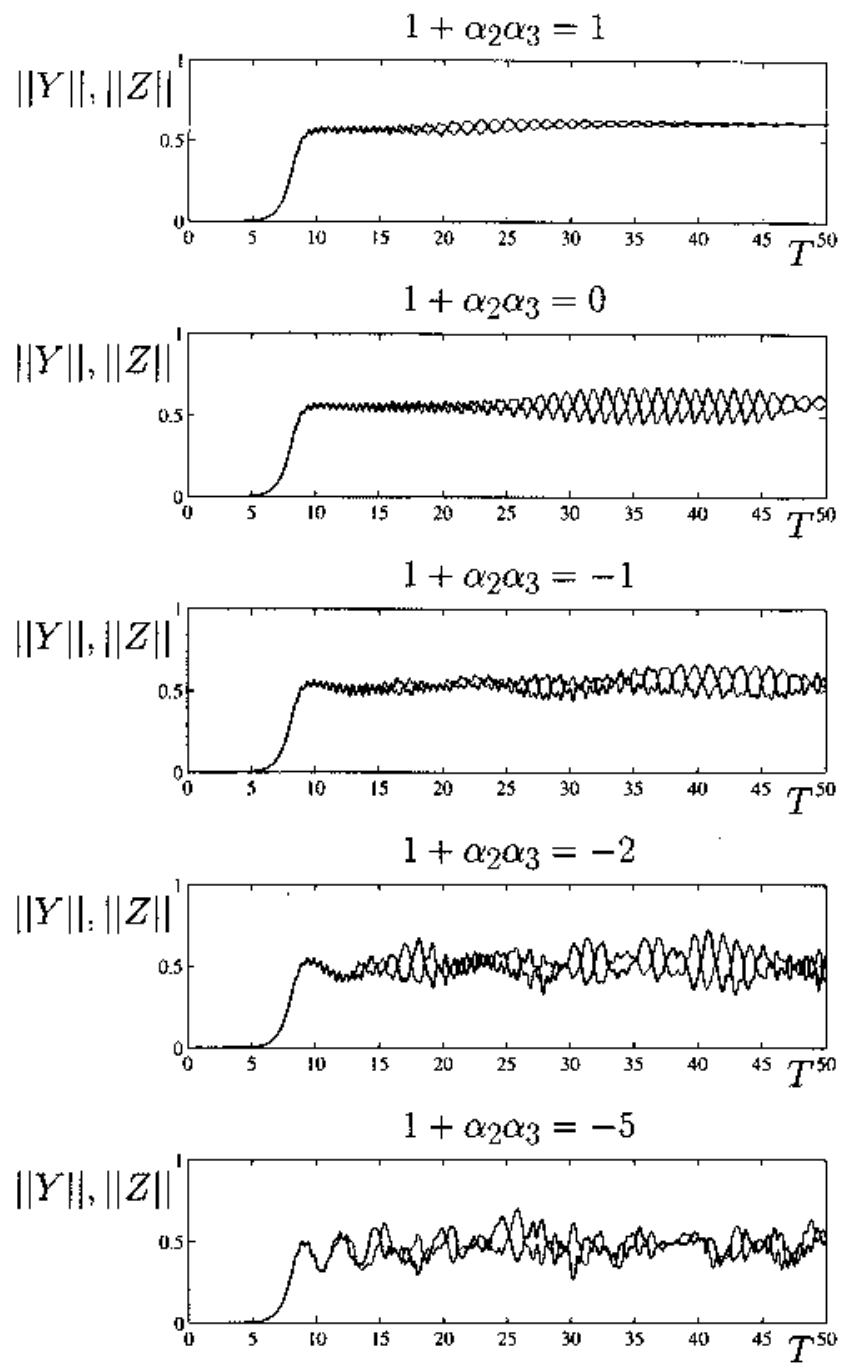

Solution of Hyperbolic Eqs.

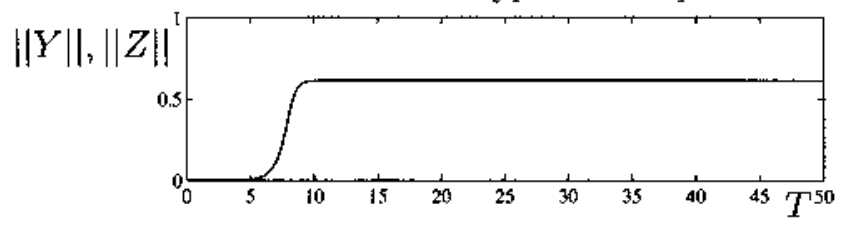

Figure 7. $\|Y\|$ (thick curve) and $\|Z\|$ (thin curve) versus $T$, as obtained by numerical integration of $(5.3)-(5.7)$ for $\varepsilon \equiv L^{-1}=10^{-2}, \beta=1, \lambda=1.5, \alpha_{1}=3, \alpha_{2}=-1, \alpha_{4}=\alpha_{5}=0$ and $1+\alpha_{2} \alpha_{3}=1,0,-1,-2$ and -5 , and the corresponding solution of the hyperbolic systen $(5.10)-(5.12)$.

of $\alpha_{3}$ and $\rho$. Notice that if $\alpha_{2} \alpha_{3} \geqslant 1$ then the maximum Lyapunov exponent is always negative (i.e. all solutions of (5.27)-(5.31) are damped out exponentially as $T \rightarrow \infty$ ): in fact, it is always smaller than $-k^{2}$ (the broken line in figure 6 is precisely $\lambda_{L}=-k^{2}$ ). 

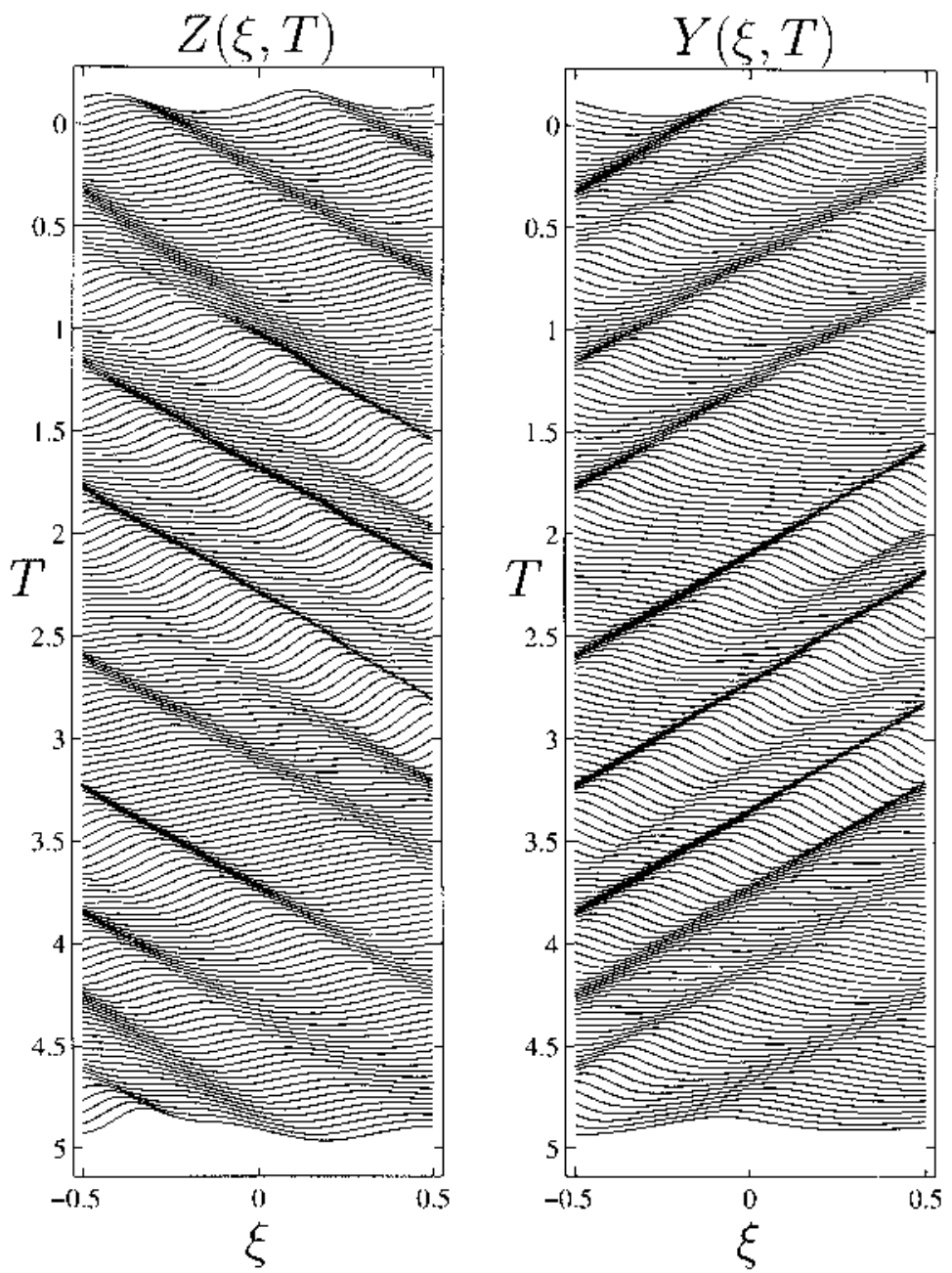

Figure 8. Temporal evolution of the spatial profiles of $|Y|$ and $|Z|$ for the case $1+\alpha_{2} \alpha_{3}=-5$ in figure 7 .

When the intermediate scales come into play, no simple asymptotic sub-model of (5.3)(5.7) can be obtained (except for some particular limits. such as that considered in remark $\mathrm{C}$ below) and the truncated (terms of order $O\left(\varepsilon^{2}\right)$ are ignored) system (5.3)-(5.7) must be considered. This system has been numerically integrated by means of a finite differences scheme with $\varepsilon=L^{-1}=10^{-2}$, for $\rho=1 . \lambda=1.5, \alpha_{1}=3, \alpha_{2}=-1, \alpha_{4}=\alpha_{5}=0$ and $1+\alpha_{2} \alpha_{3}=1,0,-1,-2$ and -5 ; the spatial $L_{2}$-norms of the amplitudes $Y$ and $Z$ are given, in terms of $T$, in figure 7. For $\mathbf{1}+\alpha_{2} \alpha_{3}=1$ and $0, Y$ and $Z$ evolves to a constant steady state such that $|Y| \equiv|Z| \equiv \sqrt{3 / 8}$, that corresponds precisely to the large time behaviour of the solution of the hyperbolic approximation, $Y_{0}, Z_{0}$, given by $(5.10)-(5.12)$; the solution of (5.10)-(5.12) with the same initial conditions as those applied to (4.5)-(4.7), is also given in figure 7 for comparison. Notice that $Y \simeq Y_{0}$ and $Z \simeq Z_{0}$ for all $T$ in the first two cases of figure 7 (that is. when the intermediate scales are inhibited); in the last three cases, instead, both evolutions are completely different due to the intermediate scales. A $\xi$ against 
$T$ diagram of $Y$ and $Z$ for the case $1+\alpha_{2} \alpha_{3}=-5$ in figure 7 is given in figure 8 . Notice that the profiles exhibit scales that are intermediate, between the basic-wavelength scale (of the order of $\varepsilon=10^{-2}$ after the re-scaling above) and the size of the domain.

(c) If $\left|Y_{i}\right|$ and $\left|Z_{i j}\right|$ are uniformly bounded below by a strictly positive constant as $T \rightarrow \infty$ then, according to theorem B.5 in appendix B, there is a qualitative change in the behaviour of the intermediate scales as $1+\alpha_{2} \alpha_{3}$ changes from positive to negative values. If $0 \leqslant-1-\alpha_{2} \alpha_{3} \ll 1$ then one should be able to obtain a canonical nonlinear problem giving the weakly nonlinear evolution of the intermediate scales. This would be of great interest in understanding the role of those scales when they are not damped out.

\subsection{Some results concerning the nonlinear wave sustem}

Here we obtain some basic properties of the hyperbolic wave system (5.10)-(5.12) and give some numerical results.

First we introduce the real variables $u>0, v>0, \theta$ and $\varphi$, defined as

$$
Y_{0}=\sqrt{u} \exp (\mathbf{i} \theta) \quad Z_{0}=\sqrt{v} \exp (\mathrm{i} \varphi)
$$

to write $(5.10)-(5.12)$ as

$$
\begin{aligned}
& u_{T}=u_{\xi}+2 u\left(\lambda-u-\alpha_{1} v\right) \quad v_{T}=-v_{\xi}+2 v\left(\lambda-v-\alpha_{1} u\right) \quad \text { in }-1 / 2<\xi<1 / 2 \\
& v=\rho^{2} u \quad \text { at } \xi=-1 / 2 \quad u=\rho^{2} v \quad \text { at } \xi=1 / 2 \\
& \theta_{T}=\theta_{\xi}-\alpha_{3} u-\alpha_{4} v \quad \varphi_{T}=-\varphi_{\xi}-\alpha_{3} v-\alpha_{4} u \quad \text { in }-1 / 2<\xi<1 / 2 \\
& \varphi=\theta+\alpha_{5} \quad \text { at } \xi=-1 / 2 \quad \theta=\varphi+\alpha_{5} \quad \text { at } \xi=1 / 2 .
\end{aligned}
$$

Notice that the problem (5.35), (5.36), giving $u$ and $"$, is decoupled from (5.37), (5.38). Once $u$ and $v$ have been calculated from (5.35), (5.36), with appropriate initial conditions, the solution of the linear problem (5.37), (5.38) may be written as $\theta=\theta_{p}+\theta_{H}, \varphi=\varphi_{p}+\varphi_{H}$, where $\left(\theta_{p}, \varphi_{p}\right)$ is any particular solution of $(5.37),(5.38)$, while $\left(\theta_{H}, \varphi_{H}\right)$ is the general solution of the homogeneous problem $\theta_{H T}-\theta_{H \xi}=\varphi_{H T}+\varphi_{H \xi}=0$ in $-1 / 2<\xi<1 / 2$, $\theta_{H}=\varphi_{H}$ at $\xi= \pm 1 / 2$. Thus $\theta_{H}$ and $\varphi_{H}$ depend on arbitrary functions that evolve in a slower time scale. A nonlinear problem may be obtained for the evolution of $\theta_{H}$ and $\varphi_{H}$ in the slower time scale, that exhibits chaotic solutions for appropriate values of the parameters (see [44]): those chaotic solutions correspond to phase turbulence. For the sake of brevity we do not pursue this matter any further in this paper. Instead we shall focus on the main problem (5.35), (5.36). that gives the (squares of the) amplitudes of the counterpropagating waves; as we shall explain below, that problem also exhibits chaotic solutions.

Global existence and uniqueness properties of the problem (5.35), (5.36), with appropriate initial conditions,

$$
u=u_{0}(\xi)>0 \quad v=v_{0}(\xi)>0 \quad \text { at } T=0
$$

and global stability properties of the steady states are considered in [43] where, in particular, the following properties are proved:

(a) If $\alpha_{1}<-1$ (resp., $\alpha_{1}=-1$ ) then (5.35). (5.36). (5.39) possess solutions that diverge in finite time (resp., as $T \rightarrow \infty$ ), i.e.

$$
\max \{|u(\xi, T)|+|v(\xi, T)| \quad 1 / 2 \leqslant \xi \leqslant 1 / 2\} \rightarrow \infty
$$

as $T \rightarrow T_{0}<\infty$ (resp., as $T \rightarrow \infty$ ). Recall that the same property was true for the averaged Ginzburg-Landau equation, that applied in the first regime (see section 4.2); again, this result means that if $\alpha_{1}<-1$ (resp., $\alpha_{1}=-1$ ) then the analysis of the large-time dynamics of 


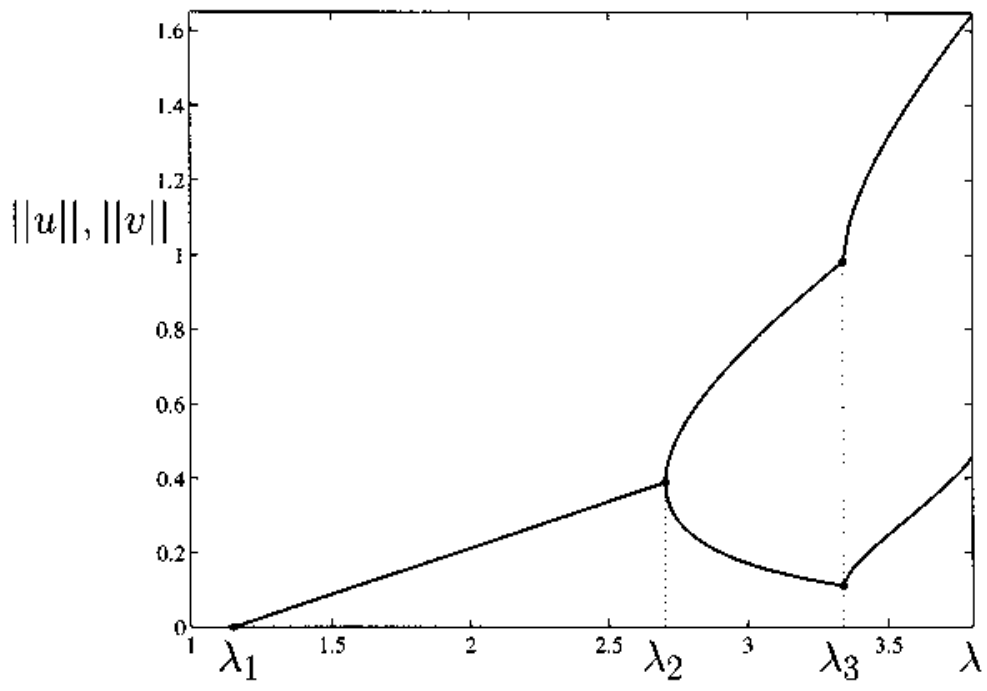

Figure 9. Bifurcation diagram of the nonlinear wave system (5.35). (5.36) for $\rho=0.1$ and $\alpha_{1}=3$.

the underlying physical system requires that we consider the fully-nonlinear model (resp., requires that we consider higher order terms in the amplitude equations).

(b) If $\alpha_{1}>-1$ then every solution of (5.35), (5.36), (5.39) is uniformly bounded in $-1 / 2 \leqslant \xi \leqslant 1 / 2,0 \leqslant T<\infty$. If, in addition $\lambda \leqslant-\log \rho$ then $|u|+|v| \rightarrow 0$, uniformly in $-1 / 2 \leqslant \xi \leqslant 1 / 2$, as $T \rightarrow \infty$.

(c) If $-1<\alpha_{1}<1$ and $\lambda>-\log \rho$ then (5.35), (5.36) has a unique steady state, $\left(u_{s}, v_{s}\right)$. such that $u_{s}>0$ and $v_{s}>0$ in $-1 / 2 \leqslant \xi \leqslant 1 / 2$, and every solution of (5.35), (5.36), (5.39) is such that $u \rightarrow u_{s}$ and $v \rightarrow v_{s}$. uniformly in $-1 / 2 \leqslant \xi \leqslant 1 / 2$. as $T \rightarrow \infty$.

Global stability properties are obtained in [43] by means of comparison methods that do not apply if $\alpha_{1} \geqslant I$ and $\lambda>-\log \rho$. This case is considered in [44]. where continuation methods, standard dynamical systems techniques and numerical integration of (5.35), (5.36) are systematically used. As an example of the results obtained in [44], the attractors (as $T \rightarrow \infty)$ of (5.35), (5.36) are plotted in figures 9 and 10 in terms of the bifurcation parameter $\lambda$ for the particular case $\alpha_{1}=3$ and $\rho^{2}=0.1$. As shown in figure 9 , at $\lambda=\lambda_{1} \equiv-\log \rho \simeq 1.15$ there is a supercritical bifurcation from the basic steady state, $u \equiv v \equiv 0$, to a new branch of symmetric steady states (i.e. invariant under the symmetry $u \rightarrow v, v \rightarrow u, x \rightarrow-x$ ). As an example. the bifurcated steady state for $\lambda=2$ is plotted in figure $10(a)$ : when taking into account the fact that $\sqrt{u}$ and $\sqrt{v}$ are essentially the modulating amplitudes of two counterpropagating wavetrains (see $(2.13),(5.1),(5.9)$ and (5.34)), these bifurcated steady states are seen to correspond to stmmetric chevrons in the spacetime diagram of the underlying physical problem. At $\lambda=\lambda_{2} \simeq 2.70$ there is a secondary supercritical pitchfork bifurcation to non-symmetric steady states (i.e. a symmetry breaking); as an example, one of the two non-symmetric steady states at $\lambda=3$ is plotted in figure $10(b)$, and corresponds to a non-summetric chevron. At $\lambda=\lambda_{3} \simeq 3.35$ there are two super-critical Hopf bifurcations (one from each branch of non-symmetric steady states) to non-symmetric limit cycles; that for $\lambda=3.5$ is plotted in figure $10(\mathrm{c})$. Its period is $T_{0} \simeq 3.09$, and corresponds to a beating state (essentially only one of the two counterpropagating wavetrains is seen in most part of the domain, and the size of this part 

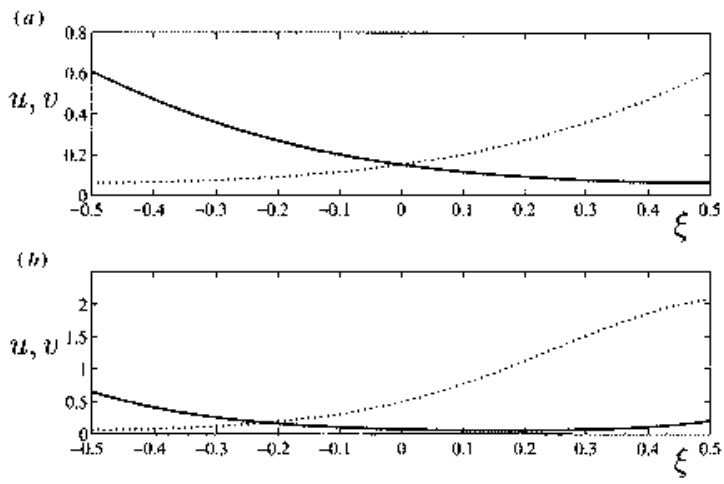

Figure 10. Aftractors as $T \rightarrow \infty$ of the nonlinear wave system for the case in figure 9 and $(a)$ $\lambda=2,(b) \lambda=3$ and $(c) \lambda=3.5$ ( $u$; solid curve and $v$ : dotted curve).

varies periodically in time; the other wave is seen only in a quite narrow region). The remaining part of the bifurcation diagram is not precisely described here for the sake of brevity. Let us just say that for larger values of $\lambda$ there is a period-doubling sequence that leads to a non-symmetric a chaotic attractor that, as $\lambda$ increases. becomes symmetric; as $\lambda$ is further increased the attractor becomes periodic through an intermittency-like process. This way of gaining symmetry through chaos is known as crisis. For higher values of $\lambda$, symmetry is lost again through a transcritical bifurcation and a second crisis takes place as $\lambda$ is further increased. See [44] for details. For other values of the parameters $\rho>0$ and $\alpha_{1}>1$. bifurcation diagrams in terms of $\lambda$ are qualitatively similar to that described above.

\section{Concluding remarks}

The amplitude equations that apply at the onset of the oscillatory instability in large domains, and the appropriate boundary conditions when the domain is finite, have been obtained for ID reaction-diffusion systems in section 2, and for more general problems in appendix A. In the generic case when the group velocity is of order unity. the amplitude equations contain terms that are not of the same order. This fact has allowed us to obtain two asymptotic submodels in the distinguished limits (3.7) and (3.8). In the first distinguished limit we have reduced the amplitude equations to an averaged Ginzburg-Landau model, that accounts for diffusion, dispersion. nonlinearity and interaction of each wavetrain with a weighted average of the counterpropagating one. That model was obtained in section 4.1, and some of its properties were briefly considered in section 4.2 ; a more complete analysis of the model will be given elsewhere [42]. In the second distinguished regime, the real amplitudes and phases associated with the complex amplitudes become decoupled in the first approximation provided that some intermediate scales (whose wavelength is large compared with the basic wavelength of the counterpropagating wavetrains, but small compared with the size of the spatial domain) are inhibited; a linear model giving the incipient evolution of the intermediate scales was obtained in section 5.1 and analysed in appendix B, where sufficient conditions were derived for the intermediate scales to be exponentially damped out for large time. When the intermediate scales are inhibited, the evolution of the real amplitudes of the counterpropagating wavetrains is given by a system of two nonlinear wave equations that account for wave propagation and nonlinear interaction, with boundary conditions accounting for linear reflection at the boundaries; that system is thoroughly 

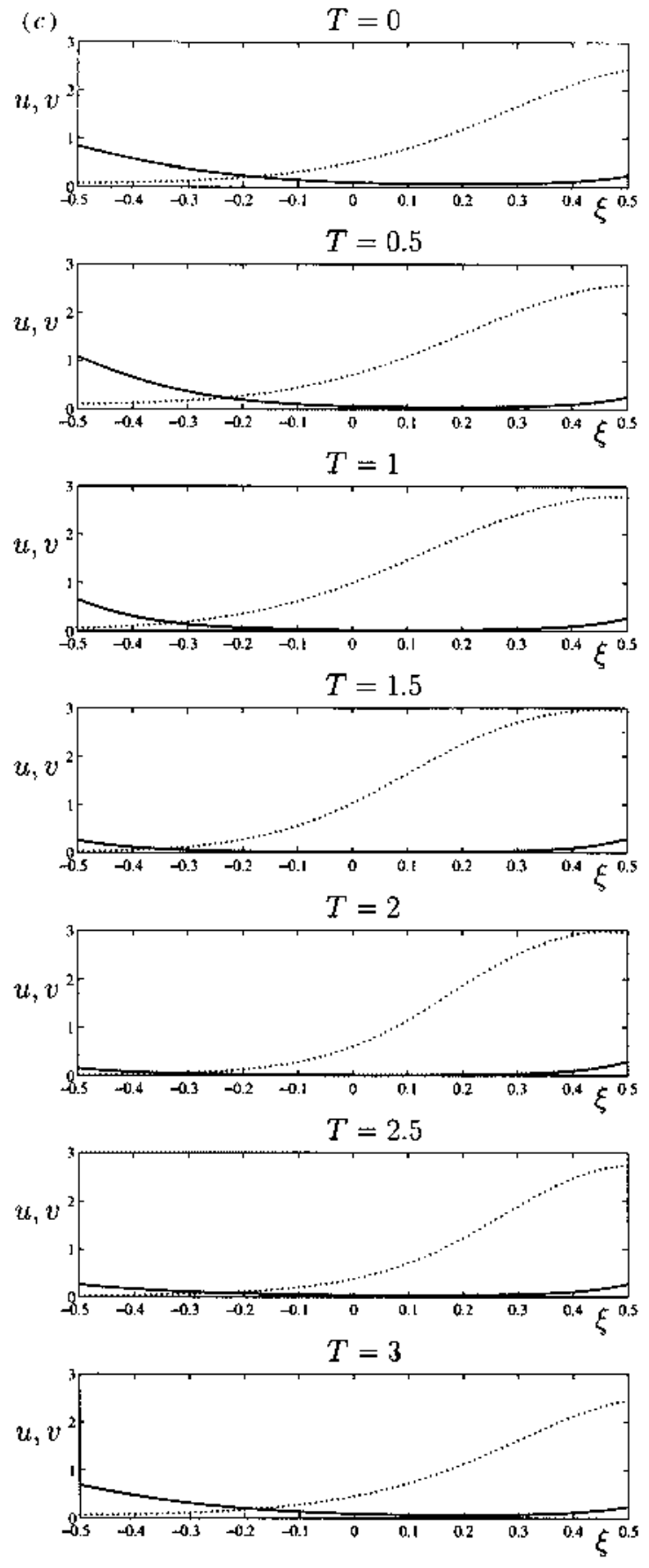

Figure 10. (Continued.)

analysed in $[43,44]$ and briefly discused in section 5.2 .

We believe that our results provide a fairly complete picture of the weakly nonlinear behaviour of physical systems near the onset of the oscillatory instability. Qualitative and quantitative comparison with experimental results in the literature $[41,44]$ are fairly good. 
Notice that our analysis predicts two main sources of complexity:

(a) The modulational instability in the first distinguished regime, and the appearance of the intermediate scales in the second distinguished limit, that come into play whenever

$$
1+\alpha_{2} \alpha_{3}<0 .
$$

(b) The presence of the walls. In the first distinguished regime, the averaged GinzburgLandau model exhibits complex behaviour if $1+\alpha_{2} \alpha_{3}>0$ but the remaining parameters are appropriate. In the second distinguished regime, the hyperbolic system exhibits chaotic behaviour if $\alpha_{1}>1$ and $\lambda$ is appropriate.

Finally, let us just mention two obvious extensions of our results, that will be considered elsewhere.

- If the governing equations are still invariant under reflection (and translation) but the boundary conditions at $x= \pm L / 2$ are not, then the amplitude equations (3.1), (3.2) still apply, but the reflection coefficient, $\rho \mathrm{e}^{\mathrm{i} \alpha_{s}}$, is not the same in both boundary conditions (3.3) and (3.4). This extension is of interest in some experiments; for example, in the TaylorCouette system, the upper end of the liquid in between of the counter-rotating cylinders is sometimes left open.

- As mentioned in section 1 , if the spatial domain has no boundaries (e.g. if it is an annulus) then the boundary conditions (1.4)-(1.6) must be replaced by $\left(1.4^{\prime}\right)$. Then the distinguished limits (3.7). (3.8) must be still considered. with $\mu_{c}=0$. In the first distinguished limit. a system of two Ginzburg-Landau equations is obtained. with nonlocal averaged coupling terms. In the second distinguished limit, the system (5.35) is still obtained, with the new boundary conditions

$u(\xi+1, T)=u(\xi, T) \quad v(\xi+1, T)=v(\xi, T) \quad$ for all $\xi \in \mathbb{R}, T \geqslant 0$.

A preliminary analysis of the asymptotic submodels has suggested that if $1+\alpha_{2} \alpha_{3}>0$ then no complexity appears for large time. That conclusion makes sense because the source of complexity (b) is now absent.

\section{Acknowledgments}

This research was supported by DGICYT and the EEC Program on Human Capital and Mobility. under grants PB-94-0416 and CHRX-CT-93-0413.

\section{Appendix A. The complete set of boundary conditions in a general case}

Here we shall obtain the boundary conditions $(1.4)-(1.6)$ in a fairly general case to show that they apply to a large variety of physical problems. For the sake of clarity we will not pursue complete generality. Instead we shall require our results to apply at least to

(a) $2 D$ and $3 D$ reaction-diffusion systems in slender strips and cylinders

Here the governing equations are

$u_{t}=D u_{x x}+D \tilde{\Delta} u+f(u, \mu) \quad$ in $-L / 2<x<L / 2 \quad y \in \Omega$

$C u+E u_{\nu}=0 \quad$ at $-L / 2<x<L / 2 \quad y \in \partial \Omega$

$C u \pm E u_{x}=0 \quad$ at $x= \pm L / 2 \quad y \in \Omega$

where $\Omega \subset \mathbb{R}^{n-1}$ ( $n=2$ or 3 ) is a bounded domain. with boundary $\partial \Omega . v$ is the outward unit normal to $\partial \Omega$ and the spatial coodinates, $\left(x_{1}, x_{2}\right)$ or $\left(x_{1}, x_{2}, x_{3}\right)$. are written as $(x, y)$, 
with $x=x_{1}$ and $y=x_{2}$ or $y=\left(x_{2}, x_{3}\right) ; u \in \mathbb{R}^{N}, \bar{\Delta}$ is the transversal Laplacian operator, $f$ is the nonlinear reaction term that depends on the control parameter $\mu$ and the $N \times N$ matrices $D, C$ and $E$ are such that the problem above, with appropriate initial conditions, is well posed (e.g. $D$ is symmetric and positive definite and $C$ and $E$ are diagonal and such that $C^{2}+E^{2} \neq 0$, see [57]). This general model applies to most reaction-diffusion problems in the literature.

\section{(b) $2 D$ binary comection in a slender container}

In the usual Boussinesq approximation the governing equations are

$$
\begin{aligned}
& \nabla \cdot \boldsymbol{v}=0 \\
& \boldsymbol{v}_{t}+(\boldsymbol{v} \cdot \nabla) \boldsymbol{v}=\sigma \Delta \boldsymbol{v}-\nabla p+\sigma(\theta-c) e_{2} \\
& \theta_{t}+(\boldsymbol{v} \cdot \nabla) \theta=\Delta \theta+R v_{2} \\
& c_{t}+(\boldsymbol{v} \cdot \nabla) c=\lambda \Delta c+s \lambda \Delta \theta-s R v_{2} \\
& \boldsymbol{v}=\mathbf{0} \quad \theta=c_{y}=0 \quad \text { at } y=0,1 \\
& \boldsymbol{v}=\mathbf{0} \quad c_{x}=\theta \pm \gamma \theta_{x}=0 \quad \text { at } x= \pm L / 2 .
\end{aligned}
$$

After convenient nondimensionalization of the space and time coordinates. $x, y$ and $t$, the velocity $\boldsymbol{v}=\left(v_{1}, v_{2}\right)$, the pressure $p$. the temperature deviation from the steady state profile $\theta$ and the concentration $c$, the problem depends on the Prandtl, Rayleigh and Lewis numbers, $\sigma, R$ and $\lambda$, the separation ratio $s$ and the thermal Biot number at the lateral wall. $\gamma$.

\section{(c) Restricted 3D pure Rayleigh-Benard convection}

With the same notation as above, the governing equations are

$$
\begin{aligned}
& \nabla \cdot v=0 \\
& \boldsymbol{v}_{t}+(\boldsymbol{v} \cdot \nabla) \boldsymbol{v}=\sigma \Delta v-\nabla p+\sigma \theta \boldsymbol{e}_{2} \\
& \theta_{t}+(\boldsymbol{v} \cdot \nabla) \theta=\Delta \theta+R v_{2} \\
& \boldsymbol{v}=\mathbf{0} \quad \theta=0 \quad \text { at } y=0,1 \\
& \boldsymbol{v}(x, y, z+\ell, t)=\boldsymbol{v}(x, y, z, t) \quad \theta(x, y, z+\ell, t)=\theta(x, y, z, t) \\
& \boldsymbol{v}=\mathbf{0} \quad \theta \pm \gamma \theta_{x}=0 \quad \text { at } x= \pm L / 2 .
\end{aligned}
$$

Here we are interested in the stability of rolls whose size in the $z$-direction is $\ell=O(1)$, while the slenderness of the container $L$ is large. We are considering a restricted problem because we do not allow $\ell$ to depend weakly on the $x$-coordinate.

In order to consider those problems (and many others) at the same time, we write the governing equations in an abstract form as

$D_{0} u_{t}=D_{1} u_{x x}+D_{2} u_{x}+\mathcal{L} u+\mathcal{B}\left(u, u_{x}\right)+f(u, \mu) \quad$ in $-L / 2<x<L / 2$

$C u \pm E u_{x}=0 \quad$ at $x= \pm L / 2$.

Here the unknown $u=u(x, t)$ is defined as a function of $[-L / 2, L / 2] \times[0, \infty[$ into a Hilbert space $S, D_{0}, D_{1}, D_{2}, C$ and $E$ are linear (not necessarily invertible) operators of $S$ into $S ; \mathcal{L}: \mathcal{D}(\mathcal{L}) \rightarrow S$ is a linear operator with domain $\mathcal{D}(\mathcal{L}) \subset S, \mathcal{B}: S \times S \rightarrow S$ is a bilinear operator and $f: \mathcal{D}_{1} \times \mathbb{R} \rightarrow S$ is a nonlinear operator, with domain $\mathcal{D}_{1} \times \mathbb{R} \subset S \times \mathbb{R}$. The length $L$ and the control parameter $\mu$ are such that $L \gg 1$ and $|\mu| \ll 1$.

The problems (a)-(c) above may be written in the form (2.5), (2.6). To this end, the variable $u$, the space $S$, the operators $\mathcal{L}$ and $\mathcal{B}$ and the domains $\mathcal{D}(\mathcal{L})$ and $\mathcal{D}_{1}$ may be defined, for example. as 
(a) $S=\left[L_{2}(\Omega)\right]^{N}, \mathcal{L}(u)=D u_{y y}$ in $2 \mathrm{D}$ or $\mathcal{L}(u)=D\left(u_{y y}+u_{z z}\right)$ in $3 \mathrm{D}$

$\mathcal{B}=0, \mathcal{D}(\mathcal{L})=\left\{u \in\left(W_{2}^{2}(\Omega)\right)^{N}: u\right.$ satisfies (A.1) $\}$

(b) $u=\left(p, v_{1}, v_{2}, \theta, c\right), S=\left(L_{2}(] 0,1[j)^{5}, \mathcal{D}_{1}=\left(W_{2}^{1}(] 0,10\right)\right)^{5}$,

$\mathcal{D}(\mathcal{L})=\left\{u \in W_{2}^{1}(] 0,1[) \times\left[W_{2}^{2}(] 0,1[)\right]^{4}: u\right.$ satisfies (A.2) $\}$

$\mathcal{L}(u)=\left(v_{2 y}, \sigma v_{1 y y}, \sigma v_{2 y y}-p_{y}, \theta_{y y}, \lambda c_{y y}+s \lambda \theta_{y y}\right)$

$\mathcal{B}\left(u, u_{x}\right)=-\left\langle 0, v_{1} v_{1 x}, v_{1} v_{2 x}, v_{1} \theta_{x}, v_{1} c_{x}\right)$

$f(u, \mu) \equiv\left(0,-v_{2} v_{1 y},-v_{2} v_{2 y}+\sigma(\theta-c),-v_{2} \theta_{y}+R v_{2},-v_{2} c_{y}-s R v_{2}\right)$

(c) $u=\left(p, v_{1}, v_{2}, v_{3}, \theta\right), S=\left[\left(L_{2}(] 0,1[\times] 0, L[j]^{5}, \mathcal{D}_{1}=\left[W_{2}^{1}(] 0,1[\times] 0, \ell[\mathrm{j}]^{5}\right.\right.\right.$

$\mathcal{D}(\mathcal{L})=\left\{u \in W_{2}^{1}(] 0,1[\times] 0, \ell[) \times\left[W_{2}^{2}(] 0,1[\times] 0, \ell[)^{4}: u\right.\right.$ satisfies (A.3) and (A.4) $\}$

$\mathcal{L}(u)=\left(v_{2 y}+v_{3 z}, \sigma\left(v_{1 y y}+v_{1 z z}\right), \sigma\left(v_{2 y y}+v_{2 z z}\right)-p_{y}, \sigma\left(v_{3 y y}+v_{3 z z}\right)-p_{z}, \theta_{y y}+\theta_{z z}\right)$

$\mathcal{B}\left(u, u_{x}\right)=-\left(0, v_{1} u_{1 x}, v_{1} v_{2 x}, v_{1} v_{3 x}, v_{1} \theta_{x}\right)$

$f(u, \mu)=\left(0,-v_{2} v_{1 y}-v_{3} v_{1 z},-v_{2} v_{2 y}-v_{3} v_{2 z}+\sigma \theta,-v_{2} v_{3 y}-v_{3} v_{3 z},-v_{2} \theta_{y}-v_{3} \theta_{z}+R v_{2}\right)$

while the remaining operators. $D_{0}, D_{1}, D_{2}, C$ and $E$, are obviously defined in each case. Here. $L_{2}(\Omega)$ is the space of those (classes of) functions whose square is integrable in $\Omega$, with the usual inner product, and. for $p=\mathrm{I}$ and $2 . W_{2}^{p}$ is the usual Sobolev space of those (classes of) functions whose derivatives. up to order $p$, belong to $L_{2}(\Omega)$; then the boundary conditions (A.1)-(A.4) in the definitions of $\mathcal{D}(\mathcal{L})$ must be assumed to be satisfied in the weak sense. The choice of the space $S$ above is not essential in the formal analysis in this appendix; it is necessary only to define an inner product. Also, the abstract formulation above is appropriate here but it would not be the appropriate one if we were interested in the well-posedness (that is taken for granted in this paper) of problems (b) and (c) (see [58]).

Notice that (A.5) is invariant under $x$-translations. In addition we require (A.5) to be invariant under $x$-reflections. More precisely: there is a linear operator. $J: S \rightarrow S$, such that $J^{2}=$ identity, $J$ conmutes with $D_{0}, D_{1}, D_{2}$ and $\mathcal{L}$,

$$
\begin{aligned}
& \mathcal{B}(J u, J v)=J \mathcal{B}(u,-v) \quad \text { for all } u, v \in S \text { and } \\
& f(J u, \mu)=J f(u, \mu) \quad \text { for all } u \in \mathcal{D}_{1} \text { and all } \mu \in \mathbb{R}
\end{aligned} .
$$

Then (2.5) is invariant under the transformation $x \rightarrow-x, u \rightarrow J u$. Notice that this property is satisfied by the problems (a)-(c) above (with $J=$ identity for problem (a), $J\left(p,-v_{1}, v_{2}, \theta, c\right) \equiv\left(p, v_{1}, v_{2}, \theta, c\right)$ for problem (b) and $J\left(p, v_{1}, v_{2}, v_{3}, \theta\right)=$ $\left(p,-v_{1}, v_{2}, v_{3}, \theta\right)$ for problem (c)).

The abstract formulation (A.5), (A.6) also includes the mathematical models for TaylorConette flow and electrodynamic convection in nematic liquid crystals. mentioned in section $I$, and the usual hydrodynamic models in flame propagation [59], that are known to exhibit oscillatory instability [60]. Instead, (A.5), (A.6) do not include the free-boundary models for the experiments in capillary flows mentioned in section 1, but the analysis below may be extended straightforwardly to free-boundary problems.

The analysis below is a generalization of that in section 2 . We consider a steady state of (A.5), (A.6) that is independent of $x$ to a first approximation, except in two boundary layers, where $|x \pm L / 2| \sim 1$.

\section{A1. The solution of (A.5) in the bulk, $-L / 2<x<L / 2,|x \pm L / 2| \sim 1$}

Here the steady state is given by

$$
u=u_{0}+\mu u_{1}+\mu^{2} u_{2}+\cdots
$$

where $u_{0}, u_{1}$ and $u_{2}$ satisfy

$$
\mathcal{L} u_{0}+f_{0}=\mathcal{L} u_{1}+F_{1} u_{1}+f_{1}=0
$$




$$
\mathcal{L} u_{2}+F_{1} u_{2}+f_{2}+F_{2} u_{1}+\mathcal{B}_{1}\left(u_{1}, u_{1}\right)=0
$$

and the vectors $f_{0}, f_{1}$ and $f_{2}$ and the operators $F_{1}, F_{2}$ (linear) and $\mathcal{B}_{1}$ (bilinear) are defined in the Taylor expansion of $f$

$$
\begin{aligned}
f\left(u_{0}+U, \mu\right) & =f_{0}+\mu f_{1}+\mu^{2} f_{2}+\left(F_{1}+\mu F_{2}+\mu^{2} F_{3}\right) U+\mathcal{B}_{1}(U, U)+\mathcal{C}(U, U, U) \\
& +\mathrm{O}\left(|\mu|^{3}+|\mu|\|U\|^{2}+\|U\|^{4}\right) \quad \text { as }|\mu|+\|U\| \rightarrow 0 .
\end{aligned}
$$

Now we assume that the dispersion relation associated with the linear stability of the steady state (A.8) exhibits oscillatory instability at $\mu=0$. That is, if the ansatz $u=u_{0}+\mu u_{1}+\mu^{2} u_{2}+[U \exp (\Omega t+\mathrm{i} k x)+\mathrm{c} . \mathrm{c}$.$] is inserted into (A.5), the resulting$ linearized eigenvalue problem possesses two pairs of algebraically simple, complex conjugate eigenvalues satisfying (2.5). Now the real constant $b_{0}$ and the complex constants $b_{1}, c, d_{0}$ and $d_{1}$ are given by $(l,$,$\left.\rangle is the inner product of the Hilbert space S\right)$

$b_{0}=\left\langle\mathcal{L}_{1} U_{0}, U_{0}^{*}\right\rangle \quad c=\left\langle D_{1} U_{0}+\mathbf{i} b_{0} D_{0} U_{1}-\mathbf{i} \mathcal{L}_{1} U_{1}, U_{0}^{*}\right\rangle$

$d_{0}=\left\langle\mathcal{B}_{2}\left(u_{1}, U_{0}\right)+F_{2} U_{0}, U_{0}^{*}\right\rangle$

$b_{1}=\left\langle-b_{0} D_{0} U_{3}+\mathrm{i} d_{0} D_{0} U_{1}+\mathcal{L}_{1} U_{3}+\mathcal{B}\left(u_{1}, U_{0}\right)-\mathrm{i} F_{2} U_{1}-\mathrm{i} \mathcal{B}_{2}\left(u_{1}, U_{1}\right), U_{0}^{*}\right\rangle$

$d_{1}=\left\langle-d_{0} D_{0} U_{3}+F_{3} U_{0}+F_{2} U_{3}+\mathcal{B}_{2}\left(u_{1}, U_{3}\right)+\mathcal{B}_{2}\left(u_{2}, U_{0}\right)+3 \mathcal{C}\left(u_{1}, u_{1}, U_{0}\right), U_{0}^{*}\right\rangle$

and are obtained as solvability conditions of the following problems

$\mathcal{L}_{0} U_{1}=\mathbf{i} b_{0} D_{0} U_{0}-\mathrm{i} \mathcal{L}_{1} U_{0}$

$\mathcal{L}_{0} U_{2}=-c D_{0} U_{0}+D_{1} U_{0}+\mathrm{i} b_{0} D_{0} U_{1}-\mathbf{i} \mathcal{L}_{1} U_{1}$

$\mathcal{L}_{0} U_{3}=d_{0} D_{0} U_{0}-\mathcal{B}_{2}\left(u_{1}, U_{0}\right)-F_{2} U_{0}$

$\mathcal{L}_{0} U_{4}=\mathrm{i} b_{1} D_{0} U_{0}+\mathrm{i} b_{0} D_{0} U_{3}+d_{0} D_{0} U_{1}-\mathrm{i} \mathcal{L}_{1} U_{3}-\mathrm{i} \mathcal{B}\left(u_{1}, U_{0}\right)-F_{2} U_{1}-\mathcal{B}_{2}\left(u_{1}, U_{1}\right)$

$\mathcal{L}_{0} U_{5}=d_{1} D_{0} U_{0}+d_{0} D_{0} U_{3}-F_{3} U_{0}-F_{2} U_{3}-\mathcal{B}_{2}\left(u_{1}, U_{3}\right)-\mathcal{B}_{2}\left(u_{2}, U_{0}\right)-3 \mathcal{C}\left(u_{1}, u_{1}, U_{0}\right)$

where

$\mathcal{L}_{0} U \equiv\left(\mathcal{L}-\mathrm{i} \omega D_{0}-k_{0}^{2} D_{1}+\mathrm{i} k_{0} D_{2}+F_{1}\right) U+\mathrm{i} k_{0} \mathcal{B}\left(u_{0}, U\right)$

$\mathcal{L}_{1} U \equiv\left(2 \mathrm{i} k_{j} D_{1}+D_{2}\right) U+\mathcal{B}\left(u_{0}, U\right) \quad \mathcal{B}_{2}(u, U) \equiv \mathrm{i} k_{0} \mathcal{B}(u, U)+2 \mathcal{B}_{1}(u, U)$

$U_{0} \neq 0$ is any eigenvector of the linearized problem

$$
\mathcal{L}_{0} U_{0}=0
$$

and $U_{0}^{*}$ is a normalized eigenvector of the adjoint (with respect to the inner product $\langle$,$\rangle )$ linearized problem

$$
\mathcal{L}_{0}^{*} U_{0}^{*}=0 \quad\left\langle D_{0} U_{0}, U_{0}^{*}\right\rangle=1 .
$$

Notice that, as in section 2.1, the constants $b_{0}, b_{1}, c, d_{0}$ and $d_{1}$ do not depend on the particular solutions of (A.16), (A.18) and (A.23) that are selected, and that the normalizing condition in (A.24) makes sense because $\left\langle D_{0} U_{0}, U_{0}^{*}\right\rangle \neq 0$ whenever $U_{0} \neq 0$ and $U_{0}^{*} \neq 0$ satisfy (A.23) and the first equation in (A.24) (recall that the eigenvalue $i \omega$ of (A.23) is algebraically simple).

The weakly nonlinear evolution of $u$ is obtained. as in section 2.1. by considering the ansatz

$u=u_{0}+\mu u_{1}+\mu^{2} u_{2}+\left(A U_{\mathrm{j}} \mathrm{e}^{\mathrm{i} \omega t+1 k_{0} x}+B V_{\mathrm{j}} \mathrm{e}^{\mathrm{i} \omega t-k_{0}, x}+\right.$ c.c. $)+\cdots$ 
where the complex amplitudes are small and depend weakly on $x$ and $t$. The evolution equations for $A$ and $B$ are obtained, as in section 2.1 , to be given again by (2.16), (2.17), while $U$ is given by

$$
\begin{aligned}
u=u_{0}+\mu u_{1} & +\mu^{2} u_{2}+\left[\left(A U_{0}-\mathrm{i} A_{x}\left(U_{1}+\mu U_{4}\right)-A_{x x} U_{2}+\mu A\left(U_{3}+\mu U_{5}\right)\right.\right. \\
& \left.\left.+A|A|^{2} U_{8}+A|B|^{2} U_{9}+\text { HORT1 }\right) \mathrm{e}^{\mathrm{i} \omega t+i k_{0} x}+\mathrm{c} . \mathrm{c} .\right] \\
& +\left[\left(B V_{0}+\mathrm{i} B_{x}\left(V_{1}+\mu V_{4}\right)-B_{x x} V_{2}+\mu B\left(V_{3}+\mu V_{5}\right)+B|B|^{2} V_{8}\right.\right. \\
& \left.\left.+B|A|^{2} V_{9}+\text { HORT } 2\right) \mathrm{e}^{\mathrm{i} \omega t-1 k_{0} x}+\text { c.c. }\right] \\
& +\left[\left(A^{2} U_{6} \mathrm{e}^{i \mathrm{i} k_{0} x}+B^{2} V_{6} \mathrm{e}^{-2 i k_{0} x}\right) \mathrm{e}^{2 \mathrm{i} \omega t}+A B W_{1} \mathrm{e}^{2 \mathrm{i} \omega t}+A \bar{B} W_{2} \mathrm{e}^{3 i k_{0} x}+\text { c.c. }\right] \\
& +|A|^{2} U_{7}+|B|^{2} V_{7}+\text { HONRT }
\end{aligned}
$$

where HONRT, HORT1 and HORT2 are as in (2.19), (2.20) and, the vectors $U_{0}, \ldots, U_{5}$ are as obtained above, while the remaining vectors are given by

$\left(\mathcal{L}_{0}+\mathcal{L}_{22}\right) U_{6}=-\mathrm{i} k_{0} \mathcal{B}\left(U_{0}, U_{0}\right)-\mathcal{B}_{1}\left(U_{0}, U_{0}\right)$

$\left(\mathcal{L}_{0}+\mathcal{L}_{00}\right) U_{7}=\mathrm{i} k_{0} \mathcal{B}\left(U_{0}, \bar{U}_{j}\right)-\mathcal{B}_{2}\left(\bar{U}_{0}, U_{0}\right)$

$\left(\mathcal{L}_{0}+\mathcal{L}_{20}\right) W_{1}=\mathrm{i} k_{0} \mathcal{B}\left(U_{0}, V_{0}\right)-\mathcal{B}_{2}\left(V_{0}, U_{0}\right)$

$\left(\mathcal{L}_{0}+\mathcal{L}_{02}\right) W_{2}=-\mathrm{i} k_{0} \mathcal{B}\left(U_{0}, \bar{V}_{0}\right)-\mathrm{i} k_{0} \mathcal{B}\left(\bar{V}_{0}, U_{0}\right)-2 \mathcal{B}_{1}\left(\bar{V}_{0}, U_{0}\right)$

$\mathcal{L}_{0} U_{8}=e_{1} D_{0} U_{0}-\mathcal{B}_{2}\left(U_{7}, U_{0}\right)-\mathcal{B}_{3}\left(U_{6}, \bar{U}_{0}\right)-3 \mathcal{C}\left(U_{0}, U_{0}, \bar{U}_{0}\right)$

$\mathcal{L}_{0} U_{9}=e_{2} D_{0} U_{0}-\mathcal{B}_{2}\left(V_{7}, U_{\omega}\right)-\mathcal{B}_{2}\left(W_{1}, \bar{V}_{0}\right)-\mathcal{B}_{3}\left(W_{2}, V_{0}\right)-6 \mathcal{C}\left(U_{0}, V_{0}, \bar{V}_{0}\right)$

$V_{j}=J U_{j} \quad$ for $j=0, \ldots, 9$

where the linear operators $J, \mathcal{L}_{0}$ and $\mathcal{L}_{r s}$ for $r, s=0,2$, and the bilinear operators $\mathcal{B}_{2}$ and $\mathcal{B}_{3}$ are given by (A.6), (A.21), (A.22) and

$\mathcal{L}_{r s}=\left[\mathbf{i}(1-r) \omega D_{0}+\left(1-s^{2}\right) k_{i j}^{2} D_{1}+\mathrm{i}(s-1) k_{0} D_{2}\right] U+\mathrm{i}(s-1) k_{0} \mathcal{B}\left(u_{0}, U\right)$

$\mathcal{B}_{2}(U, V) \equiv \mathrm{i} k_{i} \mathcal{B}(U, V)+2 \mathcal{B}_{1}(U, V)$

$B_{3}(U, V) \equiv \mathcal{B}_{2}(U, V)+2 \mathbf{i} k_{i}[\mathcal{B}(V, U)-\mathcal{B}(U, V)]$.

As in section 2.1. $U_{6}, U_{7}, W_{1}$ and $W_{3}$ are uniquely determined by (A.26)-(A.29), while (A.30) and (A.3I) possess a solution if and only if

$e_{1}=\left\langle\mathcal{B}_{2}\left(U_{7}, U_{0}\right)+\mathcal{B}_{3}\left(U_{6}, \bar{U}_{0}\right)-3 \mathcal{C}\left(U_{0}, U_{0}, \bar{U}_{0}\right), U_{0}^{*}\right\rangle$

$e_{2}=\left\langle\mathcal{B}_{2}\left(V_{7}, U_{0}\right)+\mathcal{B}_{2}\left(W_{1}, \bar{V}_{0}\right)+\mathcal{B}_{3}\left(W_{2}, V_{0}\right)+6 \mathcal{C}\left(U_{0}, V_{0}, \bar{V}_{0}\right), U_{0}^{*}\right\rangle$

and this determines the coefficients $e_{1}$ and $e_{2}$ of (2.14), (2.15).

A2. The solution of (A.5), (A.6) in the boundary lavers, $|x \pm L / 2| \sim 1$

As in section 2.2 we consider only the boundary layer near $x=-L / 2$ and use the re-scaled coordinate

$$
\xi=x+L / 2 .
$$

The steady state in this boundary layer is

$$
u=u^{0}(\xi)+\mu u^{1}(\xi)+\cdots
$$

where $u^{1}$ and $u^{2}$ satisfy

$$
\begin{aligned}
& D_{1} u_{\xi \xi}^{0}+D_{2} u_{\xi}^{0}+\mathcal{L} u^{0}+\mathcal{B}\left(u^{0}, u_{\xi}^{0}\right)+f^{0}(\xi)=0 \\
& \mathcal{L}^{0} u^{1}+f^{1}(\xi)=0 \quad \text { in } 0<\xi<\infty \\
& C u^{0}-E u_{\xi}^{0}=C u^{1}-E u_{\xi}^{1}=0 \quad \text { at } \xi=0 \\
& u^{0} \rightarrow u_{0} \quad u^{1} \rightarrow u_{1} \quad \text { as } \xi \rightarrow \infty
\end{aligned}
$$


Here $u_{0}$ and $u_{1}$ are given by (A.9), (A.10), the linear operator $\mathcal{L}^{0}$ is given by

$$
\mathcal{L}^{0} u \equiv D_{1} u_{\xi \xi}+D_{2} u_{\xi}+\left[\mathcal{L}+F^{1}(\xi)\right] u+\mathcal{B}\left(u^{0}, u_{\xi}\right)+\mathcal{B}\left(u, u_{\xi}^{0}\right)
$$

and, for each $\xi$. the vectors $f^{0}$ and $f^{1}$ and the linear operator $F^{1}$ are defined in the Taylor expansion of $f$ around $u=u^{\circ}, \mu=0$,

$f\left(u^{0}(\xi)+U, \mu\right)=f^{0}+\mu f^{1}+\left(F^{1}+\mu F^{2}\right) U+\mathcal{B}^{1}(U, U)+\mathcal{C}^{1}(U, U, U)+\cdots$.

The multilinear operators $B^{1}$ and $\mathcal{C}^{1}$ will be used below and depend also on $\xi$. Notice that if (A.43) holds then

$$
\begin{array}{lccc}
f^{0} \rightarrow f_{0} & f^{1} \rightarrow f_{1} & F^{1} \rightarrow F_{1} & F^{2} \rightarrow F_{2} \\
\mathcal{B}^{1} \rightarrow \mathcal{B}_{1} & \text { and } \quad \mathcal{C}^{1} \rightarrow \mathcal{C} & \text { as } \xi \rightarrow \infty &
\end{array}
$$

where $f_{0}, f_{1}, F_{1}, F_{2} . \mathcal{B}_{1}$ and $\mathcal{C}$ are defined in the Taylor expansion (A.11).

We assume that (A.40), (A.42). (A.43) has a unique solution; this assumption must be analysed carefully in each particular case since it does not need to hold in semi-infinite multidimensional nonlinear problems, even for the simplest reaction-diffusion equations $[60,61]$. We also assume that the linearized problem

$$
\begin{aligned}
& \mathcal{L}^{0} U=\Omega D_{0} U \quad \text { in } 0<\xi<\infty \\
& C U-E U_{\xi}=0 \quad \text { at } \xi=0 \quad\|U\| \text { bounded as } \xi \rightarrow \infty
\end{aligned}
$$

has a nontrivial eigenfunction, $U^{0}$, associated with the eigenvalue $i \omega$, that satisfies

$$
\begin{aligned}
& \mathcal{L}^{0} U^{0}=\mathrm{i} \omega D_{0} U^{0} \quad \text { in } 0<\xi<\infty \\
& C U^{0}-E U_{\xi}^{0}=0 \quad \text { at } \xi=0 \quad\left\|U^{0}\right\| \text { bounded as } \xi \rightarrow \infty
\end{aligned}
$$

and that the remaining part of the spectnum has a negative real part and lies at a non-zero distance from any point of the imaginary axis different from $\pm i \omega$. Then $(\Omega=0$ does not belong to the spectrum of (A.47). (A.48) and) (A.41)-(A.43) uniquely determines $u^{1}$. Finally, we assume that the convergence in(A.43) is exponential, and that there is a complex constant $r_{1} \neq 0$ such that the eigenfunctions of (A.49), (A.50) satisfy

$\left\|U^{0}(\xi)-U_{0} \mathrm{e}^{i k_{0} \xi}-r_{1} V_{0} \mathrm{e}^{-i k_{0} \xi}\right\| \rightarrow 0 \quad$ exponentially as $\xi \rightarrow \infty$.

These are again nontrivial issues for semi-infinite multidimensional problems, specially in the nonlinear case $[63,64]$. Fortunately, the assumptions made above about the spectrum of (A.47), (A.48) imply that the convergence (A.43) is exponential; this may be proven by applying classical general results in [65,66] for the linear case, and those in [67] for the nonlinear case. The assumption (A.51) instead must be analysed in each particular case. Notice that our assumptions imply that the convergence in (A.46) is also exponential.

Under the assumptions above, the weakly nonlinear evolution of the solutions of (A.5), (A.6) in this boundary layer is given by

$$
\begin{aligned}
u=u^{0}+\mu u^{1} & +\left[\left(a U^{0}+a \mu U^{1}+a|a|^{2} U^{4}+a_{t} U^{5}+\text { HORT }\right) \mathrm{e}^{\mathrm{i} \omega t}+a^{2} U^{2} \mathrm{e}^{2 i \omega t}+\text { c.c. }\right] \\
& +|a|^{2} U^{3}+\text { HONRT }
\end{aligned}
$$

where HORT and HONRT are as in (2.34). the complex amplitude $a$ is small and depends weakly on time. $U^{0} \neq 0$ is given by (A.49), (A.50) and $U^{1}, \ldots, U^{5}$ are now given by

$$
\begin{aligned}
& \mathcal{L}^{0} U^{1}-\mathrm{i} \omega D_{0} U^{1}=-F^{2} U^{0}-2 \mathcal{B}^{2}\left(u^{1}, U^{0}\right) \\
& \mathcal{L}^{0} U^{2}-2 \mathrm{i} \omega D_{0} U^{2}=-\mathcal{B}^{2}\left(U^{0}, U^{0}\right) \\
& \mathcal{L}^{0} U^{3}=-2 \mathcal{B}^{2}\left(U^{0}, \bar{U}^{0}\right) \\
& \mathcal{L}^{0} U^{4}-i \omega D_{0} U^{4}=-2 \mathcal{B}^{2}\left(\bar{U}^{0}, U^{2}\right)-2 \mathcal{B}^{2}\left(U^{0}, U^{3}\right)-3 \mathcal{C}^{1}\left(U^{0}, U^{0}, \bar{U}^{0}\right)
\end{aligned}
$$


$\mathcal{L}^{0} U^{5}-i \omega D_{0} U^{5}=D_{0} U^{0} \quad$ in $0<\xi<\infty$

$C U^{j}-E U_{\xi}^{j}=0 \quad$ at $\xi=0$

$U^{j}$ diverges at most algebraically as $\xi \rightarrow \infty$

for $j=1, \ldots, 5$. The bilinear operator $\mathcal{B}^{2}$ is given by

$$
\mathcal{B}^{2}(U, V)=-2 \mathcal{B}^{1}(U, V)-\mathcal{B}\left(U, V_{x}\right)-\mathcal{B}\left(V, U_{x}\right)
$$

and the operators $F^{2}$ and $\mathcal{C}^{1}$ are defined in (A.45). For matching purposes we only need the asymptotic behaviour of $U^{1}, \ldots, U^{5}$ at $\xi=\infty$. When taking into account that the convergence is exponential in (A.43), (A.46) and (A.51), it is seen that

$$
\begin{aligned}
U^{1}= & {\left[b_{0}^{-1} d_{0}\left(-\xi U_{0}+\mathrm{i} U_{1}\right)+U_{3}\right] \mathrm{e}^{\mathrm{i} k_{0} \xi}+r_{1}\left[b_{0}^{-1} d_{0}\left(\xi V_{0}+\mathrm{i} V_{1}\right)+V_{3}\right] \mathrm{e}^{-\mathrm{i} k_{0} \xi}+\mathrm{EST} } \\
U^{2}= & U_{6} \mathrm{e}^{2 i k_{0} \xi}+r_{1}^{2} V_{6} \mathrm{e}^{-2 i k_{0} \xi}+r_{1} W_{1}+\mathrm{EST} \\
U^{3}= & \bar{r}_{1} W_{2} \mathrm{e}^{2 \mathrm{i} \mathrm{k}_{0} \xi}+\text { c.c. }+U_{7}+\left|r_{1}^{2}\right| V_{7}+\mathrm{EST} \\
U^{4}= & {\left[b_{0}^{-1}\left(e_{1}+\left|r_{1}\right|^{2} e_{2}\right)\left(-\xi U_{0}+\mathrm{i} U_{1}\right)+U_{8}+\left|r_{1}\right|^{2} U_{9}\right] \mathrm{e}^{\mathrm{i} k_{0} \xi} } \\
& \quad+r_{1}\left[b_{0}^{-1}\left(e_{1}\left|r_{1}\right|^{2}+e_{2}\right)\left(\xi V_{0}+\mathrm{i} V_{1}\right)+\left|r_{1}\right|^{2} V_{8}+V_{9}\right] \mathrm{e}^{-\mathrm{i} k_{0} \xi}+\mathrm{EST} \\
U^{5}= & b_{0}^{-1}\left(\xi U_{0}-\mathrm{i} U_{1}\right) \mathrm{e}^{\mathrm{i} k_{0} \xi}+r_{1} b_{0}^{-1}\left(-\xi V_{0}-\mathrm{i} V_{1}\right) \mathrm{e}^{-i k_{0} \xi}+\mathrm{EST}
\end{aligned}
$$

where the vectors $U_{j}, V_{j}$ (for $j=0, \ldots, 9$ ). $W_{1}$ and $W_{2}$ are solutions of (A.16)-(A.20), (A.23) and (A.26)-(A.32), the constants $b_{0} . c, d_{0}, e_{1}$ and $e_{2}$ are given by (A.12). (A.13) and (A.36), (A.37), and EST stand for exponentially small terms as $\xi \rightarrow \infty$.

\section{A3. Matching between the solutions in the bulk and in the boundary lavers}

As in section 2.3 we apply matching conditions between the solutions in the bulk and in the boundary layer at $x=-L / 2$, to obtain the boundary conditions to be applied to the solutions in the bulk. To this end we write the solutions in the bulk and in the boundary layer in terms of the variable (A.38) and consider the limit

$$
1 \ll \xi \ll \lambda
$$

where $\lambda$ is as in section 2.3. The solution in the bulk is obtained by replacing (A.38) into (A.25') to obtain

$$
\begin{aligned}
& u=\left[\left(A_{0}+A_{0 x} \xi\right) U_{0}-\mathrm{i} A_{0, x} U_{1}-A_{0, x x} U_{2}+\mu A_{0} U_{3}\right. \\
& +A_{0}\left(\left|A_{0}\right|^{2} U_{8}+\left|B_{0}\right|^{2} U_{9}\right)+\text { HORT1 } \mathrm{e}^{\mathrm{i} \omega t+i k_{0} \xi-i k_{0} L / 2} \\
& +\left[\left(B_{0}+B_{0 x} \xi\right) V_{0}+\mathrm{i} B_{0 x} V_{1}-B_{0 x x} V_{2}+\mu B_{0} V_{3}\right. \\
& +B_{0}\left(\left|B_{0}\right|^{2} V_{8}+\left|A_{0}\right|^{2} V_{9}\right)+\text { HORT2] } \mathrm{e}^{\mathrm{i} \omega t-1 k_{0} \xi+1 k_{0} L / 2}+\text { c.c. + NRT }
\end{aligned}
$$

and the solution in the boundary layer is obtained by placing (A.51) and (A.60)-(A.64) into (A.52), to give

$$
\begin{aligned}
u=\left[a U_{0}+b_{0}^{-1}\right. & \left(a_{t}-d_{0} \mu a-\left(e_{1}+\left|r_{1}\right|^{2} e_{2}\right) a|a|^{2}\right)\left(\xi U_{0}-\mathrm{i} U_{1}\right)+a \mu U_{3} \\
& \left.+a|a|^{2}\left(U_{8}+\left|r_{1}\right|^{2} U_{9}\right)+\text { HORT }\right] \mathrm{e}^{\mathrm{l} \omega t+\mathrm{i} k_{0} \xi} \\
& +r_{1}\left[a V_{0}+b_{0}^{-1}\left(-a_{t}+d_{0} \mu a+\left(e_{1}+\left|r_{1}\right|^{2} e_{2}\right) a|a|^{2}\right)\left(\xi V_{0}+\mathrm{i} V_{1}\right)+a \mu V_{3}\right. \\
& +a|a|^{2}\left(\left|r_{1}\right|^{2} V_{8}+V_{9}\right)+\text { HORT } \mathrm{e}^{\mathrm{i} \omega t-\mathrm{i} k_{0} \xi}
\end{aligned}
$$

where HORT1, HORT2, HORT and NRT, $A_{0}, B_{0}, A_{0 x}$ and $B_{0 . x}$ are as in (2.56), (2.57). Then, by identifying (A.66) and (A.67) we obtain (2.58), (2.59) again. As a consequence, (2.61) holds and the derivation of the boundary conditions at $x=-L / 2$ is complete. The boundary conditions (2.63) are obtained in a completely similar way. 
Here we obtain sufficient conditions for properties 1 and 2 in section 5.1 to hold; more general conditions are omitted for the sake of brevity and will be considered elsewhere [44]. To proceed we first apply a reflection principle at $\xi= \pm I / 2$ to rewrite (5.27)-(5.31) as

$U_{k T}-U_{k \xi}=-\left(k^{2}+2 f\right) U_{k}+\alpha_{2} k^{2} V_{k}$

$V_{k T}-V_{k \xi}=-\left(\alpha_{2} k^{2}+2 \alpha_{3} f\right) U_{k}-k^{2} V_{k}$

$U_{k}(\xi+2, T)=U_{k}(\xi, T) \quad V_{k}(\xi+2, T)=V_{k}(\xi, T) \quad$ in $-\infty<\xi<\infty$

$U_{k}(\xi, 0)=U_{k 0}(\xi) \quad V_{k}(\xi, 0)=V_{k u}(\xi)$

where

$$
\begin{aligned}
& U_{k}(\xi, T)=u_{k}^{+}(\xi, T) \\
& V_{k}(\xi, T)=v_{k}^{+}(\xi, T) \\
& f(\xi, T)=\left|Y_{0}(\xi, T)\right|^{2} \quad \text { if }-1 / 2 \leqslant \xi<1 / 2 \\
& U_{k}(\xi, T)=u_{k}^{-}(-1-\xi, T) \\
& V_{k}(\xi, T)=v_{k}^{-}(-1-\xi, T) \\
& f(\xi, T)=\left|Z_{j}(-1-\xi, T)\right|^{2} \quad \text { if }-3 / 2 \leqslant \xi<-1 / 2 \\
& f(\xi+2, T)=f(\xi, T) \quad \text { if }-\infty<\xi<\infty .
\end{aligned}
$$

Equations (5.10)-(5.12) and (B.5)-(B.7) imply that if $\rho=1$ then the function $f$ is smooth in $-\infty<\xi<\infty, 0 \leqslant T<\infty$, while if $\rho \neq 1$ then $f$ is only piecewise smooth (it is discontinuous at $\xi=1 / 2+m$ for each integer $m$ and each $T \geqslant 0$ ); then some care must be taken below when obtaining general properties concerning the asymptotic behaviour of $U_{k}$ and $V_{k}$ as $T \rightarrow \infty$. Also, as was seen in section 5.2, in the supercritical case, $\alpha_{1}>-1$, $\left|Y_{0}\right|$ and $\left|Z_{0}\right|$ are uniformly bounded above in $-1 / 2 \leqslant \xi \leqslant 1 / 2$ for all $T>0$. In some cases (e.g. when analysing solutions of $(5.10)-(5.12)$ that converge to either a steady state or a limit cycle as $T \rightarrow \infty)$. $\left|Y_{0}\right|$ and $\left|Z_{0}\right|$ are also uniformly bounded below by a strictly positive constant as $T \rightarrow \infty$. Then we shall assume that $f>0$ is uniformly bounded above in $-\infty<\xi<\infty$ for all $T>0$ : also. we shall consider the case when $f$ is uniformly bounded below by a strictly positive constant. in $-\infty<\xi<\infty$, for all $T>0$.

Notice that when using the characteristic variable

$$
\eta=T-\xi
$$

equations (B.1)-(B.4) are reduced to the following pair of ODEs:

$$
\begin{aligned}
& U_{k \eta}=-\left(k^{2}+2 \tilde{f}\right) U_{k}+\alpha_{2} k^{2} V_{k} \\
& V_{k \eta}=-\left(\alpha_{2} k^{2}+2 \alpha_{3} \tilde{f}\right) U_{k}-k^{2} V_{k} \\
& U_{k}(\xi,-\xi)=U_{k 0}(\xi) \quad V_{k}(\xi,-\xi)=V_{k 0}(\xi)
\end{aligned}
$$

where the variable $\xi$ acts as a parameter and

$$
\tilde{f}(\xi, \eta) \equiv f(\xi, \xi+\eta) \text {. }
$$

Notice also that since $U_{k}$ and $V_{k}$ satisfy the periodicity conditions (B.3), in order to obtain asymptotic bounds as $T \rightarrow \infty$, that are uniformly valid in $-\infty<\xi<\infty$, we only need to obtain bounds valid in one period, e.g. in $0<\xi \leqslant 2$.

If $\left|Y_{0}\right|$ and $\left|Z_{0}\right|$ are constant (this requires the boundaries to be perfectly reflecting. i.e. $\rho$ to be equal to $\mathrm{I}$. see (5.10)-(5.12)). then the function $\bar{f}$ is constant (see (B.5), (B.6)) and 
the solutions of (B.9), (B.10) are readily obtained in closed form. Then the following result applies.

Lemma B.1. Let $\tilde{f}>0$ be a constant. If $\mathbf{I}+\alpha_{2} \alpha_{3}>0$ then every solution of $(B .9)$. (B.10) satisfies

$$
\left|U_{k}\right|+\left|V_{k}\right| \leqslant K_{0} \exp \left[-\lambda_{1}(k) \eta\right]
$$

where $K_{0}$ is a constant depending on the initial conditions, $\lambda_{1}(k)=\left(k^{2}+\tilde{f}\right)$ if $\mu(k) \equiv$ $\tilde{f}^{2}-2 \alpha_{2} \alpha_{3} k^{2} \tilde{f}-\alpha_{2}^{2} k^{4}<0$ and $\lambda_{1}(k)=\left[2\left(\mathbf{I}+\alpha_{2} \alpha_{3}\right) \tilde{f}+\left(\mathbf{I}+\alpha_{2}^{2}\right) k^{2}\right] k^{2} /\left(k^{2}+\tilde{f}+\sqrt{\mu(k)}\right)$ otherwise. If $\mathbf{I}+\alpha_{2} \alpha_{3}<0$ and $\mu(k)>0$ then there is a solution of (B.9). (B.I0) such that

$$
\left|U_{k}\right|+\left|V_{k}\right| \geqslant K_{0} \exp \left[\lambda_{2}(k) \eta\right]
$$

where $\lambda_{2}(k)=\left[2\left(1+\alpha_{2} \alpha_{3}\right) \tilde{f}+\left(1+\alpha_{2}^{2}\right) k^{2}\right] k^{2} /\left(\left(k^{2}+\tilde{f}\right)-\sqrt{\mu(k)}\right)>0$.

Proof. The result follows when solving (B.9), (B.10) in closed-form.

Lemma B.2. Let $f$ be a piecewise smooth, positive function, and let the constants $\alpha_{2}$ and $\alpha_{3}$ be such that $-1<\alpha_{2} \alpha_{3}<1$. Then, every solution of $(B .1)-(B .4)$ satisfies

$$
\left|U_{k}\right|+\left|V_{k}\right| \leqslant K_{0} \exp \left(-\lambda k^{2} T\right) \quad \text { as } T \rightarrow \infty
$$

for all $\xi$, where the constant $K_{0}$ depends on the initial conditions (B.4) and

$\lambda=1+\alpha_{2} \alpha_{3}$ if $-1<\alpha_{2} \alpha_{3} \leqslant 0 \quad \lambda=1-\alpha_{2} \alpha_{3} \quad$ if $0<\alpha_{2} \alpha_{3}<1$.

Proof. If $\alpha_{2}=0$ then the result readily follows from (B.9), (B.10).

If $\alpha_{2} \neq 0$ then we introduce the new dependent variable

$$
W_{k}=\alpha_{2}\left(\alpha_{3} U_{k}-V_{k}\right) \exp \left[\left(\mathbf{I}+\alpha_{2} \alpha_{3}\right) k^{2} \eta\right]
$$

to reduce (B.9)-(B.11) to

$$
\begin{aligned}
& W_{k \eta \eta}+2\left(\tilde{f}-\alpha_{2} \alpha_{3} k^{2}\right) W_{k \eta}+\alpha_{2}^{2}\left(1+\alpha_{3}^{2}\right) k^{4} W_{k}=0 \\
& W_{k}(\xi,-\xi)=\alpha_{2}\left[\alpha_{3} U_{k 0}(\xi)-V_{k 0}(\xi)\right] \exp \left[-\left(1+\alpha_{2} \alpha_{3}\right) k^{2} \xi\right] \\
& W_{k \eta}(\xi,-\xi)=\alpha_{2}^{2}\left(1+\alpha_{3}^{2}\right) k^{2} U_{k 0}(\xi) \exp \left[-\left(1+\alpha_{2} \alpha_{3}\right) k^{2} \xi\right] .
\end{aligned}
$$

Notice that

$\alpha_{2}^{2}\left(1+\alpha_{3}^{2}\right) k^{2} U_{k}=W_{k \eta} \exp \left[-\left(1+\alpha_{2} \alpha_{3}\right) k^{2} \eta\right]$

$\alpha_{2}^{2}\left(1+\alpha_{3}^{2}\right) k^{2} V_{k}=\left[\alpha_{3} W_{k \eta}-\alpha_{2}\left(\mathbf{I}+\alpha_{3}^{2}\right) k^{2} W_{k}\right] \exp \left[-\left(\mathbf{I}+\alpha_{2} \alpha_{3}\right) k^{2} \eta\right]$.

Now, when multiplying (B.13) by $W_{k \eta}$ and integrating in ] $-\xi, \eta[$ we obtain

$$
W_{k \eta}^{2}+\alpha_{2}^{2}\left(1+\alpha_{3}^{2}\right) k^{4} W_{k}^{2} \leqslant K_{1}+4 \int_{-\xi}^{\eta}\left(\alpha_{2} \alpha_{3} k^{2}-\tilde{f}\right) W_{k \eta}^{2} \mathrm{~d} \eta
$$

if $0 \leqslant \xi \leqslant 2$ and $\eta \geqslant-\xi$, where

$$
K_{1}=\alpha_{2}^{4}\left(1+\alpha_{3}^{2}\right) k^{4} \max _{0 \leqslant \xi \leqslant 2}\left\{\left(1+\alpha_{3}^{2}\right) U_{k 0}(\xi)^{2}+\left[\alpha_{3} U_{k 0}(\xi)-V_{k 0}(\xi)\right]^{2}\right\} .
$$

If $-1<\alpha_{2} \alpha_{3} \leqslant 0$ then $W_{k \eta n}^{2}+\alpha_{2}^{2}\left(1+\alpha_{3}^{2}\right) k^{4} W_{k}^{2} \leqslant K_{1}$ (see (B.18)) and the result readily follows when taking into account (B.16), (B.17). If $0<\alpha_{2} \alpha_{3}<1$ then by applying Gronwall's lemma to (B.18) we obtain

$$
W_{k \eta}^{2} \leqslant K_{1} \exp \left[4 \alpha_{2} \alpha_{3} k^{2}(\eta+\xi)\right] \leqslant K_{1} \exp \left[4 \alpha_{2} \alpha_{3} k^{2}(\eta+2)\right]
$$

if $0<\xi \leqslant 2$ and $\eta \geqslant-\xi$; when taking into account (B.16). (B.17) the result follows again, and the proof is complete. 
Lemma B.3. Let $f$ be uniformly bounded above and satisfy the assumptions in lemma B.2, and let the constant $F^{*}$ be given by

$$
F^{*}=\sup _{0 \leqslant \xi<2} \limsup _{\eta \rightarrow \infty} \frac{1}{\eta} \int_{-\xi}^{\eta} \tilde{f}(\xi, z) \mathrm{d} z=\sup _{0 \leqslant \xi \leqslant 2} \limsup _{T \rightarrow \infty} \frac{1}{T} \int_{0}^{T} f(\xi, z) \mathrm{d} z .
$$

In, addition

$$
\alpha_{2} \alpha_{3} \geqslant 1 \quad \text { and } k^{2}>\mu F^{*} \text { with } \mu=\sqrt{\alpha_{3}^{2}+1}-1 .
$$

then every solution of $(B .1)-(B .+)$ satisfies

$$
\left|U_{k}\right|+\left|V_{k}\right| \leqslant K_{i} \exp \left[\left(\mu F^{*}-k^{2}\right) T\right] \quad \text { as } T \rightarrow \infty
$$

for all $\xi$, where the constant $K_{0}$ depends on the initial conditions.

Proof. We introduce the new dependent variable

$$
W_{k}=U_{k} \exp \left(2 \tilde{F}+k^{2} \eta\right) \quad \text { with } \tilde{F}(\xi, \eta)=\int_{-\xi}^{\eta} \tilde{f}(\xi, z) \mathrm{d} z
$$

to write (B.9), (B. 10) as

$$
W_{k \eta \eta}-2 \tilde{f} W_{k \eta}+\left(\alpha_{2}^{2} k^{4}+2 \alpha_{2} \alpha_{3} \tilde{f} k^{2}\right) W_{k}=0 .
$$

Notice that $U_{k}$ and $V_{k}$ may be obtained in terms of $W_{k}$ and $W_{k \eta}$ by means of (B.19) and

$$
W_{k \eta}=\alpha_{2} k^{2} V_{k} \exp \left(2 \dot{F}+k^{2} \eta\right) \text {. }
$$

Now, we multiply (B.20) by $W_{k \eta} \exp (-4 \hat{F})$, integrate in $]-\xi, \eta[$ and integrate by parts to obtain

$$
\begin{gathered}
\left(W_{k \eta}^{2}+\alpha_{2}^{2} k^{4} W_{k}^{2}\right) \exp (-4 \tilde{F}) \leqslant K_{1}-4 \alpha_{2}^{2} k^{4} \int_{-\xi}^{\eta} \tilde{f}(\xi, z) W_{k}(\xi, z)^{2} \exp [-\mathbf{4} \tilde{F}(\xi, z)] \mathrm{d} z \\
-4 \alpha_{2} \alpha_{3} k^{2} \int_{-\xi}^{\eta} \tilde{f}(\xi, z) W_{k}(\xi, z) W_{k \eta}(\xi, z) \exp [-\mathbf{4} \tilde{F}(\xi, z)] \mathrm{d} z
\end{gathered}
$$

where the constant $K_{1}$ depends on the initial conditions. But, by Cauchy's inequality we have, for all $\lambda>0$,

$$
\begin{aligned}
& -\mathbf{4} \alpha_{2} \alpha_{3} k^{2} \int_{-\xi}^{\eta} \tilde{f}(\xi, z) W_{k}(\xi, z) W_{k \eta}(\xi, z) \exp [-\mathbf{4} \tilde{F}(\xi, z)] \mathrm{d} z \\
& \quad \leqslant 2 \alpha_{2} \alpha_{3} \int_{-\xi}^{\eta} \tilde{f}(\xi, z)\left[\lambda k^{4} W_{k}(\xi, z)^{2}+\lambda^{-1} W_{k \eta}(\xi, z)^{2}\right] \exp [-4 \tilde{F}(\xi, z)] \mathrm{d} z .
\end{aligned}
$$

If we select here

$$
\lambda=\left(\alpha_{2} / \alpha_{3}\right)\left(1+\sqrt{1+\alpha_{3}^{2}}\right)
$$

(the positive root of $\alpha_{3} \lambda^{2}-2 \alpha_{2} \lambda-\alpha_{2}^{2} \alpha_{3}=0$ ) and substitute in (B.22) we obtain

$$
\begin{gathered}
\left(W_{k \eta}^{2}+\alpha_{2}^{2} k^{4} W_{k}^{2}\right) \exp (-4 \tilde{F}) \leqslant K_{1}+\left(2 \alpha_{2} \alpha \alpha_{3} / \lambda\right) \int_{-\xi}^{\eta} \tilde{f}(\xi, z)\left[W_{k \eta}(\xi, z)^{2}+\alpha_{2}^{2} k^{4} W_{k}(\xi, z)^{2}\right] \\
\times \exp [-4 \tilde{F}(\xi, z)] \mathrm{d} z
\end{gathered}
$$

and, by applying Gronwall's inequality

$$
\exp (-4 \tilde{F})\left(W_{k \eta}^{2}+\alpha_{1} k^{4} W_{k}^{2}\right) \leqslant K_{2} \exp \left(2 \alpha_{2} \alpha_{3} \tilde{F} / \lambda\right)
$$

for a certain constant $K_{2}$ depending on initial conditions. When taking into account (B.19) and (B.2I) the result follows, and the proof is complete. 
Lemma B.4. Let $f$ be uniformly bounded below by a positive constant and satisfy the assumptions in lemma B.3, and let the constants $f_{*}$ and $f^{*}$ be given by

$$
\begin{aligned}
& f_{*}=\inf _{0 \leqslant \xi<2} \liminf _{\eta \rightarrow \infty} \tilde{f}(\xi, \eta)=\inf _{0 \leqslant \xi<2} \liminf _{T \rightarrow \approx} f(\xi, T) \\
& f^{*}=\sup _{0 \leqslant \xi 2} \limsup _{\eta \rightarrow \approx} \tilde{f}(\xi, \eta)=\sup _{0 \leqslant \xi<2} \limsup _{T \rightarrow \infty} f(\xi, T) .
\end{aligned}
$$

If $\alpha_{1} \alpha_{3}<-1$, then for each $k$ and each $\mu>0$ satisfying

$k^{2}<-2\left(1+\alpha_{1} \alpha_{3}\right) f_{*} /\left(1+\alpha_{2}^{2}\right)$

$\mu<-\left(k^{2}+f^{*}\right)+\sqrt{\left(k^{2}+f^{*}\right)^{2}-2\left(1+\alpha_{2} \alpha_{3}\right) k^{2} f_{*}-\left(\mathbf{I}+\alpha_{2}^{2}\right) k^{4}}$

there is a solution of (B.1)-(B.4) and a constant $T_{0}$ such that

$$
\left|U_{k}\right|+\left|V_{k}\right| \geqslant \exp (\mu T) \quad \text { if } T \geqslant T_{0} \text { and } \xi \in \mathbb{R} .
$$

Proof. Let the constants $f_{1}$ and $f_{2}$ be such that

$$
\begin{aligned}
& -\left(1+\alpha_{2}^{2}\right) k^{2} / 2\left(1+\alpha_{2} \alpha_{3}\right)<f_{1}<f_{*} \quad f^{*}<f_{2} \\
& \mu=-\left(k^{2}+f_{2}\right)+\sqrt{\left(k^{2}+f_{2}\right)^{2}-2\left(1+\alpha_{2} \alpha_{3}\right) f_{1}-\left(1+\alpha_{2}^{2}\right) k^{4}} .
\end{aligned}
$$

Such constants exist because $k$ and $\mu$ satisfy (B.23), (B.24) and the right-hand side of (B.26) decreases whenever (B.25) holds and either $f_{1}$ decreases or $f_{2}$ increases (and it vanishes as $\left.f_{1}=-\left(1+\alpha_{2}^{2}\right) k^{2} / 2\left(1+\alpha_{2} \alpha_{3}\right)\right)$. Also, (B.25) and the definition of $f_{*}$ and $f^{*}$ imply that there is a constant $\eta_{0}$ such that

$$
f_{1}<\tilde{f}(\xi, \eta)<f_{2} \quad \text { if } \eta \geqslant \eta_{0} \text { for all } \xi .
$$

Now, let us introduce the new variable

$$
W_{k}=\alpha_{2}\left(\alpha_{3} U_{k}-V_{k}\right) \exp (-\mu \eta)
$$

to write (B.9), (B. I0) as (see (B.26))

$W_{k \eta \eta}+2\left(\mu+k^{2}+\tilde{f}\right) W_{k \eta}+\left[2 \mu\left(\bar{f}-f_{2}\right)+2\left(1+\alpha_{2} \alpha_{3}\right) k^{2}\left(\tilde{f}-f_{1}\right)\right] W_{k}=0$

and, for each $\xi \in[-2,0]$, consider the solution of (B.29) such that

$$
W_{k}=\max \left\{\left|\alpha_{2} \alpha_{3}\right|,\left|\alpha_{2}\right|\right\} \quad W_{k \eta}=0 \quad \text { at } \eta=\eta_{0} .
$$

That solution increases as $\eta$ ( $\geqslant \eta_{0}$ ) increases because, since the coefficient of $W_{k}$ in (B.29) is negative (see (B.27)), $W_{k m}>0$ at $\eta=\eta_{0}$, and $W_{k}$ cannot have a local maximum if $\eta>\eta_{0}$. Then if $-2 \leqslant \xi \leqslant 0$ and $T \geqslant \eta_{0}+2$ we have (see (B.8) and (B.28))

$$
\left|U_{k}\right|+\left|V_{k}\right| \geqslant\left|W_{k}\right| / \max \left\{\left|\alpha_{2} \alpha_{3}\right|,\left|\alpha_{2}\right|\right\} \geqslant \exp (\mu \eta) \geqslant \exp (\mu T)
$$

and the result follows. Thus the proof is complete.

When collecting the results in lemmas B.1-B.4 and taking into account (B.5)-(B.8), the following result is obtained.

Theorem B.5. (a) If either (i) $-1<\alpha_{2} \alpha_{3}<1$, or (ii) $\alpha_{2} \alpha_{3} \geqslant 1$ and $k$ is not too small, or (iii) $\alpha_{2} \alpha_{3} \geqslant-1$ and both $\left|Y_{0}\right|$ and $\left|Z_{0}\right|$ are constant, then property 1 of section 5.1 applies.

(b) If $\alpha_{2} \alpha_{3}<-1$ and both $\left|Y_{0}\right|$ and $\left|Z_{0}\right|$ are uniformly bounded below by a strictly positive constant as $T \rightarrow \infty$, then property 2 of section 5.1 applies. 


\section{References}

[1] Cross M C and Hohenberg P C 1993 Pattern formation outside of equilibrium Rev. Mod. Phys. 65 $851-1112$

[2] Steinberg V, Fineberg J, Moses E and Rehberg I 1989 Pattern selection and transition to turbulence in propagating waves Physica 37D 359-83

[3] Bensimon D, Kolodner P. Surko C M, Williams H and Croquette V 1990 Competing and coexisting dyoanical states of travelling-wave convection in an annulus J. Fltuid Mech. $217441-67$

[4] Kolodner P 1993 Repeated transients of weakly nonlinear travelling-wave convection Phys. Rev. E 47 103848

[5] Kaplan E and Steinberg V 1993 Measurement of reflection of travelling waves near the onset of binary-fluid convection Phys. Rev. E 48 R661-4

[6] Predtechensky A A. McCormick W D, Swift J B, Noszticzius Z and Swinney H L 1994 Onset of travelling waves in isothermal double diffusive convection Phys. Rev. Lett. 72 218-21

[7] Motsay R W. Anderson K E and Behringer R P 1988 The onset of convection and turbulence in rectangular layers of normal liquid ${ }^{4} \mathrm{He} \mathrm{J}$. Flidid Aech. $189263-86$

[8] Croquette V and Willians $\mathrm{H} 1989$ Nonlinear competition between waves on convective rolls Phys. Rev, A $392765-8$

[9] Croquette V and Williams H 1989 Nonlinear waves of the oscillatory instability on finite convective rolls Physica 37D 300-14

[10] Andereck C D. Liu S S and Swinney H L 1986 Flow regimes in a circular Couette system with independently rotating cylinders $J$. Fiuid Mech. $164155-83$

[11] Tagg R, Edwards W S and Swinney H L 1990 Convective versus absolute instability in flow between counter-rotating cylinders Phys. Rev. A 42 831-7

[12] Dubois M. Daviaud F, Ronsin O and Bergé $\mathrm{P} 1992$ Traveling waves in pure fluids locally heated along wires Physica 61D 140-6

[13] Vince J M and Dubois M 1992 Hot wire below the free surface of a licuid: structural and dyoamical properties of a secondary instability Europhys. Lett. 20 505-10

[14] Daviaud F and Vince J M 1993 Travelling waves in a fluid layer subjected to a horizontal temperature gradient Phys. Rew. E 48 4432-6

[15] Joets A and Ribotta R 1988 Localized, time-dependent state in the convection of a nematic liquid crystal Phys. Rev. Lett. $602164-7$

[16] Rehberg I, Rasenat $S$ and Steinberg V 1989 Travelling waves and defect-initiated turbulence in electroconvecting nematics Phys. Rev, Lett. $62756-9$

[17] Reluberg I. Winkler B L, de la Torre M, Rasenat S and Schöpf W 1989 Pattern formation in a liquid crystal Advances in Solid State Physics vol 29. ed V Roessler (Braunschweig: Vieweg) pp 35-52

[18] de la Torre M 1990 Contribución al estudio de la formación de estructuras en fluidos anisótropos Doctoral Thesis Universidad Nacional de Educación a Distancia. Madrid

[19] Markstein G H 1964 Nonsteady Flame Propagation (New York: Plenum)

[20] Ferguson C R and Keck J C 1979 Stand-off distances on a that butner Combustion and Flame 34 85-98

[21] Sabathier F, Boyer L and Clavin P 1979 Experimental study of weak turbulent premixed flames Combustion and Flame 35 139-53

[22] Bucknaster J 1983 Stability of the porous plug burner tlame SLAM J. Appl. Math. 43 1335-49

[23] Margolis S B and Willians F A 1989 Diffusional-thermal instability of a solid propellant thame SIAA $J$. Appl. Math. $491390-420$

[24] Coullet $\mathrm{P}$, Fauve S and Tirapegui E 1985 Large scale instability of nonlinear standing waves J. Physique Lett. $46787-91$

[25] Hohenberg P C and Cross M C 1987 An introduction to pattern formation in non-equilibrium systens Lecture Notes in Physics vol 268 (Berlin: Springer)

[26] Fauve S 1987 Large scale instabilities of cellular flows Instabihties and Non-equilibmm Structures ed E Tirapegui and D Villarroel (Dordrecht: Reidel)

[27] Pierce R D and Knobloch E 1995 Asymptotically exact Zakharov equations and the stability of water waves with binodal spectra Physica 81D $341-73$

[28] Christodoulides P and Dias F 1994 Resonant capillary-gravity interfacial waves J. Flhd Mech. 265 303-43

[29] Nicolás J A, Martel C and Vega J M 1996 Weakly non-linear oscillations in liquid bridges under highfrequency axial forcing in preparation

[30] Knobloch E and Gibbon J D 1991 Coupled NLS equations for counter propagating waves in systems with reflection symmetry Phys. Lett. 154A 353-6

[31] Cross M C 1986 Travelling and standing waves in binary-fluid convection in finite geometries Phys. Rev. Lett. $\mathbf{5} 72935-8$ 
[32] Cross M C 1988 Structure of nonlinear travelling waves in finite geometries Phys, Rev, A 38 3593-600

[33] Cross M C 1989 Nonlinear travelling wave states in finite geonetries Physica 37D 315-8

[34] Cross M C and Kuo E Y 1992 One dimensional spatial structure near a Hopf bifurcation at finite wave number Physica 59D 90-120

[35] Bensimon D. Kolodner P, Surko C M. Williams H and Croquette V 1990 Competing and coexisting dynamical states of travelling-wave convection in an annulus $J$. Flatid Mech. 217 441-67

[36] Chikwendu S C and Kevorkian J 1972 A perturbation method for hyperbolic equations with small nonlinearities SIAM J. Appl. Math. 22 235-58

[37] Knobloch E and de Luca J 1990 Amplitude equations for travelling wave convection Nonlinearity $3575-80$

[38] Alvarez-Pereira C and Vega J M 1992 On the pulsating instability of two-dinensional tlames Eur. J. Appl. Nath. 3 55-73

[39] Vega J M 1993 On the amplitude equations arising at the onset of the oscillatory instability in pattern formation SIAM J. Math. Anal. 24 603-17

[40] Knobloch E 1992 Noulocal amplitude equations Pattern Formation in Complex Dissipative Systems ed S Kai (Singapore: World Scientific)

[41] Martel C 1995 La Inestabilidad Oscilatoria y sus Aplicaciones en Mecánica de Fluidos y Combustión Doctoral Thesis Universidad Politécnica de Madrid. Madrid

[42] Martel $\mathrm{C}$ and Vega J M 1996 A non-local amplitude equation that applies at the oscillatory instability in finite geometries, in preparation

[43] Martel C and Vega J M 1996 Global stability properties of a hyperbolic system arising in pattern formation Nonlinear Anal. TMA to appear

[44] Martel C and Vega J M 1996 Dynamics of a hyperbolic system that applies at the onset of the oscillatory instability, in preparation

[45] Knobloch E 1986 Oscillatory convection in binary mixtures Phys. Rev. A 34 1538-49

[46] Dangelmayr $\mathrm{G}$ and Knobloch $\mathrm{E} 1987$ The Physics of Structure Formation: Theory and Simulation ed $\mathrm{W}$ Güttinger and $G$ Dangelmayr (Berlin: Springer)

[47] Clever R M and Busse F H 1989 Nonlinear oscillatory convection in the presence of a vertical magnetic field $J$. Fltid wech. $201507-23$

[48] Schöpf W and Zimmermann W 1989 Multicritical behaviour in binary fluid convection Ekrophys. Lett. 8 41-6

[49] Dangelmayr G, Knobloch E and Wegelin M 1991 Dynamics of travelling waves in finite containers Europhys. Lett. 16 723-9

[50] Knobloch E and Silber M 1993 Oscillatory convection in a rotating layer Physica 63D 213-32

[51] Kevorkian J and Cole J D 1981 Perturbation Methods in Applied Mathematics (Berlin: Springer)

[52] Lagerstrom P A 1988 Matched Asymptotic Expansions (Berlin: Springer)

[53] Roberts A J 1992 Boundary conditions for approximate differential equations J. Austral. Afoth. Soc. Ser. B 34 54-80

[54] Jeffrey A and Kawalara T 1982 Asymptotic Methods in Noninear Wave Theory (Boston, MA: Pitman)

[55] Coddington E A and Levinson N 1955 Theory: of Ordinary Differential equations (New York: MoGraw-Hill)

[56] Gil L 1991 Space and time intermittency behaviour of a one-dinensional complex Ginzburg-Landau equation Nonlinearity $41213-22$

[57] Eidel'man S D 1969 Parabolic Systems (Amsterdam: North-Holland)

[58] Tenan R 1983 Novier-Stokes Equations and Nontinear Functional Anolysis (Society for Industrial and Applied Mathematics)

[59] Clavin P 1985 Dynamic behaviour of premixed flame fronts in laminar and turbulent flows Prog. Energy Combust. $S_{c r} 11$ 1-59

[60] Jackson T L and Kapila A K 1986 Thermal expansion effects on perturbed premixed flames Reacting Flows: Combustion and Chemical Reactors (Lectures in Applied Mathematics 24-I) ed G S S Ludford (Providence. RI: American Mathematical Society) pp 325-48

[61] Parra I E and Vega J M 1992 Multiple solutions of some semilinear elliptic equations in slender cylindrical domains J. Diff: Eqns $100225-56$

[62] Dancer E N 1993 Weakly nonlinear Dirichlet problens on long or thin domains Mfem. Am. Afath. Soc. 105 501

[63] Berestycki $\mathrm{H}$ and Nirenberg L 1990 Some qualitative properties of solutions of semilinear elliptic equations in eylindrical domains Analysis ... etc, Research Papers Published in Honor of Jitrgen Moser's 60th Birthday ed P H Rabinowitz and E Zelonder (New York: Acadenic) pp 115-64

[64] Vega J M 1993 The asymptotic beliaviour of the solutions of some semilinear elliptic equations in cylindrical domains $J$. Diff. Eqns 102 119-52 
[65] Agmon S and Nirenberg L 1963 Properties of solutions of ordinary differential equations in Banach space Commun. Pure Appl. Woth $16121-239$

[66] Pazy A 1967 Asymptotic expansion of solutions of ordinary differential equations in Hilbert space Arc. Rational Mech. Anal. 24 193-218

[67] Simon L 1985 Isolated singularity of extrema of geometric variational problems Harmonic Mappings and Minimal Immersions (Lecture Notes in Afathematics 1161) ed E Giusti (Berlin: Springer) 